\title{
Bifurcation to fully nonlinear synchronized structures in slowly varying media
}

\author{
Benoît Pier, Patrick Huerre, Jean-Marc Chomaz \\ Laboratoire d'Hydrodynamique (LadHyX) \\ CNRS - École polytechnique, F-91128 Palaiseau cedex, France
}

\begin{abstract}
The selection of fully nonlinear extended oscillating states is analysed in the context of one-dimensional nonlinear evolution equations with slowly spatially varying coefficients on a doubly-infinite domain. Two types of synchronized structures referred to as steep and soft global modes are shown to exist. Steep global modes are characterized by the presence of a sharp stationary front at the marginally absolutely unstable station and their frequency is determined by the corresponding linear absolute frequency, as in Dee-Langer propagating fronts. Soft global modes exhibit slowly varying amplitude and wavenumber over the entire domain and their frequency is determined by the application of a saddle point condition to the local nonlinear dispersion relation. The two selection criteria are compared and shown to be mutually exclusive. The onset of global instability first gives rise to a steep global mode via a saddle-node bifurcation as soon as local linear absolute instability is reached somewhere in the medium. As a result, such self-sustained structures may be observed while the medium is still linearly globally stable. Soft global modes only occur further above global onset and for sufficiently weak advection. The entire bifurcation scenario and state diagram are described in terms of three characteristic control parameters. The complete spatial structure of nonlinear global modes is analytically obtained in the framework of WKBJ approximations.
\end{abstract}

Keywords: Hydrodynamic stability; Frequency selection; Nonlinear global modes. PACS: 47.20.Ft; 47.20.Ky; 47.54.+r; 03.40.Kf.

\section{Introduction}

It is now well established that spatially developing open shear flows may be divided into two classes: some flows are very sensitive to inflow conditions and essentially behave as noise amplifiers, others display intrinsic dynamics and may be interpreted as global oscillators [20-22]. The present paper is concerned with the latter class of systems and examines in detail the synchronized self-sustained structures which they can support. In previous studies we 
have demonstrated the existence of nonlinear soft global modes [33] and steep global modes [34]. The objective of the present investigation is to analyse the bifurcation scenarii which lead from the basic state to either of these fully nonlinear structures as the global control parameters are varied. The analysis is carried out in the context of one dimensonal evolution models with spatially varying coefficients, in order to account for the streamwise development of the basic state.

A variety of physical systems give rise to intrinsic self-sustained oscillations: mixing layers with strong enough counterflow [45], low-density jets [43,29], cylinder wakes $[27,37,44]$, wakes behind blunt-edged plates $[18,19]$, thin aerofoilwakes [48], Taylor-Couette flow between concentric spheres [42], TaylorCouette flow between circular cylinders with throughflow [5], Rayleigh-Bénard convection with throughflow [31], baroclinically unstable atmospheric flows $[36,17]$, sunspot cycles $[1,28]$... Many of these flows display a spatially varying basic state, and hence a spatial dependence of the local instability characteristics. The goal of a global analysis is to obtain in a self-consistent manner a spatially extended structure made up of wave trains governed by the local properties of the medium and tuned at an overall global frequency $\omega_{g}$. The unknown global frequency $\omega_{g}$ is to be derived from a nonlinear eigenvalue problem consisting of the evolution equation and associated boundary conditions. The associated eigenfunction yields the spatial structure of the corresponding self-sustained oscillations. The resolution of the eigenvalue problem is typically undertaken in the context of a slowly varying approximation whereby the underlying basic state evolves slowly over a typical instability length scale. In this framework, the main objective of the global mode analysis is to derive global frequency selection criteria from the local dispersion relation prevailing at each streamwise station.

Linear global mode analyses are now fairly complete. Chomaz et al. [7] demonstrated that the complex global frequency is determined by a saddle point (equivalently a double turning point) condition applied to the local linear dispersion relation for the linear complex Ginzburg-Landau equation with spatially varying coefficients. This criterion had previously been discovered and implemented by Soward and Jones [42] to describe oscillating states in TaylorCouette flow between concentric spheres. According to Monkewitz et al. [30], the same criterion also holds for the Navier-Stokes equations linearized about an arbitrary slowly varying basic flow. More recently, Le Dizès et al. [25] reexamined the case of the spatially varying linear complex Ginzburg-Landau equation and demonstrated the existence of another family of linear global modes with two simple turning points. The causal nature of these linear global modes has been established for the same model by Hunt and Crighton [23]: the exact linear impulse response does converge, for large time, to the most unstable linear global mode. The validity of the linear saddle point criterion has been fully confirmed in the direct numerical simulations of the Kármán 
vortex street behind a blunt-edged plate by Hammond and Redekopp [19].

Paradoxically, the weakly nonlinear extension of these concepts is fraught with difficulties, as emphasized by Chomaz et al. [6] and Le Dizès et al. [24]: the Landau constant pertaining to the Hopf bifurcation near global mode onset does not display a well-defined sign as the WKBJ spatial inhomogeneity parameter is decreased. Furthermore, the weakly nonlinear formulation is only valid in an exponentially small vicinity of threshold.

To obviate such weakly nonlinear studies, it appears natural to resort to a fully nonlinear approach where fluctuations are of order unity. Such a line of thought has been consistently pursued since the early nineties in the framework of nonlinear Ginzburg-Landau type models. The classical absolute/convective instability concepts introduced in a linear context by Briggs [4] and Bers [3] have been generalized to the fully nonlinear régime by Chomaz [8]. The absolute/convective nature of the nonlinear dynamics is then directly related to the propagation direction of the front separating the basic state from the bifurcated state [16,38-40]. The properties of fully nonlinear global modes on a semi-infinite domain governed by Ginzburg-Landau type equations with constant coefficients have been thoroughly studied by Couairon and Chomaz $[10,12,13]$. In this case, a nonlinear global mode is obtained when an upstream travelling front is halted in its motion by the upstream boundary point. This event occurs whenever the medium is nonlinearly absolutely unstable in the sense of Chomaz [8]. The reader is referred to Tobias, Proctor and Knobloch [46] and Chomaz and Couairon [9] for a corresponding analysis of the finite-interval problem.

Corresponding fully nonlinear analyses have been performed for the complex Ginzburg-Landau equation with spatially varying coefficients in doubly infinite media. Two varieties of nonlinear global modes have been identified. Soft global modes, obtained by Pier and Huerre [33], obey a saddle point frequency selection criterion applied to the local nonlinear dispersion relation. This criterion is formally analogous to its linear counterpart. The associated spatial eigenfunction structure displays smoothly varying amplitude and wave number over the entire domain. By contrast, according to the preliminary results reported by Pier et al. [34], steep global modes are governed by a marginal linear instability criterion: the steep global frequency is imposed by the real absolute frequency [3] prevailing at the transition station between linear convective and absolute instability. This criterion is akin to the linear front velocity selection principle put forward by Dee and Langer [16]: for a wide class of systems, the speed of the front separating the basic state from the bifurcated state is such that in the co-moving frame the medium is marginally linearly absolutely unstable. The steep global spatial structure displays a stationary sharp front at the transition station with a sudden jump in wave number. In all other regions, the amplitude and wave number are slowly varying. Similar 
steep self-sustained structures have been numerically identified and analytically determined in amplitude evolution models pertaining to solar and stellar magnetic activity cycles by Meunier et al. [28] and Bassom et al. [1]. The properties of nonlinear global modes governed by the real Ginzburg-Landau equation in a semi-infinite domain with combined distributed spatial inhomogeneity have been obtained by Couairon [11] and Couairon and Chomaz [14]. Predicted scaling laws for amplitude and position of the maximum very favorably compare with experimental and numerical observations of bluff-body wakes.

The purpose of the present study is two-fold: first we wish to map out the domains of existence of soft or steep global modes in an appropriate control parameter space and to characterize the associated bifurcations. Secondly we present the detailed asymptotic structure of the various layers and regions which make up their spatial distribution.

Consider a system governed by a one-dimensional nonlinear partial differential equation that is first order in time of the form

$$
\frac{\partial \psi}{\partial t}=\mathcal{F}\left(\partial_{x} ; X\right)[\psi]
$$

where $x$ and $t$ represent space and time coordinates respectively, and $X$ is a slow space variable to be defined shortly. The basic state is assumed to be $\psi=$ 0 , and the function $\psi(x, t)$ represents the fluctuations riding on the basic state. In regions of finite amplitude, $\psi$ is governed by the full nonlinear operator $\mathcal{F}$. In small amplitude regions, $\psi$ is a perturbation governed by equation (1) linearized around the basic state, i.e.,

$$
\frac{\partial \psi}{\partial t}=\mathcal{L}\left(\partial_{x} ; X\right)[\psi]
$$

A crucial assumption of the present investigation is the slow spatial development of the medium as exemplified by the introduction of the slow spatial variable $X$ in the operators $\mathcal{F}$ and $\mathcal{L}$. The weak non-uniformity hypothesis is fulfilled if the ratio $\epsilon=\lambda / L$ between the typical instability length scale $\lambda$ and the inhomogeneity length scale $L$ is small. As a result of this scale separation, the weak variations of the medium instability properties may be described through the slow variable

$$
X=\epsilon x \quad \text { with } \quad \epsilon \ll 1
$$

If the slow space variable $X$ is frozen, system (1) becomes a p.d.e. in $x$ and $t$ with constant coefficients which captures the local instability properties prevailing at that station $X$. In order to construct a global mode it is necessary to "piece together" local wave trains at different $X$ by explicitly accounting for the weak coupling between local and global properties via relation (3). 
The outline of the paper is as follows. The bulk of the study (Sections 24) is concerned with the determination of the leading order approximation for fully nonlinear synchronized structures governed by (1). Local instability properties where $X$ is frozen are summarized in Section 2. Emphasis is given to the relationship between causality and the spatial response to a localized harmonic forcing (Section 2.3). In this framework, stationary fronts are shown to naturally arise as the limiting spatial response of the system in the absence of forcing when the medium is marginally absolutely unstable (Section 2.4). Variations of the local instability properties over the entire $X$-domain are analysed in Section 3. More specifically, the distribution over $X$ of linear spatial branches (Section 3.2) and nonlinear spatial branches (Section 3.3) is investigated as the global frequency is varied. Section 4 contains the essential results concerning the structure of steep global modes (Section 4.2), the nature of their bifurcation from the basic state (Sections 4.3-4.5), the structure of soft global modes (Section 4.6), and finally the respective domains of existence of steep and soft global modes (Sections 4.7,4.8) in control parameter space. The results of Sections $2-4$, in principle, apply to any nonlinear system governed by an equation of the form (1). In order to obtain explicit results, we repeatedly use as an illustrative example the complex Ginzburg Landau (CGL) equation

$$
\begin{aligned}
i \frac{\partial \psi}{\partial t}= & \left(\omega_{0}(X)+\frac{1}{2} \omega_{k k}(X) k_{0}(X)^{2}\right) \psi+i \omega_{k k}(X) k_{0}(X) \frac{\partial \psi}{\partial x} \\
& -\frac{1}{2} \omega_{k k}(X) \frac{\partial^{2} \psi}{\partial x^{2}}+\gamma(X)|\psi|^{2} \psi
\end{aligned}
$$

for a complex function $\psi(x, t)$. For convenience, the CGL equation is written here as derived from the Taylor expansion of the dispersion relation around $k_{0}(X)$ in the same manner as [20]. The precise meaning of all these quantities is discussed in detail in Section 2. The complex $X$-dependent coefficients $\omega_{0}(X)$ and $k_{0}(X)$ denote the usual local absolute frequency and wave number respectively, while $\omega_{k k}(X)$ is the second derivative of the linear dispersion relation with respect to the wave number $k$. The complex Landau "constant" $\gamma(X)$ is chosen so that nonlinearities are stabilizing everywhere (supercritical bifurcation), i.e., $\gamma_{i}(X) \equiv \operatorname{Im} \gamma(X)<0$ for all $X$. In the entire paper, the field $\psi(x, t)$ is assumed to be advected in the positive $x$-direction everywhere to mimic the dynamics of open flows. As demonstrated in Section 4.8, this assumption is equivalent to $k_{0, i}(X)<0$ for all $X$. Thus the increasing and decreasing $x$-directions will be referred to as "downstream" and "upstream" respectively.

Model (4) has been shown to successfully describe a large range of pattern formation phenomena $[26,15,32]$. Here it is used as an idealized representation of spatially developing flows, Similar conclusions are expected to hold for a general system governed by (1). The analytical results obtained in Sections 24 are further supported by numerical simulations of the CGL equation (4), 
as depicted in the accompanying figures. The remaining part of the paper (Section 5) is devoted to the complete higher-order asymptotic analyses of the various regions and layers appearing as ingredients in the spatial structure of global modes. The results are derived in the general context of system (1) by following a methodology analogous to that previously used by Pier and Huerre [33] and Bassom et al. [1]. This complete asymptotic analysis is rather technical and may be skipped in a first reading. The main conclusions are discussed in a final Section 6.

\section{Local instability properties}

Under the assumption that the governing equation only depends on space through the slow variable $X$, local characteristics of the medium are recovered by freezing $X$ in (1) and studying the corresponding strictly uniform medium. In the sequel, "local" always refers to properties of uniform systems obtained by extending the medium of a specific downstream station $X$ towards $x= \pm \infty$. At this local level of analysis, $X$ and $x$ are then considered to be independent: the fast component $x$ is involved in spatial differentiation whereas $X$ plays the part of an independent control parameter. The rigorous asymptotic analysis reestablishing the link between $x$ and $X$ via (3) in terms of WKBJ approximations [2] is postponed to Section 5.

In the present section the properties of infinite spatially uniform media governed by an equation of the form

$$
\frac{\partial \psi}{\partial t}=\mathcal{F}\left(\partial_{x}\right)[\psi]
$$

are reviewed. The results are applicable to any nonlinear operator $\mathcal{F}\left(\partial_{x}\right)=$ $\mathcal{F}\left(\partial_{x} ; X_{0}\right)$ derived from (1) for some fixed location $X=X_{0}$. Explicit forms are obtained for the uniform CGL equation

$$
i \frac{\partial \psi}{\partial t}=\left(\omega_{0}+\frac{1}{2} \omega_{k k} k_{0}^{2}\right) \psi+i \omega_{k k} k_{0} \frac{\partial \psi}{\partial x}-\frac{1}{2} \omega_{k k} \frac{\partial^{2} \psi}{\partial x^{2}}+\gamma|\psi|^{2} \psi
$$

The linear properties dictating the dynamics of small amplitude perturbations are routinely obtained. The main assumption used throughout the present study is that (5) admits a continuous family of nonlinear travelling waves. This is guaranteed as long as the nonlinearities are supercritically stabilizing, as demonstrated below.

Small amplitude perturbations are governed by the counterpart of (5) lin- 
earized around $\psi=0$,

$$
\frac{\partial \psi}{\partial t}=\mathcal{L}\left(\partial_{x}\right)[\psi]
$$

Any perturbation is a superposition of elementary waves $e^{i(k x-\omega t)}$ where the complex wave number $k$ and frequency $\omega$ satisfy the linear dispersion relation

$$
\omega=\Omega^{\ell}(k) \equiv i \mathcal{L}(i k) .
$$

For eq.(6), it takes the simple form

$$
\omega=\omega_{0}+\frac{1}{2} \omega_{k k}\left(k-k_{0}\right)^{2}
$$

where it is assumed that $\omega_{k k, i} \equiv \operatorname{Im} \omega_{k k}<0$ in order to enforce causality (see Section 2.3).

The dispersion relation (8) governs all linear properties of the system. Three situations are of particular interest: the temporal evolution problem, the impulse response, and the spatial response problem.

\subsection{Temporal evolution and nonlinear dispersion relation}

Consider the evolution of an initial spatially harmonic perturbation

$$
\psi(x, t=0)=A e^{i k x}+\text { c.c. }
$$

of real wave number $k$ and small amplitude $A \ll 1$. Its linear temporal growth rate is $\Omega_{i}^{\ell}(k) \equiv \operatorname{Im} \Omega^{\ell}(k)$. Two typical variations of $\Omega_{r}^{\ell}$ and $\Omega_{i}^{\ell}$ with $k$ are sketched by solid lines in Fig.1. When $\Omega_{i}^{\ell}(k)<0$, the wave exponentially decays in time. When $\Omega_{i}^{\ell}(k)>0$, the wave is temporally amplified and eventually governed by the full nonlinear equation (5). Assume that stabilizing nonlinearities lead to a fully nonlinear travelling wave of the form

$$
\psi(x, t)=\Psi(k x-\omega t ; k)
$$

where $\omega$ is a real frequency and the function $\Psi(\theta ; k)$ is $2 \pi$-periodic in $\theta$. This one-parameter family of nonlinear solutions parametrized by $k$ is characterized by the nonlinear dispersion relation

$$
\omega=\Omega^{n \ell}(k),
$$

represented by the dashed curves in Fig.1-a,c. The travelling waves (10) and dispersion relation (11) are the nonlinear counterparts of the linear normal modes $e^{i(k x-\omega t)}$ and dispersion relation (8). Since the medium is assumed to 

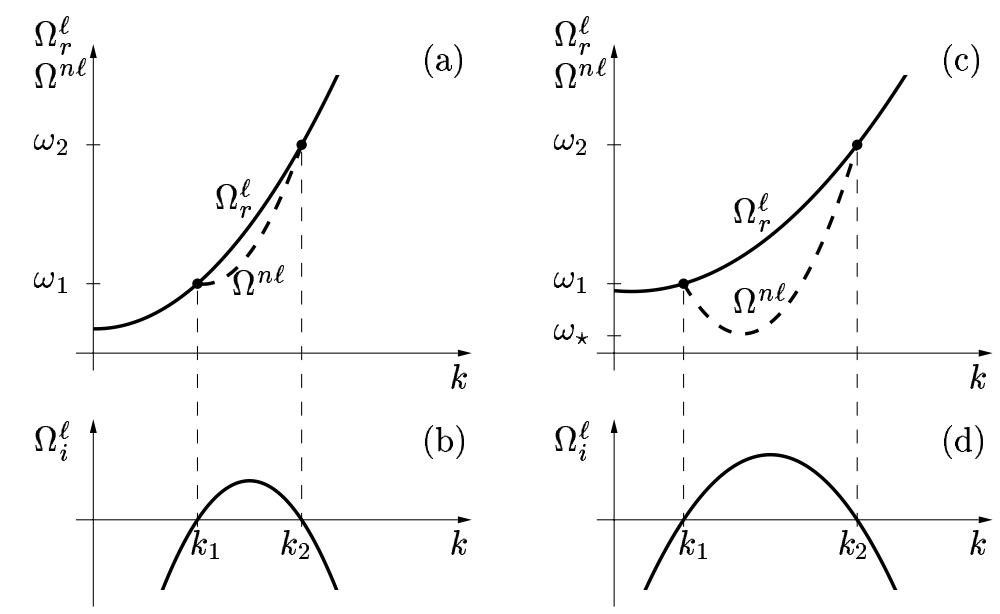

Fig. 1. Linear and nonlinear temporal branches of the CGL equation. (b,d) Temporal growth rate $\Omega_{i}^{\ell}(k)$ as a function of the real wave number. Unstable wave numbers lie in the range $k_{1}<k<k_{2}$. (a,c) The nonlinear temporal branch $\Omega^{n \ell}(k)$ (dashed curves) is defined in the unstable wave number range and is connected to the $\Omega_{r}^{\ell}(k)$ curve (solid lines) at the neutrally stable boundaries where $\omega_{1}=\Omega^{\ell}\left(k_{1}\right)=\Omega^{n \ell}\left(k_{1}\right)$ and $\omega_{2}=\Omega^{\ell}\left(k_{2}\right)=\Omega^{n \ell}\left(k_{2}\right)$. The nonlinear branch may be either monotonous (a) or exhibit an extremum (c).

be supercritical, nonlinear solutions $\Psi(\theta ; k)$ only exist in the unstable wave number range $k_{1}<k<k_{2}$ defined by $\Omega_{i}^{\ell}(k)>0$. Towards the boundaries of this range, the linear growth rate vanishes as well as the nonlinear saturation amplitude of $\Psi(\theta ; k)$. A weakly nonlinear calculation then shows that the higher harmonics of $\Psi(\theta)$ are slaved to the fundamental, and, in the neutrally stable limit, the nonlinear frequency equals the linear real frequency

$$
\Omega^{n \ell}(k)=\Omega^{\ell}(k) \quad \text { when } \quad \Omega_{i}^{\ell}(k)=0
$$

In Fig.1-a,c, the nonlinear temporal branches are seen to be connected to their linear counterparts at $\omega_{1}=\Omega^{n \ell}\left(k_{1}\right)=\Omega^{\ell}\left(k_{1}\right)$ and $\omega_{2}=\Omega^{n \ell}\left(k_{2}\right)=\Omega^{\ell}\left(k_{2}\right)$ respectively. In weakly unstable media, the unstable wave number range is small and in general the nonlinear frequency is a monotonous function of the wave number (Fig.1-a). Further above threshold, the unstable wave number range increases and the nonlinear temporal branch $\Omega^{n \ell}(k)$ may exhibit an extremum (Fig.1-c). As a result, one value of $\omega$ may be associated to two distinct wave numbers as further discussed in Section 2.5.

In general the functions $\Psi$ as well as $\Omega^{n \ell}$ cannot be calculated analytically, but they are easily obtained by performing a numerical simulation in a spatially periodic interval of wave length $2 \pi / k$. In the particular case of the CGL equation (6), nonlinear solutions are explicitly obtained as finite amplitude harmonic waves

$$
\psi(x, t)=R(k) \exp i\left[k x-\Omega^{n \ell}(k) t\right]
$$



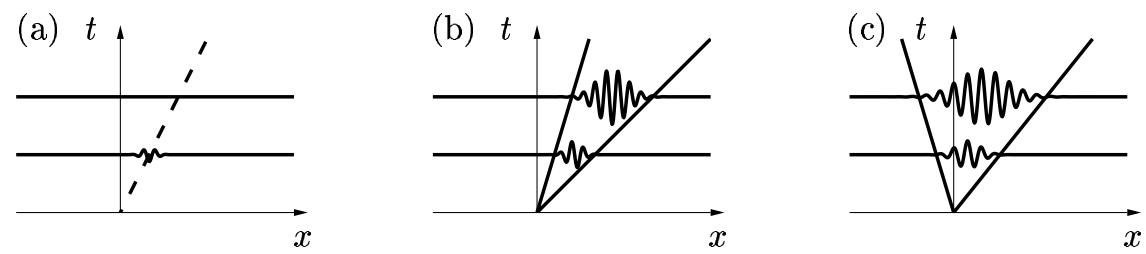

Fig. 2. Typical impulse response of (a) stable (b) convectively unstable (c) absolutely unstable medium.

with

$$
\Omega^{n \ell}(k)=\frac{\operatorname{Im}\left(\gamma^{\star} \Omega^{\ell}(k)\right)}{\operatorname{Im} \gamma^{\star}} \text { and } R^{2}(k)=\frac{\operatorname{Im}\left(\Omega^{\ell}(k)\right)}{\operatorname{Im} \gamma^{\star}},
$$

where the superscript $\star$ denotes the complex conjugate. Recall that the condition of stabilizing nonlinearities implies $\gamma_{i}<0$.

\subsection{Impulse response and absolute instability}

The impulse response as defined by many authors [4,3,20-22] is given by

$$
\frac{\partial \psi}{\partial t}=\mathcal{L}\left(\partial_{x}\right)[\psi]+\delta(x) \delta(t)
$$

where $\delta$ denotes the Dirac delta function. Different behaviors arise as illustrated in the $(x, t)$-plane in Fig.2.

The medium is said to be linearly stable (Fig.2-a) if the response $\psi(x, t)$ decays on all rays $x / t=\mathrm{C}^{\text {st }}$. This is fulfilled when

$$
\Omega_{i}^{\ell}(k)<0 \text { for all real } k
$$

In an unstable medium, at least one growing wavepacket develops from $(x, t)=$ $(0,0)$. If the growing wavepacket moves away from the source at $x=0$ and eventually leaves the medium unperturbed, the instability is said to be convective (Fig.2-b). If, by contrast, the instability grows in place and invades the system both upstream and downstream, the instability is said to be absolute (Fig.2-c). The convective or absolute nature of the instability depends on the absolute frequency $\omega_{0}$ associated with the absolute wave number $k_{0}$ observed along the ray $x=0$ defined as

$$
\omega_{0}=\Omega^{\ell}\left(k_{0}\right) \quad \text { where } \quad \frac{d \Omega^{\ell}}{d k}\left(k_{0}\right)=0
$$

The medium is absolutely unstable (AU) if $\omega_{0, i}>0$, convectively unstable $(\mathrm{CU})$ if $\omega_{0, i}<0$. The form (6) in which the CGL equation has been cast explicitly puts forward its dependence on $\omega_{0}$ and $k_{0}$. 


\subsection{Spatial response and causality}

Consider the response of the medium to a localized time harmonic forcing. The response to a forcing of real frequency $\omega_{f}$ and amplitude $A_{f}$, switched on at $t=0$, is governed by the signalling problem

$$
\frac{\partial \psi}{\partial t}=\mathcal{F}\left(\partial_{x}\right)[\psi]+\left[A_{f} \delta(x) H(t) e^{-i \omega_{f} t}+\text { c.c. }\right]
$$

with $H$ denoting the Heaviside unit step function.

For small amplitude forcing $\left(A_{f} \ll 1\right)$, the response in the neighborhood of the forcing location is governed by the linear spatial problem with $\mathcal{F}$ replaced by $\mathcal{L}$ in (15). Switching on the forcing at $t=0$ produces a transient wave packet together with the steady state response at the forcing frequency. Whenever the medium is stable or convectively unstable, transients decay or move away out of the system, and the long time response is established at the forcing frequency. When the medium is absolutely unstable, switch-on transients overwhelm the response at the forcing frequency and the signalling problem (15) is ill-posed [4,3]. Hence we only consider the spatial problem (15) for at most CU systems. The steady state response is made up of normal modes $e^{i\left(k x-\omega_{f} t\right)}$ satisfying $\omega_{f}=\Omega^{\ell}(k)$. This equation in general admits several solutions $k_{m}^{\ell}\left(\omega_{f}\right)$ indexed by $m$, the number of which very much depends on the particular form of $\Omega^{\ell}(k)$.

Causality requires that $\psi=0$ for all $t<0$. Using a residue calculation in the complex $\omega$-plane to solve $(15)$ with $\mathcal{F}$ replaced by $\mathcal{L}$, and assuming that temporal growth rates are bounded $\left(\max \left\{\Omega_{i}^{\ell}(k), k\right.\right.$ real $\}$ finite), it is readily shown $[4,3,20]$ that the spatial branches $k_{m}^{\ell}$ either pertain to the downstream $(x>0)$ or to the upstream $(x<0)$ response to forcing. The downstream (upstream) branches are denoted by $k_{m}^{\ell+}\left(k_{m}^{\ell-}\right)$. For a given real forcing frequency the distribution of the spatial branches $k_{m}^{\ell}(\omega)$ into + or - branches is derived, according to classical arguments [4,3], from an examination of the complete linear dispersion relation $\Omega^{\ell}(k)$ in the entire complex $k$-plane. In the sequel spatial branches are said to be causal + branches or causal - branches according to whether they prevail downstream $(x>0)$ or upstream $(x<0)$ of the forcing location. Hence causality always refers to the spatial response to a localized harmonic forcing.

For simplicity assume that $\Omega^{\ell}(k)$ exhibits a single second-order branch point $\omega_{0}$ with only two spatial branches $k^{\ell+}$ and $k^{\ell-}$, as in the case of the CGL dispersion relation (9) where

$$
k^{\ell \pm}(\omega)=k_{0} \pm \sqrt{2 \frac{\omega-\omega_{0}}{\omega_{k k}}} .
$$




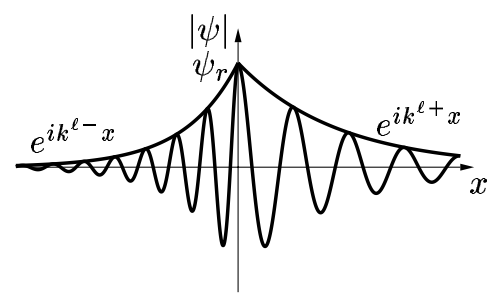

(a)

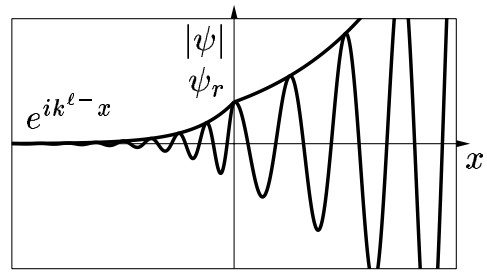

(b)
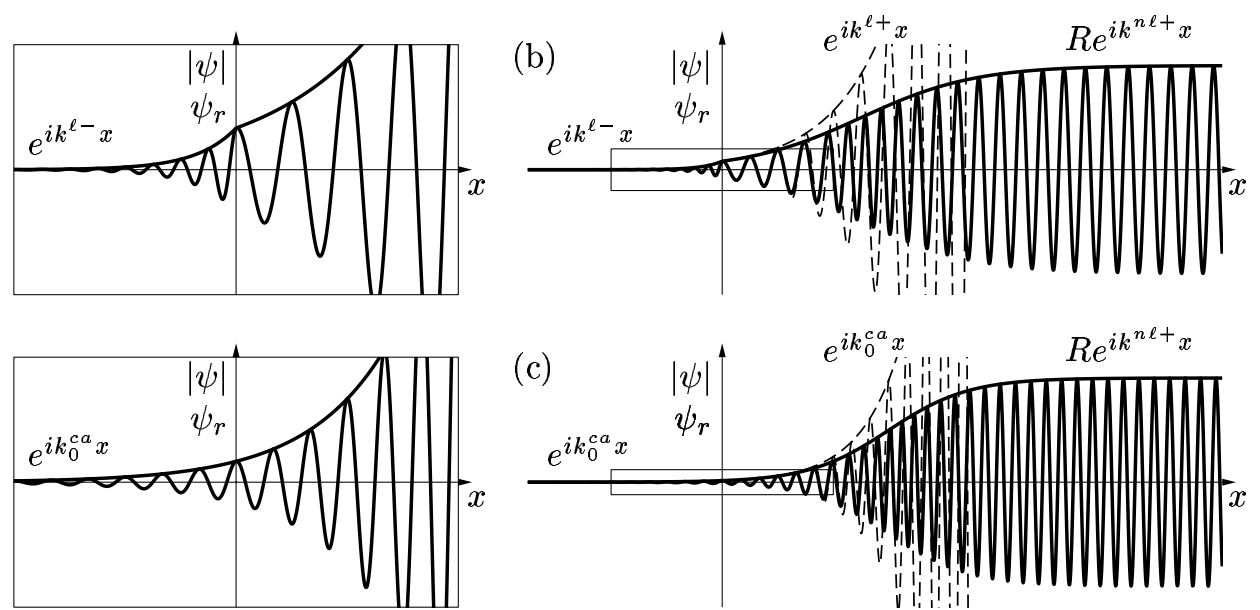

Fig. 3. Response to time-harmonic forcing applied at $x=0$. (a) Upstream and downstream decaying response in a stable or CU medium. (b) Linearly amplified downstream response and nonlinearly saturated solution in a CU medium. (c) Stationary front in a marginally absolutely unstable medium. Note that slope discontinuity at the forcing location has vanished. Dashed lines indicate exponentially growing branches in the linear approximation.

The spatial growth rate of the response depends on $k_{i}^{\ell} \equiv \operatorname{Im} k^{\ell}$. The downstream response decays for frequencies such that $k_{i}^{\ell+}(\omega)>0$; upstream decay occurs when $k_{i}^{\ell-}(\omega)<0$. This is always the case for stable media (Fig.3-a). Whenever a linear spatial $k^{\ell+}(\omega)$ branch is amplified, nonlinear terms have to be taken into account at some distance from the source, however small the forcing amplitude. When the response reaches finite amplitude, nonlinear saturation prevents further amplification and leads to a nonlinear travelling wave at the excitation frequency for some real wave number $k^{n \ell}$ (Fig.3-b). Since the nonlinear wave train is asymptotically reached far downstream of the source, it is denoted as $k^{n \ell+}(\omega)$. This nonlinear solution would also be obtained in a temporal evolution problem at the same wave number. Thus, the forcing frequency $\omega_{f}$ and the nonlinear response wave number $k^{n \ell+}$ again satisfy the nonlinear dispersion relation (11). Note that due to the choice of a basic advection towards $x=+\infty$, only the downstream response may be spatially amplified, and only the $k^{n \ell+}(\omega)$ branch is accessible via a forcing problem.

The precise discussion of the relationship between linear and nonlinear spatial branches is postponed to Section 2.5. 


\subsection{Stationary fronts as spatial response without forcing}

Many studies [16,38-41] have been devoted to the derivation of selection criteria for propagating fronts connecting an unstable $\psi=0$ state to a fully nonlinear saturated state in a uniform medium. In situations where the front velocity is linearly selected [16,39], the front moves towards its decaying edge in AU media, towards its finite amplitude edge in $\mathrm{CU}$ media. A stationary front is then precisely obtained when the medium is exactly at the CU/AU transition. The same stationary front solution may be recovered in the context of the spatial response to time-harmonic forcing as discussed below.

Consider the signalling problem (15) in a uniform medium

$$
\frac{\partial \psi}{\partial t}=\mathcal{F}\left(\partial_{x} ; X\right)[\psi]+\left[A_{f} \delta(x) H(t) e^{-i \omega_{f} t}+\text { c.c. }\right]
$$

where the frozen slow scale $X$ has been explicitly introduced as an external control parameter. Let us examine how the response to a localized forcing of frequency $\omega_{f}$ varies with the parameter $X$ which controls the instability properties of the medium. Assume that the medium is $\mathrm{CU}$ for $X<X^{c a}$ and marginally AU at $X=X^{c a}$, i.e., $\omega_{0, i}(X)<0$ for $X<X^{c a}$, and $\omega_{0}^{c a} \equiv \omega_{0}\left(X^{c a}\right)$ real. Let $k_{0}^{c a} \equiv k_{0}\left(X^{c a}\right)$ denote the complex absolute wave number at the $\mathrm{CU} / \mathrm{AU}$ transition. Since basic advection is assumed to be in the positive $x$-direction, $k_{0, i}^{c a}<0$, as shown in Section 4.8 .

Upon making use of the associated linear dispersion relation $\Omega^{\ell}$ and invoking continuity of the response at $x=0$, the exact long time linear response associated with the CGL equation (17) is obtained as

$$
\psi(x, t)=\frac{2 A_{f}}{\omega_{k k}(X)} \frac{\exp i\left[k^{\ell \pm}\left(\omega_{f}\right) x-\omega_{f} t\right]}{k^{\ell+}\left(X, \omega_{f}\right)-k^{\ell-}\left(X, \omega_{f}\right)}+\text { c.c. }
$$

The $e^{i k^{\ell+} x}$ and $e^{i k^{\ell-} x}$ branches naturally pertain to the regions $x>0$ and $x<0$ respectively. In order to obtain a normalized response such that $\max |\psi(0, t)|=$ $\alpha$, the forcing amplitude is adjusted to the level

$$
A_{f}\left(X, \omega_{f}\right)=\frac{\alpha}{4} \omega_{k k}(X)\left[k^{\ell+}\left(X, \omega_{f}\right)-k^{\ell-}\left(X, \omega_{f}\right)\right]
$$

If $\alpha \ll 1$, the linear response is guaranteed to remain valid in a neighborhood of $x=0$ even though the response may reach a finite amplitude further downstream.

If the medium is stable for large $X<0$, both upstream and downstream parts of the response decay, i.e., $k_{i}^{\ell+}\left(\omega_{f} ; X\right)>0$ and $k_{i}^{\ell-}\left(\omega_{f} ; X\right)<0$ for large $X<0$ (cf. Fig.3-a). As the control parameter $X$ and forcing frequency $\omega_{f}$ are varied continuously to approach the limit $X^{c a}, \omega_{0}^{c a}$, the downstream response $k^{\ell+}$ is 
eventually amplified, whereas the upstream branch $k^{\ell-}$ still decays (Fig.3b). Indeed, by definition of $\omega_{0}^{c a}$ [see also (16)] both spatial branches meet at $X=X^{c a}$ and $\omega_{f}=\omega_{0}^{c a}$, i.e.,

$$
k^{\ell+}\left(X^{c a}, \omega_{0}^{c a}\right)=k^{\ell-}\left(X^{c a}, \omega_{0}^{c a}\right)=k_{0}^{c a} .
$$

Since $k_{0, i}^{c a}<0$, it is therefore guaranteed that $k_{i}^{\ell+}\left(X, \omega_{f}\right)$ changes sign and becomes negative as $\left(X, \omega_{f}\right)$ approach $\left(X^{c a}, \omega_{0}^{c a}\right)$, while $k_{i}^{\ell-}\left(X, \omega_{f}\right)$ does not. In such a régime, the downstream growing response $\alpha \exp \left[i k^{\ell+}\left(X, \omega_{f}\right) x\right]$ reaches a finite amplitude at $x \sim(\ln \alpha) / k_{i}^{\ell+}>0$. At this station, the linearly growing wave $k^{\ell+}\left(X, \omega_{f}\right)$ is replaced by its nonlinear counterpart $k^{n \ell+}\left(X, \omega_{f}\right)$.

In the convectively unstable régime $\left(X<X^{c a}\right)$, both the spatial growth rate and wave number are discontinuous at $x=0$, i.e., $k^{\ell+} \neq k^{\ell-}$. The forcing location is then a singular point of the total response (Fig.3-a,b).

When $\left(X, \omega_{f}\right) \rightarrow\left(X^{c a}, \omega_{0}^{c a}\right)$, the medium approaches absolute instability and both branches $k^{\ell+}\left(X, \omega_{f}\right)$ and $k^{\ell-}\left(X, \omega_{f}\right)$ tend towards $k_{0}^{c a}$. Thus, in this process, the slope discontinuity in the response at $x=0$ smoothes out. Moreover, according to (19), the forcing amplitude $A_{f}(X)$ required to maintain the normalization condition $\max |\psi(0, t)|=\alpha$ vanishes. Thus in the marginally AU régime $X=X^{c a}$, a smooth stationary front of frequency $\omega_{f}=\omega_{0}^{c a}$ prevails without any forcing (Fig.3-c). This front directly connects the upstream linear $k^{\ell-}$ branch to the downstream nonlinear $k^{n \ell+}$ branch. As mentioned in Section 2.3, the + and - notations have causal meaning only in CU systems. The previous argument indicates that, in a marginally AU system, the two branches on both sides of a front are still determined by causal considerations through a continuation procedure from the CU side. From the above discussion, a stationary front in a spatially uniform system is obtained for zero-amplitude forcing whenever the medium becomes marginally $A U$ and the forcing frequency equals the corresponding real absolute frequency $\omega_{0}^{c a}$.

\subsection{Nature of nonlinear spatial branches: Cautionary remarks}

In Section 2.3 it has been demonstrated that, in the framework of a signalling problem, nonlinear wave trains are generated downstream of the forcing location in a CU system. Such a formulation naturally leads to a causal definition of spatial $k^{n \ell+}(\omega)$ branches. These fully nonlinear wave trains satisfy the nonlinear dispersion relation (11). Thus the nonlinear wave number $k^{n \ell}(\omega)$ may formally be obtained by solving (21) for a given frequency. In this subsection we discuss the precise relationship between linear and nonlinear spatial branches as illustrated in Fig.4, and introduce definitions of $k^{n \ell+}$ or $k^{n \ell-}$ branches which apply to all formal solutions of the nonlinear dispersion relation. In this process it will be seen that only the + superscript in CU systems may be assigned 

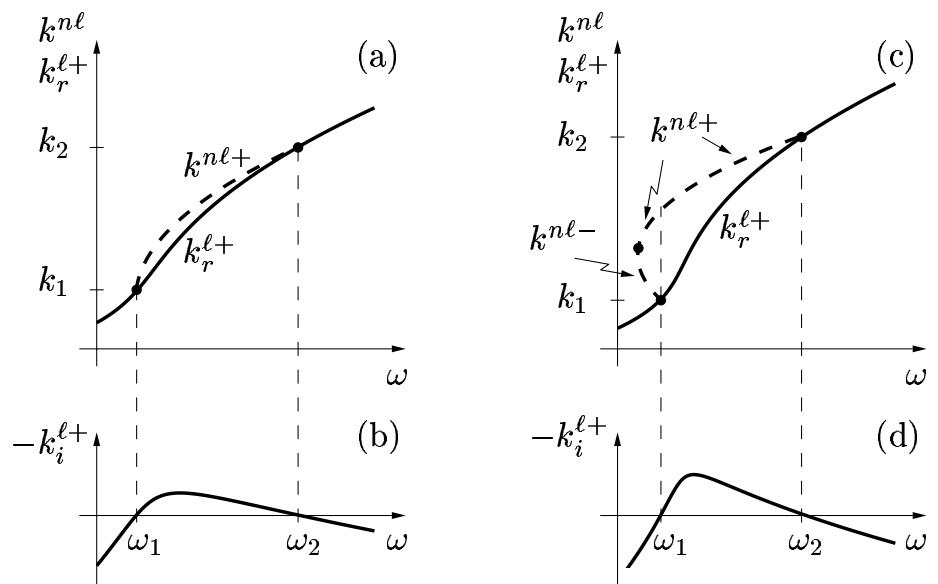

Fig. 4. Linear and nonlinear spatial branches of the CGL equation. (b,d) Spatial growth rate $-k_{i}^{\ell+}(\omega)$ of downstream spatial branch as a function of the real frequency. Unstable frequencies lie in the range $\omega_{1}<\omega<\omega_{2}$. The nonlinear spatial wave number $k^{n \ell}(\omega)$ may be either single-valued as in (a) or double-valued as in (c). (a) The unique nonlinear spatial branch $k^{n \ell+}(\omega)$ (dashed curve) is defined in the unstable frequency range and is connected to the $k_{r}^{\ell+}(\omega)$ curve (solid line) at the neutrally stable boundaries where $k_{1}=k^{\ell+}\left(\omega_{1}\right)=k^{n \ell+}\left(\omega_{1}\right)$ and $k_{2}=k^{\ell+}\left(\omega_{2}\right)=k^{n \ell+}\left(\omega_{2}\right)$. (c) Two nonlinear spatial branches $k^{n \ell+}(\omega)$ and $k^{n \ell-}(\omega)$ (dashed curve) coexist, which issue from the neutrally stable boundaries at $k_{1}$ and $k_{2}$ respectively and merge at a turning point (solid dot). Note that $k^{n \ell-}$ is now connected to $k^{\ell+}$ at $k_{1}$.

to nonlinear branches according to causality considerations.

Whereas the temporal problem yields the real nonlinear frequency as a function of real wave number as represented by the dashed lines in Fig.1, conversely the spatial problem yields the real nonlinear wave number as a function of real frequency. This inversion process is straightforward in situations where $\Omega^{n \ell}(k)$ is monotonous as in Fig.1-a. But it is more delicate in situations where $\Omega^{n \ell}(k)$ exhibits an extremum as in Fig.1-c.

In the first case (Fig.1-a), the behavior of the linear and nonlinear spatial branches is illustrated in Fig.4-a,b. The linear downstream response is specified by the spatial growth rate $-k_{i}^{\ell+}$ displayed in Fig.4-b. For forcing frequencies in the range $\omega_{1}<\omega<\omega_{2}$, the response is amplified and gives way to a nonlinear spatial solution of wave number $k^{n \ell+}$ as indicated by the dashed line in Fig.4a. Note that, as in the temporal case (Fig.1-a), the nonlinear branch $k^{n \ell+}$ is connected to its linear counterpart $k^{\ell+}$ at the neutrally stable frequencies $\omega_{1}$ and $\omega_{2}$.

In the second case (Fig.1-c), the behavior of the linear and nonlinear spatial branches is as illustrated in Fig.4-c,d. The presence of an extremum in $\Omega^{n \ell}(k)$ (dashed line in Fig.1-c) is responsible for the double-valuedness of $k^{n \ell}(\omega)$ (dashed line in Fig.4-c). In the linearly unstable range $\omega_{1}<\omega<\omega_{2}$ 
there is still a single nonlinear wave number $k^{n \ell+}(\omega)$ which governs the far downstream nonlinear response to forcing, and it is legitimately assigned the superscript + with a causal meaning. As in the first case, it is connected to a linear $k^{\ell+}$ branch at the neutral frequency $\omega_{2}$. Note however that for $\omega<\omega_{1}$, two nonlinear $k^{n \ell}$ branches coexist. Following the definition adopted by the front community [41] let us introduce the "nonlinear group velocity" $d \Omega^{n \ell}(k) / d k$. The $+(-)$ superscript is then given to the spatial $k^{n \ell}$ branch with positive (negative) nonlinear group velocity. With this convention, the + superscript is assigned to the extension of the $k^{n \ell+}$ branch down to the turning point (solid dot in Fig.4-c), whereas the - superscript is assigned to the remaining part of the $k^{n \ell}$ curve. It is now this $k^{n \ell-}$ curve that is connected to the linear $k^{\ell+}$ branch at the neutral frequency $\omega_{1}$. It is essential to note that the nonlinear branches $k^{n \ell+}$ and $k^{n \ell-}$ may not be accessible via forcing at frequencies $\omega<\omega_{1}$. They are therefore not necessarily causal and the + and - superscripts are purely chosen for notational convenience in this frequency range.

For infinitesimally small forcing level, the wave number observed far downstream as the forcing frequency $\omega$ is varied follows the decaying complex $k^{\ell+}(\omega)$ for $\omega<\omega_{1}$ or $\omega>\omega_{2}$, and the real $k^{n \ell+}(\omega)$ in the range $\omega_{1}<\omega<\omega_{2}$. Hence the transition between a linear and a nonlinear wave train is continuous at $\omega_{2}$ where $k^{n \ell+}$ and $k^{\ell+}$ merge, but discontinuous at $\omega_{1}$ with a consequent jump in wave number.

This spatial analysis only holds for CU media. In AU media, switch-on transients spread and grow in situ. Long time evolution is thus completely contaminated by transients and no steady state response at the forcing frequency may be identified. However solving the linear and nonlinear dispersion relations (8) and (11) for a given frequency still yields $k^{\ell}(\omega)$ and $k^{n \ell}(\omega)$ solutions. These linear and nonlinear branches then loose their causal meaning. But the corresponding nonlinear wave trains may still be generated from a temporal problem and the superscripts + and - may be assigned to the nonlinear branches $k^{n \ell}(\omega)$ via the sign of $d \Omega^{n \ell} / d k$.

\section{Spatial variations of local instability properties}

The previous results derived for spatially uniform media also yield the local linear and nonlinear instability characteristics of weakly nonuniform media, provided that the control parameter $X$ now be interpreted as the slow streamwise coordinate. The respective dispersion relations at each station $X$ read

$$
\begin{aligned}
& \omega=\Omega^{\ell}(k, X), \quad \omega \text { and } k \text { complex, } \\
& \omega=\Omega^{n \ell}(k, X), \quad \omega \text { and } k \text { real. }
\end{aligned}
$$


The local linear dispersion relation pertains to any complex wave number whereas the local nonlinear dispersion relation is defined only for real wave numbers associated with a positive temporal growth rate $\Omega_{i}^{\ell}(k, X)>0$. In strictly uniform media, linear normal modes are sought in the form $\psi=$ $A \exp i\left[k^{\ell}(\omega) x-\omega t\right]+$ c.c. In weakly nonuniform media, such modes are replaced by

$$
\psi=A(X) \exp \left(\frac{i}{\epsilon} \int^{X} k^{\ell}(u, \omega) d u-i \omega t\right)+\text { c.c. }
$$

As demonstrated in the classical WKBJ procedure outlined in Section 5, the local linear wave number $k^{\ell}(X, \omega)$ necessarily satisfies the local linear dispersion relation (20). In strictly uniform media, nonlinear travelling waves are sought in the form $\Psi\left[k^{n \ell}(\omega) x-\omega t\right]$. In weakly nonuniform media, such travelling waves are replaced by slowly modulated wave packets of the form

$$
\psi \sim \Psi\left(\frac{1}{\epsilon} \int^{X} k^{n \ell}(u, \omega) d u-\omega t+\Theta(X) ; k^{n \ell}(X, \omega), X\right)
$$

where the local nonlinear wave number $k^{n \ell}(X, \omega)$ satisfies the nonlinear local dispersion relation (21). The slowly varying functions $A(X)$ and $\Theta(X)$ appearing in (22) and (23) respectively are obtained in the complete asymptotic analysis (Section 5).

The objective of this section is then to study the changing topology of linear complex $k^{\ell}$ and nonlinear real $k^{n \ell}$ spatial branches as the global real frequency is varied.

\subsection{Instability domains}

Let us first introduce the regions of local convective or absolute instability in physical $X$-space and determine the domain of existence of nonlinear wave trains in $(X, k)$-space. The local absolute frequency $\omega_{0}(X)$ and wave number $k_{0}(X)$ are derived from the local linear dispersion relation (20) as in (14). The local convective or absolute nature of the medium is determined by the sign of $\omega_{0, i}(X)$. In a typical situation of interest, absolute instability occurs in a central finite domain. Consider $\omega_{0}(X)$ to be of the form sketched in the complex $\omega$-plane in Fig. 5 -a: $\omega_{0, i}(X)$ is an increasing-decreasing function of $X$ with a single maximum $\omega_{0, i}^{\max }$ reached at $X=X^{\max }$.

Whenever $\omega_{0, i}^{\max }>0$, there exists a finite $A U$ domain, $X^{c a}<X<X^{a c}$, defined as the region where $\omega_{0, i}(X)>0$. Its boundaries $X^{c a}$ and $X^{a c}$ are the stations where the local absolute frequency is real, $\omega_{0}^{c a} \equiv \omega_{0}\left(X^{c a}\right)$ and $\omega_{0}^{a c} \equiv \omega_{0}\left(X^{a c}\right)$ 

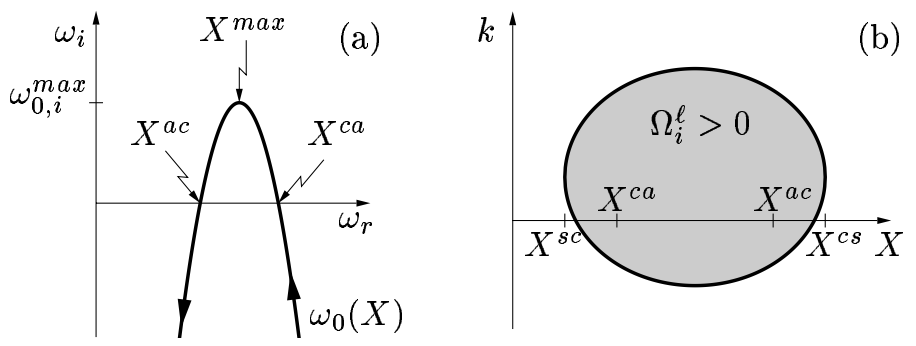

Fig. 5. (a) Locus of the local absolute frequency $\omega_{0}(X)$ in the complex frequency plane for $-\infty<X<+\infty$. The AU interval $X^{c a}<X<X^{a c}$ is associated with $\omega_{0, i}(X)>0$. (b) Nonlinear balloon in the $(X, k)$-plane defined by $\Omega_{i}^{\ell}(k, X)>0$ and bounded by the curve of neutral stability $\Omega_{i}^{\ell}(k, X)=0$. In the $X$-direction the balloon spans the domain of local linear instability $X^{s c}<X<X^{c s}$ and extends beyond the AU interval.

respectively.

The domain of local instability, characterized by unstable real wave numbers, $\Omega_{i}^{\ell}(k, X)>0$, defines the nonlinear balloon in the $(X, k)$-plane (Fig.5-b). In the $X$-direction the balloon extends beyond the AU domain to the stations of linear neutral stability, denoted $X^{s c}$ and $X^{c s}$. At each unstable location, the nonlinear balloon extends in the $k$-direction over the local unstable wave number range. Note that, due to causality, large wave numbers are always temporally decaying; thus the nonlinear balloon is necessarily bounded in the $k$-direction.

A typical system therefore displays the following structure: a central AU domain $X^{c a}<X<X^{a c}$ of finite extent, surrounded by two CU regions $X^{s c}<X<X^{c a}$ and $X^{a c}<X<X^{c s}$, which in turn are embedded in two semiinfinite stable regions extending to $X= \pm \infty$. As mentioned in Section 4.1, one may relax the stability requirement at $X= \pm \infty$ and the medium may remain $\mathrm{CU}$ to $X= \pm \infty$.

\subsection{Linear spatial branches}

The loci of linear spatial branches $k^{\ell}(X, \omega)$ as functions of $X$ is now qualitatively discussed in the complex $k$-plane for different values of the complex frequency $\omega$. Such an analysis will illustrate the relationship between the behavior of linear spatial branches and the local CU/AU properties of the medium. Linear spatial branches $k^{\ell}(X, \omega)$ are obtained by solving the local linear dispersion relation (20) for a given frequency $\omega$. In the case of the CGL equation (4) they read

$$
k^{\ell \pm}(X, \omega)=k_{0}(X) \pm \sqrt{2 \frac{\omega-\omega_{0}(X)}{\omega_{k k}(X)}}
$$


In Section 2.3 the choice of the + and - branches was shown to be dictated by causality for real frequencies in stable or CU media. Thus the $k^{\ell+}$ and $k^{\ell-}$ branches are unambiguously defined in the distinct regions $X<X^{c a}$ and $X>X^{a c}$ for real frequencies. The fate of spatial branches in the AU range $X^{c a}<X<X^{a c}$ is now examined for different frequencies in the complex $\omega$-plane as illustrated in Fig.6.

As a result of causality [4,3], the spatial branches $k^{\ell \pm}(X, \omega)$ do not cross the $k_{r}$-axis in the complex $k$-plane for large enough $\omega_{i}>0$. For such frequencies far above the absolute frequency curve $\left\{\omega_{0}(X)\right\}$ (cf. Fig.6-a), the $k^{\ell+}\left(k^{\ell-}\right)$ branch is globally defined as the one located in the upper (lower) half $k$-plane for all real $X$. The $k^{\ell \pm}(X, \omega)$ branches continuously deform as $\omega$ is varied. They may cross the $k_{r}$-axis for finite values of $X$ (Fig.6-b,c,d), but remain in the same half $k$-plane for $X \rightarrow \pm \infty$. As $\omega$ is kept above the curve $\left\{\omega_{0}(X)\right\}$ (denoted by $\omega \succ\left\{\omega_{0}(X)\right\}$ ), no branch switching may occur as readily seen by inspection of eq.(24). This property yields definitions of the $k^{\ell \pm}$ branches that remain uniformly valid in $X$, for all complex frequencies $\omega \succ\left\{\omega_{0}(X)\right\}$, even though the medium may be locally AU.

When $\omega \in\left\{\omega_{0}(X)\right\}$, say $\omega=\omega_{0}\left(X_{0}\right)$, the two $k^{\ell \pm}$ branches pinch at $k_{0}\left(X_{0}\right)$ for $X=X_{0}$ (Fig.6-e illustrates the case of particular interest where $X_{0}=X^{c a}$ ). For frequencies $\omega \prec\left\{\omega_{0}(X)\right\}$ below the absolute frequency curve (Fig.6-f), the continuous $k^{\ell}$ curves connect the upper and lower half $k$-planes as $X$ is varied from $-\infty$ to $+\infty$. Global $k^{\ell \pm}$ branches can no longer be defined, but the + and - superscripts may still be assigned according to causality in the distinct $X<X^{c a}$ and $X>X^{a c}$ regions represented by thick lines in Fig.6. For $\omega \prec\left\{\omega_{0}(X)\right\}$ (Fig.6-f), the $k^{\ell-}$ branch for $X<X^{c a}$ is connected to the $k^{\ell+}$ branch in the region $X>X^{a c}$, across the AU domain (part of the curve being represented by a thin line).

These considerations apply in particular to real $\omega$. For frequencies outside the $\omega_{0}^{c a}-\omega_{0}^{a c}$ interval, i.e., $\omega \succ\left\{\omega_{0}(X)\right\}$, the $k^{\ell+}$ and $k^{\ell-}$ branches are globally defined, although causality considerations do not apply in the central AU domain. For frequencies in the $\omega_{0}^{c a}-\omega_{0}^{a c}$ interval, i.e., $\omega \prec\left\{\omega_{0}(X)\right\}$, the $k^{\ell+}$ and $k^{\ell-}$ branches turn one into the other across the AU domain.

\subsection{Nonlinear spatial branches}

The goal of this section is to describe synthetically the qualitative properties of the nonlinear spatial branches $k^{n \ell}(X, \omega)$ as functions of $X$ for different values of the real frequency $\omega$. Finite amplitude waves are governed by the nonlinear dispersion relation (21). Figure 7-a,b illustrates its properties by projecting the surface defined as $\omega=\Omega^{n \ell}(k, X)$ in the $(X, k, \omega)$-space onto the $(X, k)$ and 

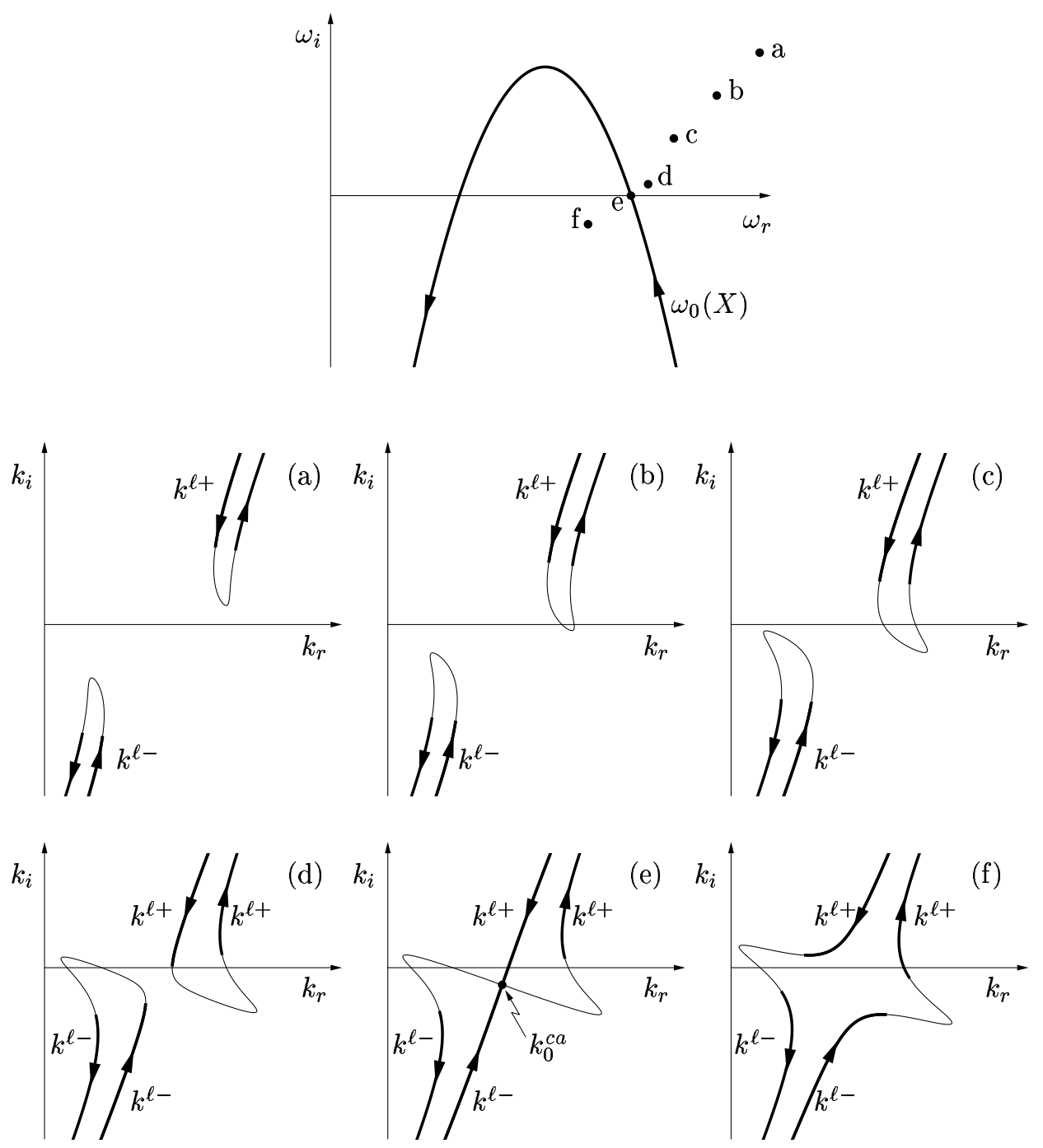

Fig. 6. (a-f) Loci of linear spatial branches $k^{\ell}(X, \omega)$ of CGL equation in the complex $k$-plane for frequencies $\omega$ indicated in the complex frequency plane on top sketch. Arrows on the curves indicate direction of increasing $X$. Thick lines pertain to the stable or CU regions $X<X^{c a}$ and $X>X^{a c}$, thin lines to the central AU region $X^{c a}<X<X^{a c}$. (a-d) For frequencies $\omega \succ\left\{\omega_{0}(X)\right\}$, the spatial branches $k^{\ell \pm}(X)$ remain distinct and are located in the same half $k$-plane for $X \rightarrow \pm \infty$, but they may cross the $k_{r}$-axis for finite values of $X$. As the frequency approaches the $\omega_{0}(X)$ curve, the spatial branches move closer to each other. (e) For a frequency located on the $\omega_{0}(X)$ curve, here $\omega=\omega_{0}\left(X^{c a}\right)$, pinching occurs at the corresponding absolute wave number, here at $k=k_{0}^{c a}$, when $X=X^{c a}$. (f) When $\omega \prec\left\{\omega_{0}(X)\right\}$, the continuous curves connect the upper and lower half $k$-planes as $X \rightarrow \pm \infty$. Definition as $k^{\ell \pm}$ branches still holds in the distinct CU domains $X<X^{c a}$ and $X>X^{a c}$, but not in the central AU region.

$(X, \omega)$ planes respectively. Nonlinear spatial branches $k^{n \ell}(X, \omega)$ for a given frequency $\omega$ are obtained as the intersections of this surface with planes of constant frequency. 

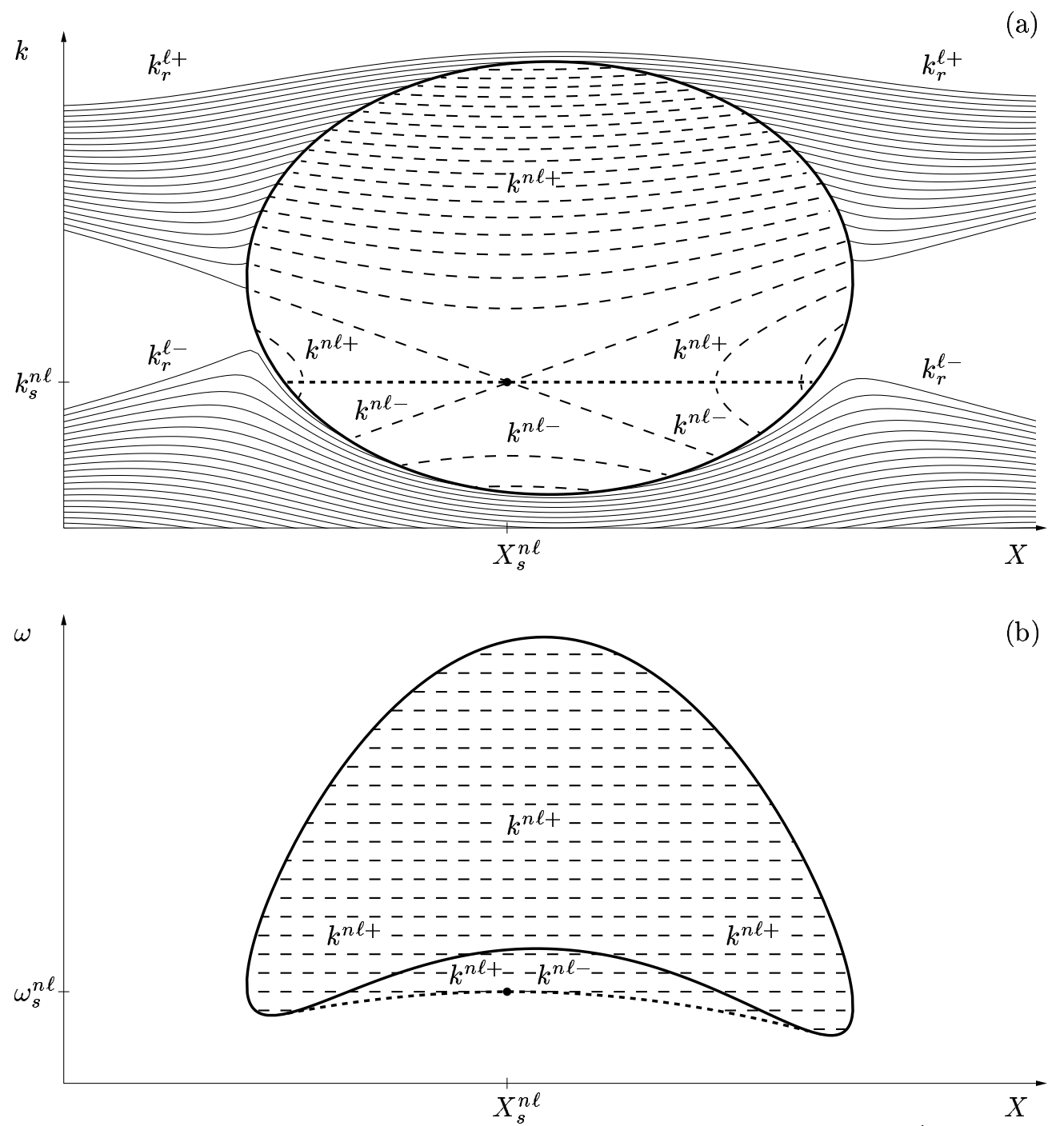

Fig. 7. Projections of the nonlinear dispersion relation surface $\omega=\Omega^{n \ell}(k, X)$ on the $(X, k)$ - and $(X, \omega)$-planes. Nonlinear spatial branches $k^{n \ell+}(X, \omega)$ and $k^{n \ell-}(X, \omega)$ represented by constant frequency level curves (dashed lines) live within the neutral stability boundary (thick solid line). Similarly marked curves in (a) and (b) map one into the other. (a) Nonlinear spatial branches display a saddle structure and, by convention, $k^{n \ell+}$ and $k^{n \ell-}$ refer to the upper and lower regions on either side of the steepest descent curve (thick dotted line) emerging from the saddle point $X_{s}^{n \ell}, k_{s}^{n \ell}$ (solid dot). The real parts $k_{r}^{\ell \pm}$ of the linear spatial branches (thin solid lines) are also shown outside the nonlinear balloon for the same frequencies as the nonlinear branches. Note their continuous connection at the neutral stability boundary. (b) In the $(X, \omega)$-plane, the $k^{n \ell+}$ and $k^{n \ell-}$ branches give rise to two overlapping sheets joining along a fold curve (thick dotted line). Within the neutral stability boundary (solid curve), only the $k^{n \ell+}$ sheet exists. Between the fold line and the neutral curve, both $k^{n \ell+}$ and $k^{n \ell-}$ sheets coexist. The saddle point located at $X_{s}^{n \ell}$ corresponds to the frequency $\omega_{s}^{n \ell}$. 
Nonlinear wave trains only exist inside the nonlinear balloon of the $(X, k)$ plane, defined by $\Omega_{i}^{\ell}(k, X)>0$ (see Fig.5-b). Its neutrally stable boundary characterized by $\Omega_{i}^{\ell}(k, X)=0$ is represented by the thick closed curve in Fig.7-a. Since the nonlinear dispersion relation $\omega=\Omega^{n \ell}(k, X)$ is always a single-valued function of $k$, the mapping in the $(X, k)$-plane (Fig.7-a) is oneto-one and all the nonlinear solutions are contained inside the neutral stability boundary.

By contrast, $k^{n \ell}(X, \omega)$ is not ensured to be a single-valued function of $\omega$, the projection of the same surface in the $(X, \omega)$-plane (Fig.7-b) may therefore display an overlap region represented by two sheets that are connected at a fold, as indicated by the thick dotted line. In this view, the region inside the neutral curve (thick line) corresponds to a unique wave number $k^{n \ell+}(X, \omega)$ of the nonlinear dispersion relation, whereas the domain between the neutral curve and the fold corresponds to two distinct sheets associated with two wave numbers $k^{n \ell \pm}$. The image of the fold line in the $(X, k)$-plane is also represented by a thick dotted line in Fig.7-a.

The nonlinear spatial branches $k^{n \ell}(X, \omega)$ of real frequency $\omega$ are obtained as the level contours $\Omega^{n \ell}(k, X)=\mathrm{C}^{\text {st }}$ indicated by thin dashed lines in both figures. In Fig.7-b they are just straight lines. Depending on the frequency, these straight lines may intersect either only the single-valued domain, or both domains. The double-valued nature of the nonlinear spatial branches merely reflects features already encountered in the uniform case in Section 2.5 (Fig.4-c). We have deliberately chosen a configuration displaying this doublevaluedness, which is made manifest here by the saddle point structure in the contour levels $\Omega^{n \ell}(k, X)=\mathrm{C}^{\text {st }}$ of Fig.7-a. Following the definition adopted in Section 2.5, the nonlinear spatial branches are labelled $k^{n \ell+}$ and $k^{n \ell-}$ according to the sign of the "nonlinear group velocity" $\partial \Omega^{n \ell} / \partial k$. Accordingly, the $k^{n \ell+}$ and $k^{n \ell-}$ domains of Fig.7-a precisely correspond to the two sheets of Fig.7-b connected via the thick dashed fold line.

In order to emphasize the relationship between linear and nonlinear branches, the linear spatial branches $k^{\ell \pm}(X, \omega)$ have also been displayed by thin solid lines in Fig.7-a through their real part $k_{r}^{\ell \pm}(X, \omega)$ at the same frequencies. Note that linear branches continuously connect to nonlinear branches at the neutral stability boundary. This property is not surprising: At the neutral stability boundary in the $(X, k)$-plane, the linear branch $k^{\ell}(X, \omega)$ is purely real and equals its nonlinear counterpart $k^{n \ell}(X, \omega)$ so that

$$
k^{n \ell}(X, \omega)=k_{r}^{\ell}(X, \omega) \quad \text { and } \quad k_{i}^{\ell}(X, \omega)=0
$$

Equivalently,

$$
\Omega^{n \ell}(k, X)=\Omega_{r}^{\ell}(k, X) \text { and } \Omega_{i}^{\ell}(k, X)=0 .
$$


As discussed in Section 3.2 (Fig.6), the linear spatial branches are globally defined as $k^{\ell+}$ or $k^{\ell-}$ for $\omega \succ\left\{\omega_{0}(X)\right\}$. While lowering the frequency, starting from large positive values, the $k^{\ell+}$ and/or $k^{\ell-}$ branch may cross the $k_{r}$-axis in the complex $k$-plane (Fig.6-b,c,d). As this linear wave number branch moves into the opposite half $k$-plane, it becomes spatially amplified and gives birth to a nonlinear branch. This corresponds in the $(X, k)$-plane to the emergence of a $k^{n \ell}$ branch connected at the boundary of the nonlinear balloon to a $k_{r}^{\ell}$-branch (Fig.7-a).

By further lowering the frequency, switching between the nonlinear branches may take place, as implied by the saddle structure of the dashed curves in Fig.7-a. When the frequency decreases, pinching between $k^{n \ell-}$ and $k^{n \ell+}$ will take place for $\omega=\omega_{s}^{n \ell}$ at the saddle point $\left(X_{s}^{n \ell}, k_{s}^{n \ell}\right)$ of the nonlinear dispersion relation $(21)$ in the $(X, k)$-plane. Below this saddle-point frequency, the nonlinear spatial branches move into the left- and right-hand sectors bounded by the saddle-point asymptotes (Fig.7-a). For a given frequency in this range, nonlinear spatial branches are indeed generated at the boundary of the nonlinear balloon but they fail to exist in the heart of the nonlinear region surrounding the saddle-point. This behavior is provoked by the merging of $k^{n \ell+}$ and $k^{n \ell-}$ on the fold line (thick dotted line of Fig.7-a) and their subsequent disappearance. The implications of such a nonlinear saddle point structure on global mode selection is discussed in Section 4.6.

\section{Globally synchronized structures}

Having investigated local instability properties of the spatially developing medium, we now turn to the study of globally synchronized solutions of system (1) and associated bifurcations. Such global modes are defined as stationary time-periodic solutions satisfying

$$
\psi\left(x, t+2 \pi / \omega_{g}\right)=\psi(x, t)
$$

where $\omega_{g}$ is the global real frequency. Frequency selection criteria for selfsustained global oscillations are derived below and their spatial structure is obtained. The properties of the medium which dictate the selected global mode type are identified and the ensuing bifurcations are analyzed as global control parameters are varied.

\subsection{Boundary conditions}

To completely determine the global mode problem, proper boundary conditions in connection with eq.(1) have to be specified. Global modes are defined 
as intrinsic oscillations which are due to the dynamics of the central region and not to perturbations invading the system from $X= \pm \infty$. Consequently the boundary conditions must be causal: The solution close to the boundaries is necessarily dictated by the intrinsic oscillations occurring in the central region. Thus, far downstream near $X=+\infty$ the solution is necessarily made up of a + branch caused by the dynamics governing the central region upstream of it. Similarly the solution necessarily involves a - branch towards $X=-\infty$. Such boundary conditions at $X= \pm \infty$ will be referred to as causal.

When the medium is assumed to be stable in the far downstream and upstream regions, causal boundary conditions are equivalent with decaying ones. Indeed, in the stable regions no nonlinear solutions exist. Causality then requires a linear $k^{\ell+}\left(k^{\ell-}\right)$ branch towards $X=+\infty(-\infty)$. Due to stability, $k_{i}^{\ell+}>0$ and $k_{i}^{\ell-}<0$, thus the solution necessarily decays towards $X= \pm \infty$.

However the medium may remain $\mathrm{CU}$ up to $X= \pm \infty$. In such instances, decaying boundary conditions are not necessarily fulfilled. However causality still holds: if the solution remains fully nonlinear down to $X=+\infty$, it is there necessarily made up of the $k^{n \ell+}$ branch only. Thus proper boundary conditions for the global mode problem do not necessarily require exponential decay: they should however satisfy causality.

Since a global mode is a solution over the entire $X$-axis, it necessarily connects a - branch at $X=-\infty$ to a + branch at $X=+\infty$. This crossover from - to + branches may only be achieved for certain frequencies. The search for global modes is thus a nonlinear eigenvalue problem for the global frequency $\omega_{g}$. The manner in which this crossover takes place in the central region gives rise to different types of global modes as described below.

\subsection{Steep global modes}

According to [34], the spatial structure of steep global modes is characterized by the presence of a sharp front at the upstream boundary $X^{c a}$ of the $\mathrm{AU}$ region, as sketched in Fig.8-a.

This front at the location of marginal absolute instability $\left[\omega_{0, i}\left(X^{c a}\right)=0\right]$ is precisely of the type obtained in Section 2.4: It oscillates at the real absolute frequency $\omega_{g}=\omega_{0}^{c a} \equiv \omega_{0}\left(X^{c a}\right)$ and allows a crossover between the upstream $k^{\ell-}$ and the downstream $k^{n \ell+}$ branches. The exponential decay of the upstream tail of the front is determined by the imaginary part of the corresponding absolute wave number $k_{0}^{c a} \equiv k_{0}\left(X^{c a}\right)$. The upstream $k^{\ell-}\left(X, \omega_{g}\right)$ branch extending towards $X=-\infty$ is precisely generated at the pinch point $X=X^{c a}$ in the complex $k$-plane (Fig.8-b). The downstream nonlinear wake of the front is made up of the $k^{n \ell+}\left(X, \omega_{g}\right)$ branch. As depicted in the $(X, k)$-plane 

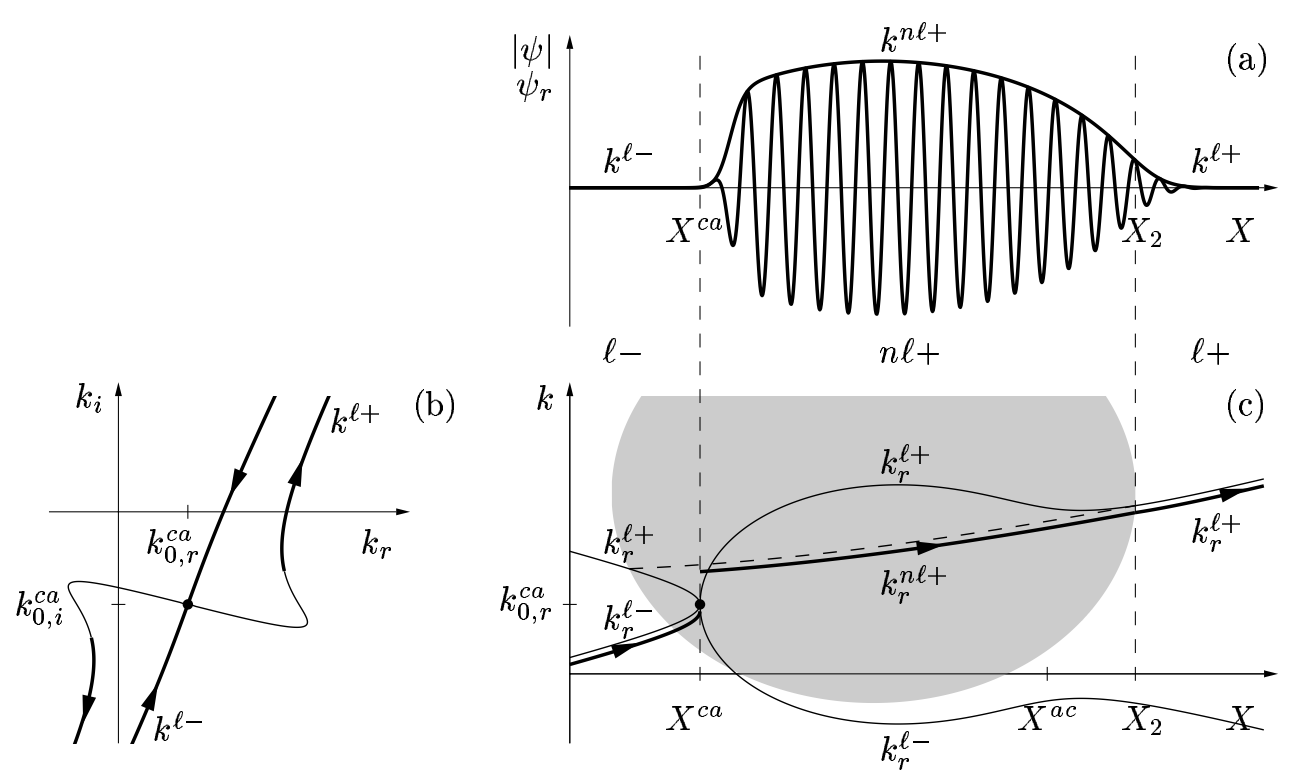

Fig. 8. Structure of steep global mode in CGL equation. (a) Envelope $|\psi|$ and real part $\psi_{r}$ as functions of downstream distance $X$. The sharp front located at the upstream boundary $X^{c a}$ of $\mathrm{AU}$ region initiates the fully nonlinear development extending down to the neutral station $X_{2}$. (b) Linear spatial branches $k^{\ell \pm}(X)$ of steep global frequency $\omega_{0}^{c a}$ in the complex $k$-plane. Pinching occurs for the absolute wave number $k_{0}^{c a}$ at $X=X^{c a}$. Thick lines pertain to the stable or CU regions $X<X^{c a}$ and $X>X^{a c}$, thin lines to the central AU region $X^{c a}<X<X^{a c}$. (c) Corresponding linear $k_{r}^{\ell \pm}$ (solid) and nonlinear $k^{n \ell+}$ (dashed) spatial branches in the $(X, k)$-plane. Local wave number of steep global mode in (a) follows path indicated by thick line. Three domains are identified: the $k^{\ell-}$ branch prevails in the upstream linear $\ell-$ region $X<X^{c a}$; the front at $X^{c a}$ is associated with a jump in wave number and in its wake the $k^{n \ell+}$ branch develops in the fully nonlinear $n \ell+$ domain $X^{c a}<X<X_{2}$ extending towards the boundary of the nonlinear balloon (shaded); the $k^{\ell+}$ branch continuously takes over in the linear $\ell+$ region downstream of the neutral station $X_{2}$.

of Fig.8-c, nonlinear travelling waves prevail in the region $X^{c a}<X<X_{2}$ extending down to the location $X_{2}$ where the $k^{n \ell+}$ branch meets the boundary of the nonlinear balloon in the $(X, k)$-plane. At the neutrally stable station $X_{2}$ the amplitude of the nonlinear travelling wave vanishes and the linear branch $k^{\ell+}\left(X, \omega_{g}\right)$ continuously takes over in the downstream linear region $X>X_{2}$.

The following frequency selection criterion then holds: the steep global frequency $\omega_{g}$ is given by the real absolute frequency $\omega_{0}^{c a}$ prevailing at the front location $X^{c a}$ separating the $\mathrm{CU}$ and AU regions. In other words

$$
\omega_{g}=\omega_{0}\left(X^{c a}\right) \quad \text { and } \quad \omega_{0, i}\left(X^{c a}\right)=0
$$

The front at $X^{c a}$ effectively acts as a wave maker for the entire flow. It may be interpreted as a local oscillator inducing the linear upstream - branch and the nonlinear downstream + branch. 
It should be noted that the steep global mode criterion (25) is also fulfilled by the absolute frequency $\omega_{0}^{a c}$ prevailing at the downstream boundary $X^{a c}$ of the $\mathrm{AU}$ region. Thus, whenever $\omega_{0, i}^{\max }>0$, two steep global modes exist: one of frequency $\omega_{0}^{c a}$ with a front at the upstream boundary $X^{c a}$ of the AU region and one of frequency $\omega_{0}^{a c}$ with a front at its downstream boundary $X^{a c}$ (see Fig.5a). When $\omega_{0, i}^{\max }<0$, no AU region is present and no steep global mode exists. Thus $\omega_{0, i}^{\max }$ constitutes the global control parameter governing the existence of steep global modes. When $\omega_{0, i}^{\max }$ is varied, transition to steep global modes occurs via a saddle-node bifurcation at $\omega_{0, i}^{\max }=0$ as demonstrated below.

The spatial structure of a solution with a front at $X^{c a}$ has been detailed in the previous subsection. The structure of a solution with a front at $X^{a c}$ is similar. Indeed, both linear $k^{\ell \pm}\left(X, \omega_{0}^{a c}\right)$ branches equal $k_{0}^{a c} \equiv k_{0}\left(X^{a c}\right)$ at $X=X^{a c}$. Due to the assumption $k_{0, i}<0$, the $k^{\ell-}$ branch is again exponentially damped while the $k^{\ell+}$ branch is amplified. As a result, nonlinear travelling waves $k^{n \ell+}$ are only present downstream of the front $\left(X>X^{a c}\right)$ and linear damped waves $k^{\ell-}$ upstream of the front $\left(X<X^{a c}\right)$. Thus the nonlinear part of such a global mode extends in the downstream $\mathrm{CU}$ region, whereas the central $\mathrm{AU}$ domain is covered by an exponentially decaying upstream tail.

Let us now show that an upstream front is a stable configuration whereas a downstream front is unstable. Consider a small displacement of the upstream front from its equilibrium location $X^{c a}$ towards $X>X^{c a}$. The front now experiences a slightly AU medium, hence, according to Dee and Langer [16], the nonlinear part grows and the front propagates towards its decaying edge, i.e., upstream. When this front is displaced towards $X<X^{c a}$, it penetrates into a CU region and is thus pushed downstream. In any case the front is seen to return to its equilibrium position $X^{c a}$.

On the contrary, a downstream front displaced from its equilibrium position $X^{a c}$ towards the AU region $X<X^{a c}$ continues to propagate upstream and completely invades the AU domain. When the front is displaced towards the $\mathrm{CU}$ region $X>X^{a c}$, it is swept away downstream towards $X=+\infty$. A downstream front is therefore unstable.

Thus when $\omega_{0, i}^{\max }>0$, a pair of steep global modes exists: the mode with a front at the upstream (resp. downstream) boundary of the AU region is stable (resp. unstable). In the limit $\omega_{0, i}^{\text {max }} \downarrow 0$, the AU domain shrinks and the front locations move in closer to each other, $X^{c a} \uparrow X^{\max }$ and $X^{a c} \downarrow X^{\text {max }}$. When $\omega_{0, i}^{\max }=0$ both front frequencies $\omega_{0}^{c a}$ and $\omega_{0}^{a c}$ equal $\omega_{0}\left(X^{\max }\right)$, and both steep global modes coincide. When $\omega_{0, i}^{\max }<0$, the domain is nowhere AU and no steep global mode exists. 
This behavior is typical of a saddle node bifurcation: while decreasing the bifurcation parameter $\omega_{0, i}^{\max }$, a stable and an unstable solution meet and disappear at the critical value $\omega_{0, i}^{\max }=0$.

Note that in general the steep global mode remains fully nonlinear for all $\omega_{0, i}^{\max }>0$. Indeed, for $0<\omega_{0, i}^{\max } \ll 1$ the extent of the convectively unstable domain remains $\mathcal{O}(1)$ (in terms of $X$ ) and so does the nonlinear region where the global mode lives.

\subsection{Linear global modes}

The linear global instability of the unperturbed $\psi=0$ state has been studied by Chomaz et al. [7] and Le Dizès et al. [25]. The instability properties were derived from an analytic continuation of the local absolute frequency $\omega_{0}(X)$ in the complex $X$-plane, as summarized below.

Linear global modes are assumed to be of the form $\psi(x, t)=\phi(X) e^{-i \omega_{g} t}$ of complex global frequency $\omega_{g}$. The spatial function $\phi$ is defined over the complex $X$-plane and the local complex wave number satisfies the linear dispersion relation (20) with complex $X$. A linear global mode is entirely made up of linear spatial branches $k^{\ell \pm}$. Due to causal boundary conditions, the $k^{\ell-}$ branch prevailing near $X=-\infty$ must necessarily connect to the $k^{\ell+}$ branch near $X=+\infty$. This can be achieved at a saddle point $X_{s}^{\ell}$ of the absolute frequency in the complex $X$-plane ${ }^{1}$. The frequency of the linear global mode is then equal to the saddle point frequency $\omega_{s}^{\ell}$, defined by

$$
\omega_{s}^{\ell}=\omega_{0}\left(X_{s}^{\ell}\right) \quad \text { where } \quad \frac{d \omega_{0}}{d X}\left(X_{s}^{\ell}\right)=0
$$

or equivalently by

$$
\omega_{s}^{\ell}=\Omega^{\ell}\left(k_{s}^{\ell}, X_{s}^{\ell}\right) \quad \text { where } \quad \frac{\partial \Omega^{\ell}}{\partial k}\left(k_{s}^{\ell}, X_{s}^{\ell}\right)=\frac{\partial \Omega^{\ell}}{\partial X}\left(k_{s}^{\ell}, X_{s}^{\ell}\right)=0
$$

Linear instability is determined by the sign of $\omega_{s, i}^{\ell} \equiv \operatorname{Im} \omega_{s}^{\ell}$; when $\omega_{s, i}^{\ell}<0$ (resp. $\omega_{s, i}^{\ell}>0$ ) the state $\psi=0$ is linearly stable (resp. unstable).

\footnotetext{
${ }^{1}$ Only linear global modes with a double turning point are considered here. The reader is referred to Le Dizès et al. [25] for a detailed analysis of linear global modes with two simple turning points.
} 


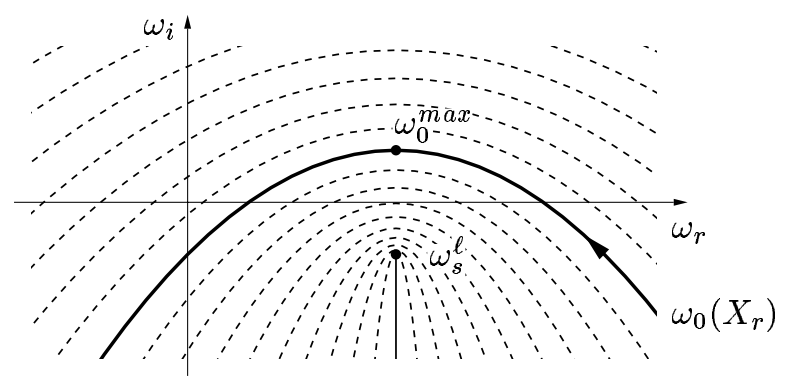

Fig. 9. Curves $\omega_{0}\left(X_{r}+i X_{i}\right)$ in the complex frequency plane for different values of $X_{i}$ when $X_{r}$ varies along the entire real axis. Bold curve pertains to $X_{i}=0$. The linear saddle point frequency $\omega_{s}^{\ell}$ is obtained at a cusp of this set of curves and is seen to lie below $\omega_{0}^{\max }$, towards the center of curvature of the $\omega_{0}\left(X_{r}\right)$ curve.

\subsection{Hysteresis}

The existence of nonlinear steep global modes is determined by the sign of $\omega_{0, i}^{\max }$, while the linear global instability of the unperturbed state $\psi=0$ is dictated by the sign of $\omega_{s, i}^{\ell}$. It is now shown qualitatively that always $\omega_{0, i}^{\max } \geq \omega_{s, i}^{\ell}$, and in general $\omega_{0, i}^{\max }>\omega_{s, i}^{\ell}$. Thus steep global modes may exist in globally linearly stable media (situations where $\omega_{s, i}^{\ell}<0<\omega_{0, i}^{\max }$ ), and the saddle node bifurcation implies hysteretic behavior as $\omega_{0, i}^{\max }$ is varied.

The relative position of $\omega_{0, i}^{\max }$ and $\omega_{s, i}^{\ell}$ is most conveniently illustrated in the complex frequency plane as sketched in Fig.9. The solid curve represents $\omega_{0}(X)$ for real $X$. The AU region corresponds to the $X$-interval over which $\omega_{0}(X)$ lies in the $\omega_{i}>0$ half-plane. The maximum $\omega_{0, i}^{\max }$ is reached at $X=X^{\max }$. Thus steep global modes exist whenever the curve $\omega_{0}(X)$ crosses the real $\omega$-axis. The complex frequency $\omega_{s}^{\ell}$ is obtained at a saddle point of the analytically continued function $\omega_{0}(X)$. For most situations of physical interest, $\omega_{0, i}(X)$ is an increasing-decreasing function on the real $X$-axis, while $\omega_{0, r}(X)$ is simply a smooth function. The dashed curves of Fig.9 represent the loci of $\omega_{0}\left(X_{r}+i X_{i}\right)$ for different values of $X_{i}$ when $X_{r}$ varies along the entire real axis. The saddle point frequency $\omega_{s}^{\ell}$ is obtained at a cusp of this set of curves and lies below the $\omega_{0}\left(X_{r}\right)$ curve, towards its center of curvature. Thus it follows clearly that $\omega_{0, i}^{\max }>\omega_{s, i}^{\ell}$ and one recovers the well known result that global linear instability requires an $\mathrm{AU}$ region of finite extent $[7,25]$.

In the neighborhood of $\omega_{0}^{\max }$, the absolute frequency $\omega_{0}(X)$ may be approximated by the Taylor expansion

$$
\omega_{0}(X) \sim \omega_{0}^{\max }+\omega_{0 X}\left(X-X^{\max }\right)+\frac{1}{2} \omega_{0 X X}\left(X-X^{\max }\right)^{2}
$$

with $\omega_{0 X, i}=0$ and $\omega_{0 X X, i}<0$. The linear saddle point, solution of $(26)$, is 


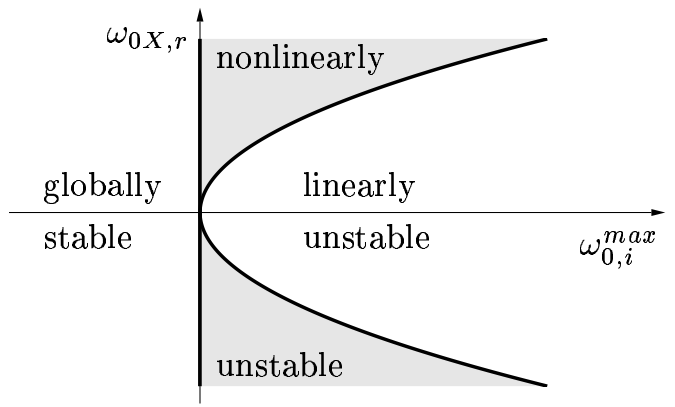

Fig. 10. Global linear and nonlinear stability in the $\left(\omega_{0, i}^{\max }, \omega_{0 X, r}\right)$-plane. Global stability is governed by the sign of $\omega_{0, i}^{\max }$. When $\omega_{0, i}^{\max }>0$ the medium is globally nonlinearly unstable although linearly stable in the gray region.

then explicitly given by

$$
X_{s}^{\ell}=X^{\max }-\frac{\omega_{0 X, r}}{\omega_{0 X X}} \quad \text { and } \quad \omega_{s}^{\ell}=\omega_{0}^{\max }-\frac{1}{2} \frac{\left(\omega_{0 X, r}\right)^{2}}{\omega_{0 X X}}
$$

thus

$$
\operatorname{Im} \omega_{s}^{\ell}=\omega_{0, i}^{\max }+\frac{1}{2} \frac{\left(\omega_{0 X, r}\right)^{2}}{\left|\omega_{0 X X}\right|^{2}} \omega_{0 X X, i}
$$

Only if $d \omega_{0, r}\left(X^{\max }\right) / d X=0$, does the saddle point $X_{s}^{\ell}$ coincide with $X^{\max }$ on the real $X$-axis and, under such a condition, $\omega_{s}^{\ell}=\omega_{0}\left(X^{\max }\right)$. The difference between $\omega_{0, i}^{\max }$ and $\omega_{s, i}^{\ell}$ is seen to depend on the magnitude of $\omega_{0 X, r} \equiv$ $d \omega_{0, r}\left(X^{\max }\right) / d X$ and to scale as $\left(\omega_{0 X, r}\right)^{2}$. The influence of the two parameters $\omega_{0, i}^{\max }$ and $\omega_{0 X, r}$ on global linear and nonlinear instability is illustrated in Fig.10.

The following scenario holds as the global control parameter $\omega_{0, i}^{\max }$ is varied at a fixed $\omega_{0 X, r}$ setting. When $\omega_{0, i}^{\max }<0$ (left-hand half-plane of Fig.10), the unperturbed state is stable, no nonlinear global mode exists. When $\omega_{0, i}^{\max }>0$ while $\omega_{s, i}^{\ell}<0$ (shaded region of Fig.10), a pair of steep global modes exist, one of which is stable, the other unstable. However the unperturbed state is still linearly globally stable ${ }^{2}$, but nonlinearly unstable. Only for $\omega_{0, i}^{\max }$ large enough such that $\omega_{s, i}^{\ell}>0$ (clear region inside parabola of Fig.10), does the basic state become linearly unstable. Whereas the global saddle node bifurcation is controlled by $\omega_{0, i}^{\max }$, the extent of the hysteresis range in $\omega_{0, i}^{\max }$ is governed by a second control parameter, namely $\omega_{0 X, r}$.

\footnotetext{
${ }^{2}$ The unperturbed state is however locally unstable.
} 

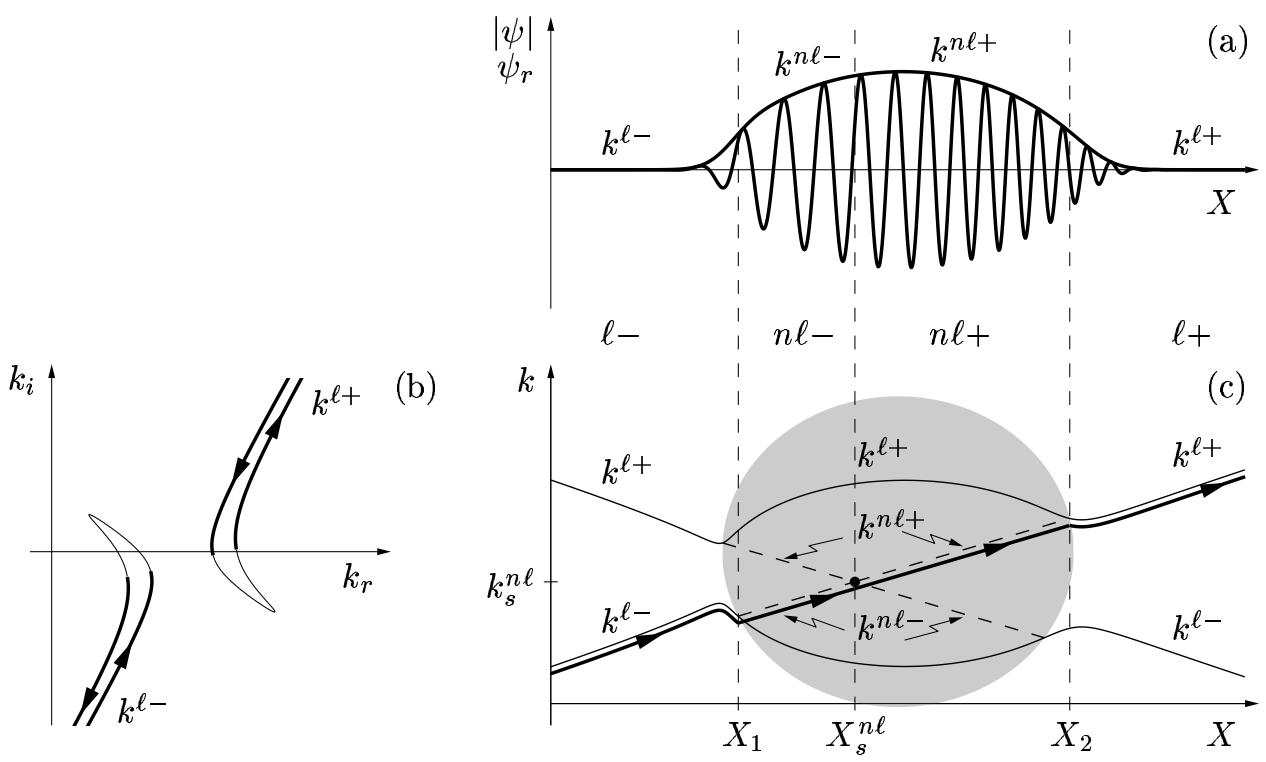

Fig. 11. Structure of soft global mode in CGL equation. (a) Overall smoothly varying envelope $|\psi|$ and real part $\psi_{r}$ as functions of downstream distance $X$. (b) Linear spatial branches $k^{\ell \pm}(X)$ of nonlinear saddle point frequency $\omega_{s}^{n \ell}$ in the complex $k$-plane. Both linear branches cross the $k_{r}$-axis and give birth to their nonlinear counterparts at the respective neutral stations. Thick lines pertain to the stable or $\mathrm{CU}$ regions $X<X^{c a}$ and $X>X^{a c}$, thin lines to the central AU region $X^{c a}<X<X^{a c}$. (c) Corresponding linear $k_{r}^{\ell \pm}$ (solid) and nonlinear $k^{n \ell \pm}$ (dashed) spatial branches in the $(X, k)$-plane. Pinching of nonlinear branches occurs for $k_{s}^{n \ell}$ at $X=X_{s}^{n \ell}$ in the core of the nonlinear region. Local wave number of soft global mode in (a) follows path indicated by thick line. Four domains are identified: the central nonlinear $n \ell-$ and $n \ell+$ regions prevail upstream and downstream of $X_{s}^{n \ell}$ where the nonlinear spatial branches $k^{n \ell-}$ and $k^{n \ell+}$ meet; at the upstream $X_{1}$ and downstream $X_{2}$ boundaries of the nonlinear balloon (shaded) the nonlinear spatial branches are continuously connected to their respective linear counterparts $k^{\ell \pm}$ prevailing in the semi-infinte linear regions $\ell-$ and $\ell+$ respectively.

\subsection{Soft global modes}

The existence of soft global modes has been analyzed in an earlier study [33]. Their structure is now briefly summarized, as well as their relationship to steep global modes.

An example is illustrated in Fig.11-a. In contrast to steep modes, no front is present and soft modes display an overall smoothly varying envelope and wave number. The connection between the upstream - branch and the downstream + branch occurs here in the core of the nonlinear region, at a saddle point of the nonlinear dispersion relation (21).

As already discussed in Section 3.3, the nonlinear branches $k^{n \ell \pm}(X, \omega)$, formally defined as the level contours $\Omega^{n \ell}(k, X)=\mathrm{C}^{\text {st }}$, may display a saddle 
point point $\left(X_{s}^{n \ell}, k_{s}^{n \ell}\right)$ in the $(X, k)$-plane (solid dot in Fig.7-a). More precisely, pinching of the nonlinear branches, defined by the condition

$$
k^{n \ell+}\left(X_{s}^{n \ell}, \omega_{s}^{n \ell}\right)=k^{n \ell-}\left(X_{s}^{n \ell}, \omega_{s}^{n \ell}\right)=k_{s}^{n \ell},
$$

then occurs at the real station $X_{s}^{n \ell}$ for the real saddle point frequency $\omega_{s}^{n \ell}$ of

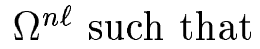

$$
\omega_{s}^{n \ell}=\Omega^{n \ell}\left(k_{s}^{n \ell}, X_{s}^{n \ell}\right) \quad \text { and } \quad \frac{\partial \Omega^{n \ell}}{\partial k}\left(k_{s}^{n \ell}, X_{s}^{n \ell}\right)=\frac{\partial \Omega^{n \ell}}{\partial X}\left(k_{s}^{n \ell}, X_{s}^{n \ell}\right)=0,
$$

with the saddle condition

$$
\left(\frac{\partial^{2} \Omega^{n \ell}}{\partial k \partial X}\left(k_{s}^{n \ell}, X_{s}^{n \ell}\right)\right)^{2}-\frac{\partial^{2} \Omega^{n \ell}}{\partial k^{2}}\left(k_{s}^{n \ell}, X_{s}^{n \ell}\right) \frac{\partial^{2} \Omega^{n \ell}}{\partial X^{2}}\left(k_{s}^{n \ell}, X_{s}^{n \ell}\right)>0 .
$$

Note the formal analogy of this nonlinear saddle point criterion (30) with its linear counterpart (27) which involves in general complex values of $k_{s}^{\ell}$ and $X_{s}^{\ell}$. The upstream $k^{n \ell-}\left(X, \omega_{s}^{n \ell}\right)$ branch and downstream $k^{n \ell+}\left(X, \omega_{s}^{n \ell}\right)$ branch are precisely initiated at the nonlinear saddle point $X_{s}^{n \ell}$, as depicted in Fig.11-c. These nonlinear travelling waves prevail in the range $X_{1}<X<X_{2}$, where $X_{1}$ and $X_{2}$ denote neutral stability stations of frequency $\omega_{s}^{n \ell}$, at the boundary of the nonlinear balloon in the $(X, k)$-plane. At $X_{1}$ and $X_{2}$, the amplitudes of the nonlinear travelling waves $k^{n \ell-}$ and $k^{n \ell+}$ respectively vanish and give way to their linear counterparts $k^{\ell-}$ and $k^{\ell+}$ respectively.

For the CGL equation (4) with $\omega_{0}(X)$ of the form (28), all other coefficients being kept uniform in $X$, the nonlinear saddle point location is explicitly obtained as

$$
\begin{gathered}
X_{s}^{n \ell}=X^{m a x}+\frac{\gamma_{i} \omega_{0 X, r}}{\operatorname{Im}\left(\gamma^{\star} \omega_{0 X X}\right)}, \\
k_{s}^{n \ell}=k_{0, r}+\frac{\operatorname{Re} \gamma^{\star} \omega_{k k}}{\operatorname{Im} \gamma^{\star} \omega_{k k}} k_{0, i} .
\end{gathered}
$$

and the soft global mode frequency reads

$$
\omega_{s}^{n \ell}=\omega_{0, r}^{\max }-\frac{\gamma_{r}}{\gamma_{i}} \omega_{0, i}^{\max }+\frac{\gamma_{i}\left(\omega_{0 X, r}\right)^{2}}{2 \operatorname{Im}\left(\gamma^{\star} \omega_{0 X X}\right)}+\frac{\left|\gamma^{\star} \omega_{k k}\right|^{2}}{2 \gamma_{i} \operatorname{Im}\left(\gamma^{\star} \omega_{k k}\right)} k_{0, i}^{2} .
$$

Condition (31) requires that $\left(\operatorname{Im} \gamma^{\star} \omega_{k k}\right)\left(\operatorname{Im} \gamma^{\star} \omega_{0 X X}\right)<0$.

It was shown in [33] that in situations where

$$
\operatorname{Im}\left(\gamma^{\star} \omega_{k k}\right)>0
$$

the nonlinear branches $k^{n \ell \pm}$ exist in the neighborhood of $X_{s}^{n \ell}$ for frequencies such that $\omega>\omega_{s}^{n \ell}$. When $\omega \downarrow \omega_{s}^{n \ell}$, the branches pinch at $k_{s}^{n \ell}$ for $X=X_{s}^{n \ell}$; and 
for $\omega<\omega_{s}^{n \ell}$, they fail to be defined around $X_{s}^{n \ell}$. In the following it is always assumed that the possible frequencies for the nonlinear spatial branches lie in the range $\omega>\omega_{s}^{n \ell}$. The opposite situation is exactly analogous and applies when $\operatorname{Im}\left(\gamma^{\star} \omega_{k k}\right)<0$. From (31) and (35), the following inequality is then derived

$$
\operatorname{Im}\left(\gamma^{\star} \omega_{0 X X}\right)<0
$$

\subsection{Transition between soft and steep global modes}

The selection mechanisms governing steep and soft global modes are now compared.

The steep criterion (25) only involves the boundary of the AU domain on the physical $X$-axis, regardless of the characteristics of the regions $X \neq X^{c a}$. Nevertheless a steep global mode only exists if the nonlinear $k^{n \ell+}\left(X, \omega_{0}^{c a}\right)$ branch can be followed from $X^{c a}$ down to the boundary of the nonlinear balloon and if it can there be continuously connected to the linear branch $k^{\ell+}\left(X, \omega_{0}^{c a}\right)$. In contrast, the soft criterion (30), involves a saddle point of the nonlinear dispersion relation. Again, a soft global mode only exists if the nonlinear spatial branches issuing from this nonlinear pinch point may effectively be continued via corresponding linear $k^{\ell \pm}$ branches in the respective downstream and upstream linear domains.

The selection of either steep or soft global modes depends not only on the local criteria $(25,30)$ but also on the necessity to enforce the boundary conditions. The key argument in the following derivation is based on a careful monitoring of the linear wave number branches in the complex $k$-plane and of their nonlinear counterparts in the $(X, k)$-plane as the global frequency is varied. The connection between linear and nonlinear spatial branches is shown to crucially depend on the relative magnitude of the characteristic frequencies $\omega_{s}^{n \ell}$ and $\omega_{0}^{c a}$. In all instances, only one global mode, steep or soft, is capable of continuously converting $k^{\ell-}$ at $X=-\infty$ to $k^{\ell+}$ at $X=+\infty$, as $X$ is varied. The two main scenarii of interest are illustrated in Fig.12 and 13 respectively, as discussed below.

Since nonlinear global modes necessarily involve real frequencies, let us focus on frequencies on the real $\omega$-axis. As shown in Section 3.2, for large positive or negative $\omega$ the linear spatial branches do not cross the $k_{r}$-axis in the complex $k$-plane. Since nonlinear spatial branches are assumed to exist for $\omega>\omega_{s}^{n \ell}$, we start with large positive values of the frequency.

As $\omega$ is decreased, the $k^{\ell \pm}(X, \omega)$ branches move in closer to each other and one or both cross the $k_{r}$-axis for finite values of $X$. A change in sign of $k_{i}^{\ell}$ sig- 
Fig. 12. (facing page) Evolution of CGL spatial branches with decreasing real frequency in a situation where the soft global mode is selected. The left sequence illustrates the evolution of the real part of linear branches $k_{r}^{\ell \pm}(X, \omega)$ (solid lines) and of nonlinear branches $k^{n \ell \pm}(X, \omega)$ (dashed lines) in the $(X, k)$-plane. Connecting points between linear and nonlinear branches are indicated with tick marks. In the right sequence, corresponding complex linear $k^{\ell \pm}(X, \omega)$ branches are sketched in the complex $\left(k_{r}, k_{i}\right)$-plane; superimposed on the same graphs are the curves $\left(k^{n \ell \pm}, R^{2}\right)$ of the nonlinear spatial branches. (a,b) For frequencies $\omega>\omega_{s}^{n \ell}$ linear $k^{\ell \pm}$ spatial branches successively cross the $k_{r}$-axis in the complex $k$-plane and give birth to the nonlinear $k^{n \ell \pm}$ branches between the corresponding neutral stations. (c) For the nonlinear saddle point frequency $\omega_{s}^{n \ell}$, nonlinear spatial branches pinch at $k_{s}^{n \ell}$ when $X=X_{s}^{n \ell}$. This pinch point joining $k^{n \ell-}$ and $k^{n \ell+}$ branches gives rise to a soft global mode connecting the $k^{\ell-}$ branch near $X=-\infty$ to the $k^{\ell+}$ branch prevailing near $X=+\infty$. (d,e) For frequencies $\omega<\omega_{s}^{n \ell}$, the nonlinear spatial branches fail to exist in the neighborhood of $X_{s}^{n \ell}$, but linear $k^{\ell \pm}$ branches are still defined. (e) When $\omega=\omega_{0}^{c a}$, the linear branches in turn pinch at $k=k_{0}^{c a}$ at the upstream boundary $X^{c a}$ of $\mathrm{AU}$ region. However, this pinch point is not associated with a steep global mode since the $k^{n \ell+}$ branch prevailing for $X>X^{c a}$ is not connected to the $k^{\ell+}$ branch extending to $X=+\infty$.

nifies that the corresponding branch is spatially growing: downstream spatial growth for $k_{i}^{\ell+}<0$ and upstream spatial growth for $k_{i}^{\ell-}>0$. As already mentioned (Section 2.3) a spatially growing branch $k^{\ell \pm}$ gives way to its nonlinear counterpart $k^{n \ell \pm}$ as a neutrally stable station is crossed in the $(X, k)$-plane.

Nonlinear $k^{n \ell}$ branches are by construction always real-valued. However, to illustrate their relationship with the linear $k^{\ell}$ branches they are also represented in the complex $k$-planes of Fig.12 and 13. For clarity their missing imaginary 

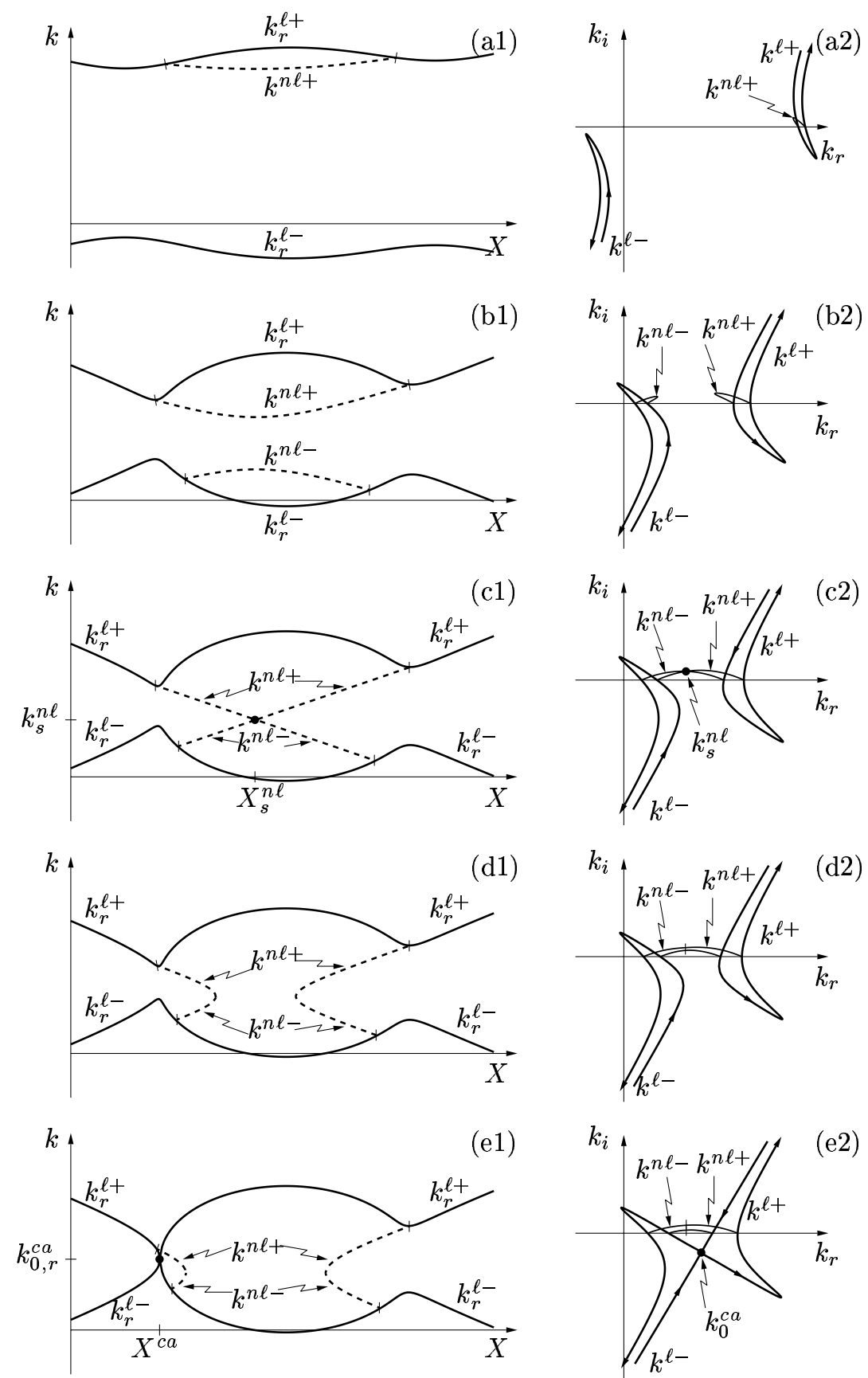

part is replaced by the square of the amplitude of the corresponding nonlinear solution. This avoids the collapse of the $k^{n \ell}$ branches onto the $k_{r}$-axis, and brings to the fore the continuity between linear and nonlinear branches at the neutral stations where $k_{i}^{\ell}$ as well as the square of the nonlinear amplitude vanish.

In the following discussion representations in the $\left(X, k_{r}\right)$ - and the $\left(k_{r}, k_{i}\right)$ planes are always shown in parallel. Although the + and - superscripts may 
Fig. 13. (facing page) Evolution of CGL spatial branches with decreasing real frequency in a situation where the steep global mode is selected. The left sequence illustrates the evolution of the real part of linear branches $k_{r}^{\ell \pm}(X, \omega)$ (solid lines) and of nonlinear branches $k^{n \ell \pm}(X, \omega)$ (dashed lines) in the $(X, k)$-plane. Connecting points between linear and nonlinear branches are indicated with tick marks. In the right sequence, corresponding complex linear $k^{\ell \pm}(X, \omega)$ branches are sketched in the complex $\left(k_{r}, k_{i}\right)$-plane; superimposed on the same graphs are the curves $\left(k^{n \ell \pm}, R^{2}\right)$ of the nonlinear spatial branches. $(\mathrm{a}, \mathrm{b})$ For frequencies $\omega>\omega_{0}^{c a}$ linear spatial branches remain separated, nonlinear branches exist between the corresponding neutral stations. (c) For the front frequency $\omega_{0}^{c a}$, linear spatial branches pinch at $k_{0}^{c a}$ when $X=X^{c a}$. A sharp front associated with a wave number jump then gives rise to a global mode connecting the $k^{\ell-}$ branch near $X=-\infty$ to the $k^{\ell+}$ branch prevailing near $X=+\infty$. (d,e) For frequencies $\omega<\omega_{0}^{c a}$, branch switching between $k^{\ell+}$ and $k^{\ell-}$ occurs. (e) When $\omega=\omega_{s}^{n \ell}$, the nonlinear branches meet at the nonlinear saddle point location $X_{s}^{n \ell}$. However, this saddle point is not associated with a global mode since no continuous connection to the $k^{\ell-}$ branch prevailing near $X=-\infty$ exists.

not be derived from causality considerations in the AU domain, linear as well as the nonlinear + and - branches may be defined without ambiguity as long as the linear and nonlinear branches remain distinct (Sections 3.2 and 3.3).

The branch switching scenario as $\omega$ decreases depends on the relative values of the characteristic frequencies $\omega_{s}^{n \ell}$ and $\omega_{0}^{c a}$. The frequency $\omega_{0}^{a c}$ which corresponds to an unstable steep global mode with a front at the downstream boundary of the AU domain does not play an essential part. For clarity assume that $\omega_{0}^{c a}>\omega_{0}^{a c}$ and $\omega_{s}^{n \ell}>\omega_{0}^{a c}$. If these assumptions are not satisfied, the same selection mechanisms as discussed below prevail, although the detailed topology of spatial branches may be different. Two possibilities now arise: $\omega_{s}^{n \ell}>\omega_{0}^{c a}$ or $\omega_{s}^{n \ell}<\omega_{0}^{c a}$.

The scenario for $\omega_{s}^{n \ell}>\omega_{0}^{c a}$ is illustrated in the sequence of Fig.12. As $\omega$ is decreased, the two linear spatial branches successively cross the $k_{r}$-axis while remaining separated as depicted in Fig.12-a,b. Each crossing gives birth to a corresponding nonlinear branch, connected to its linear counterpart at the neutrally stable locations where $k^{\ell}$ is real. Both linear and nonlinear + and - branches are well identified and separated. When $\omega \downarrow \omega_{s}^{n \ell}$, the nonlinear branches gradually approach each other. For $\omega=\omega_{s}^{n \ell}$ (Fig.12-c), the $k^{n \ell+}$ branch meets the $k^{n \ell-}$ branch at $k_{s}^{n \ell}$ for $X=X_{s}^{n \ell}$ as determined by (30). This is the soft global mode configuration, where the nonlinear saddle point at $X_{s}^{n \ell}$ connects the nonlinear $k^{n \ell-}\left(X, \omega_{s}^{n \ell}\right)$ in the region $X<X_{s}^{n \ell}$ to the nonlinear $k^{n \ell+}\left(X, \omega_{s}^{n \ell}\right)$ in the region $X>X_{s}^{n \ell}$. Further outward, the nonlinear $k^{n \ell \pm}$ branches give way to their respective linear counterparts $k^{\ell \pm}$ at the locations of their respective neutral growth. When decreasing the global frequency to values $\omega<\omega_{s}^{n \ell}$, the nonlinear branches fail to exist in the neighborhood of $X_{s}^{n \ell}$ (Fig.12-d). As $\omega$ reaches $\omega_{0}^{c a}$ (Fig.12-e), linear spatial branches do pinch at $X=$ $X^{c a}$, but the nonlinear spatial branch $k^{n \ell+}$ prevailing around $X^{c a}$ cannot be 

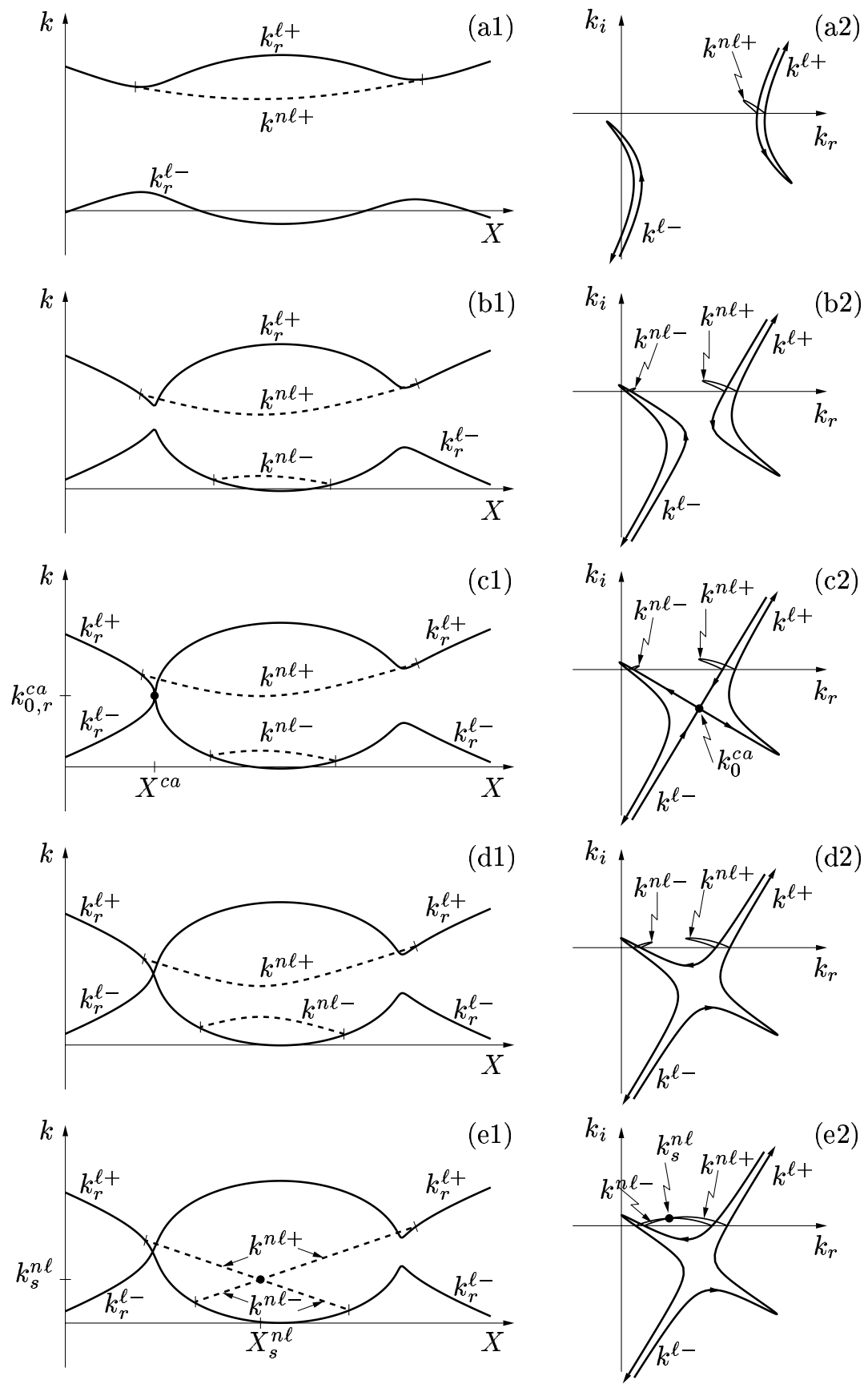

continued far downstream towards the $k^{\ell+}$ branch extending down to $X=$ $+\infty$ : There is no global mode of frequency $\omega_{0}^{c a}$.

The scenario for $\omega_{0}^{c a}>\omega_{s}^{n \ell}$ is sketched in the sequence of Fig.13. As $\omega$ decreases, the first characteristic frequency encountered is now $\omega_{0}^{c a}$. When $\omega \downarrow \omega_{0}^{c a}$ (Fig.13-a,b,c), the linear branches approach and pinch at $k_{0}^{c a}$ for $X=X^{c a}$ determined by (25). Since $k_{0, i}^{c a}<0$, the relevant branches in a neighborhood of $X^{c a}$ are $k^{\ell-}$ and $k^{n \ell+}$. A front of frequency $\omega_{0}^{c a}$ at $X^{c a}$ allows a discontinuous jump in wave number from $k^{\ell-}\left(X^{c a}, \omega_{0}^{c a}\right)=k_{0}^{c a}$ to $k^{n \ell+}\left(X^{c a}, \omega_{0}^{c a}\right)$. 
This front performs the connection between - and + branches necessary to obtain a steep global mode: further downstream, the amplitude of the nonlinear $k^{n \ell+}$ branch vanishes at a neutrally stable station and there the linear $k^{\ell+}$ branch takes over to $X=+\infty$. Note that the $k^{\ell+}\left(X, \omega_{0}^{c a}\right)$ branch necessarily crosses the $k_{r}$-axis; the $k^{\ell-}\left(X, \omega_{0}^{c a}\right)$ branch however may or may not cross. For $\omega=\omega_{0}^{c a}$, the nonlinear branches, if they both exist, do not meet. Decreasing further $\omega$ towards $\omega_{s}^{n \ell}$ (Fig.13-d,e), the nonlinear branches in turn approach and pinch for $\omega=\omega_{s}^{n \ell}$. However, since $\omega_{s}^{n \ell}<\omega_{0}^{c a}$, the linear $k^{\ell \pm}$ branches have undergone branch switching for $\omega=\omega_{0}^{c a}$. As can be seen in Fig.13-e, the nonlinear branches issuing at $k_{s}^{n \ell}, X_{s}^{n \ell}$ cannot be continued towards the $k^{\ell-}$ branch near $X=-\infty$. In this situation, due to global considerations, no soft mode exists, although the local criterion (30) may be satisfied.

This completes the discussion of the global mode selection mechanism: the type of selected global mode depends on the relative values of the linear pinchpoint frequency $\omega_{0}^{c a}$ and nonlinear pinchpoint frequency $\omega_{s}^{n \ell}$. If

$$
\omega_{s}^{n \ell}<\omega_{0}^{c a}
$$

linear spatial branches meet first as the overall frequency is lowered: the steep criterion (25) yields a global solution on the entire $X$-axis with a sharp front located at $X^{c a}$. However if

$$
\omega_{0}^{c a}<\omega_{s}^{n \ell}
$$

the nonlinear saddle point is encountered first and a soft global mode with overall smoothly varying envelope and wave number prevails. There exists of course situations where the nonlinear dispersion relation displays no saddle point in the nonlinear balloon. Then a steep mode is necessarily obtained.

The preceding close inspection of spatial linear and nonlinear branches guarantees that the selection criteria for steep and soft global modes are mutually exclusive, and that all situations are accounted for.

According to this discussion, when a global control parameter is continuously varied, the transition mechanism between soft and steep global modes is the following. Starting with a system where a soft mode is selected, i.e., where $\omega_{s}^{n \ell}>\omega_{0}^{c a}$, two possibilities arise if the control parameter is changed:

- The saddle point frequency $\omega_{s}^{n \ell}$ may equal the front frequency $\omega_{0}^{c a}$ for a critical value of the control parameter. Beyond this value, $\omega_{s}^{n \ell}<\omega_{0}^{c a}$, and a steep mode prevails. The transition to a steep mode of the same frequency takes place when $\omega_{s}^{n \ell}=\omega_{0}^{c a}$.

- The nonlinear saddle point may reach the boundary of the nonlinear balloon and disappear while still $\omega_{s}^{n \ell}>\omega_{0}^{c a}$. Then, transition to a steep mode again occurs, but in this situation the global frequency is discontinuous at transition. 
This will be fully justified in Section 4.9, where we map out the domains of existence of soft and steep global modes in an appropriate control parameter space. To identify the relevant control parameters, a discussion of the rôle of the absolute wave number is first required.

\subsection{Rôle of the absolute wave number}

The local instability properties are seen to be essentially controlled by the streamwise evolution of the absolute frequency $\omega_{0}(X)$ and wave number $k_{0}(X)$ which in principle can be varied independently. The criterion for steep global modes (25) only depends on the local absolute frequency $\omega_{0}(X)$ regardless of the local absolute wave number $k_{0}(X)$. The soft global mode criterion (30), however, depends on $k_{0}(X)$ through the complete nonlinear dispersion relation. In this subsection, the rôle of the absolute wave number is discussed by varying $k_{0}(X)$ in the CGL equation, all other coefficients remaining fixed.

From expressions (24) for the linear spatial $k^{\ell \pm}$ branches it is readily seen that a change in $k_{0}(X)$ by some constant value, say $\kappa$, results in a displacement of the $k^{\ell \pm}$ curves in the complex $k$-plane. Under this process, the linear pinchpoint properties are not affected. Nonlinear characteristics, however, are closely related to the crossing of the $k_{r}$-axis by the $k^{\ell \pm}$ branches; it follows that changes in $k_{0, i}$ strongly influence the nonlinear properties of the medium, unlike changes of $k_{0, r}$. This calls for two distinct physical interpretations of $k_{0, r}$ and $k_{0, i}$ respectively.

Consider the uniform CGL equation (6) with $k_{0}$ replaced by $k_{0}-\kappa_{r}$. Then, under the change of unknown function

$$
\psi(x, t)=\phi(x, t) e^{i \kappa_{r} x}
$$

the original CGL equation (6) is recovered for $\phi$. This shows that the only effect of a change in $k_{0, r}$ is a change in wave length; linear spatial growth or decay rates, frequencies as well as nonlinear amplitudes remain the same. A variation of $k_{0, r}$ results in a change of carrier wave but does not alter the linear nor the nonlinear stability properties of the system. In the spatially dependent CGL equation (4), the following change of unknown function

$$
\psi(x, t)=\phi(x, t) \exp \frac{i}{\epsilon} \int^{X} \kappa_{r}(u) d u
$$

results in modifying the absolute wave number to $k_{0}(X)-\kappa_{r}(X)$. Any slowly modulated carrier wave defined by the real function $\kappa_{r}(X)$ may thus be used to transform the real part of the absolute local wave number. Under such a transformation, the global mode selection criteria as well as the characteristic 
frequencies remain unaltered: The function $k_{0, r}(X)$ may be chosen arbitrarily since it does not affect the local and global dynamics.

In order to bring to the fore the rôle of $k_{0, i}(X)$, it is convenient, in the remainder of this section, to select $k_{0, r}(X)$ so that the function $\omega_{k k}(X) k_{0}(X)$ appearing in front of the advection term $\partial \psi / \partial x$ in (4) is real [12]. Under such a condition,

$$
k_{0, r}(X)=-\frac{\omega_{k k, r}(X)}{\omega_{k k, i}(X)} k_{0, i}(X),
$$

and the CGL equation reads

$$
\frac{\partial \psi}{\partial t}+\frac{\left|\omega_{k k}\right|^{2}}{\omega_{k k, i}} k_{0, i} \frac{\partial \psi}{\partial x}=-i\left(\omega_{0}+\frac{1}{2} \omega_{k k} k_{0}^{2}\right) \psi+\frac{i}{2} \omega_{k k} \frac{\partial^{2} \psi}{\partial x^{2}}-i \gamma|\psi|^{2} \psi
$$

The form (37) clearly indicates that the real factor

$$
U(X)=\frac{\left|\omega_{k k}(X)\right|^{2}}{\omega_{k k, i}(X)} k_{0, i}(X)
$$

may be interpreted as an advection velocity. Since causality requires that $\omega_{k k, i}<0$, a negative (positive) $k_{0, i}$ is readily seen to correspond to advection towards $X=+\infty(X=-\infty)$. Thus the sign of $k_{0, i}$ controls the advection direction whereas its magnitude is directly related to the advection velocity. As stated in the introduction, it is assumed that $U(X)>0$, i.e., $k_{0, i}(X)<0$ in the entire domain: the basic advection is then directed towards $X=+\infty$.

In order to further discuss the rôle of $k_{0, i}$ in the selection of global modes, consider, for definiteness, that $k_{0, i}$ is constant over the entire domain. A change in $k_{0, i}$ is seen to be associated with a displacement of the $k^{\ell \pm}$ curves along the $k_{i}$-axis in the complex $k$-plane. Its effect on the nonlinear balloon and nonlinear spatial branches in the $(X, k)$-plane is outlined in Fig.14. Since the function $\omega_{0}(X)$ is kept fixed, a change of $k_{0, i}$ leaves the extent of the AU range $X^{c a}<X<X^{a c}$ unaltered, as indicated by vertical dashed lines $X=X^{c a}$ and $X=X^{a c}$ in Fig.14. Without mean advection, $k_{0, i}=0$ (Fig.14-a), the nonlinear balloon exactly spans the AU region. In this situation, local linear instability coincides with local absolute instability: $X^{s c}=X^{c a}$ and $X^{a c}=X^{c s}$. Nonlinear spatial branches cover the nonlinear balloon symmetrically with respect to $k=k_{s}^{n \ell}$. Figures 14-b,c,d are obtained for increasing negative values of $k_{0, i}$, i.e., increasing advection towards $X=+\infty$. Increasing $\left|k_{0, i}\right|$ then shifts the $k^{\ell \pm}$ branches (24) towards negative $k_{i}$ in the complex $k$-plane, thereby enhancing the instability of the downstream $k^{\ell+}$ branches and reducing the instability of the upstream $k^{\ell-}$ branches. Under such circumstances, the onset of linear instability no longer coincides with transition to absolute instability: The nonlinear balloon inflates and extends beyond the AU range into the CU regions $X^{s c}<X<X^{c a}$ and $X^{a c}<X<X^{c s}$. The basic flow advection breaks the $k^{n \ell+} / k^{n \ell-}$ symmetry, and the part of the nonlinear balloon spanned 

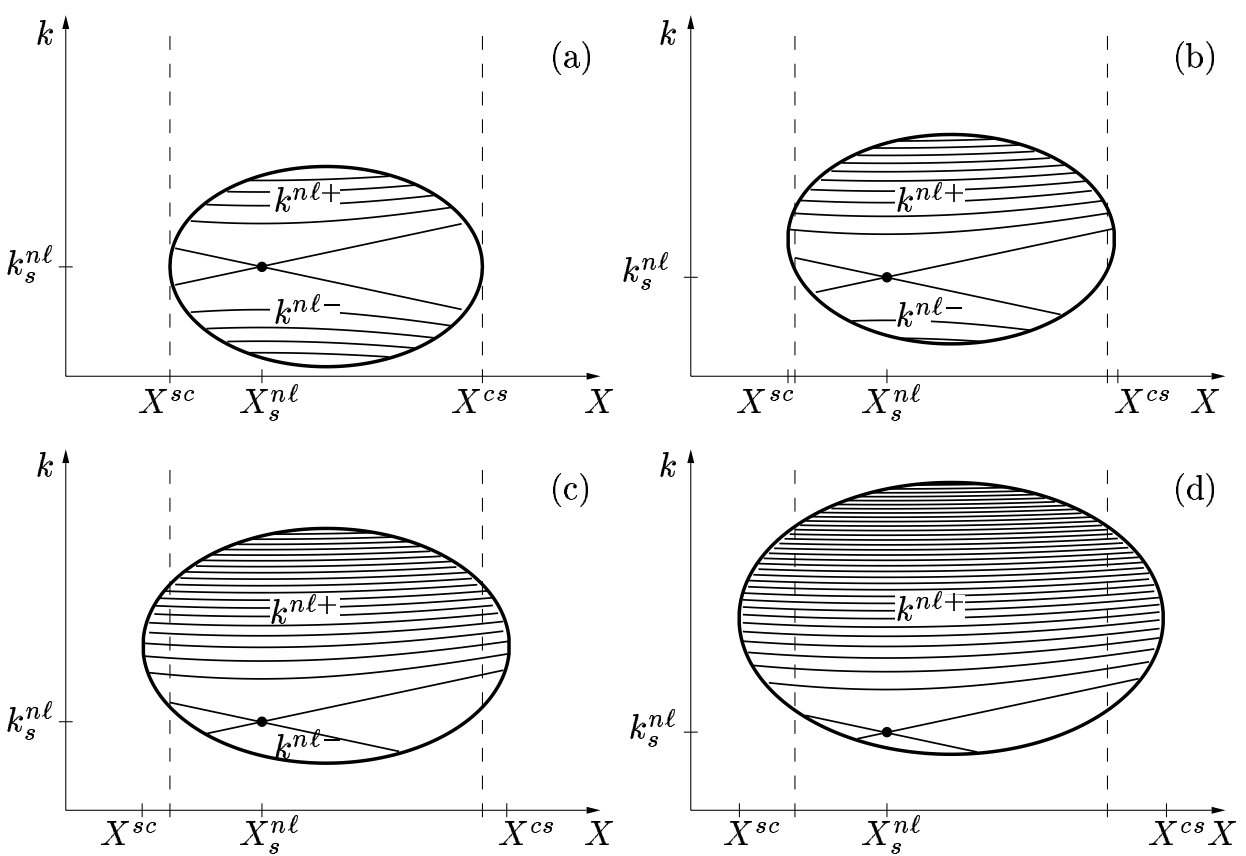

Fig. 14. Evolution of nonlinear balloon and spatial $k^{n \ell \pm}$ branches in the $(X, k)$-plane as the advection towards $X=+\infty$ is increased. Vertical dashed lines indicate AU domain extending over $X^{c a}<X<X^{a c}$. (a) Without mean advection $\left(k_{0, i}=0\right)$, spatial branches display symmetry with respect to the nonlinear saddle point wave number $k_{s}^{n \ell}$ and the nonlinear domain exactly spans the AU region, $X^{s c}=X^{c a}$ and $X^{a c}=X^{c s}$. (b,c,d) With increasing downstream advection $k_{0, i}<0$, the nonlinear balloon inflates and extends beyond the AU region, $X^{s c}<X^{c a}$ and $X^{a c}<X^{c s}$. During this process the fraction of the nonlinear balloon covered by the downstream $k^{n \ell+}$ branches increases with respect to the $k^{n \ell-}$ branches. Simultaneously, the nonlinear saddle point $\left(X_{s}^{n \ell}, k_{s}^{n \ell}\right)$ is seen to descend to eventually leave the nonlinear balloon.

by the $k^{n \ell+}$ branches increases to the detriment of the $k^{n \ell-}$ branches. During this process, the nonlinear saddle point moves towards the neutral stability boundary and eventually leaves the nonlinear balloon. The advection velocity, measured by $k_{0, i}$, thus strongly affects the existence of the nonlinear saddle point and hence the existence of smooth global modes.

In the remainder of this subsection we temporarily allow advection in the positive or negative direction so that $k_{0, i}$ may change sign. It has been shown in Section 4.2 that among the two stationary fronts at $X^{c a}$ and $X^{a c}$, only the one located at the upstream boundary of the AU region is stable. Since flow direction is directly related to the sign of $k_{0, i}$, the stable steep global mode frequency is $\omega_{0}^{c a}$ for $k_{0, i}<0$ and $\omega_{0}^{a c}$ for $k_{0, i}>0$. These are indeed the fronts at the stations of local marginal absolute instability with their nonlinear wave train covering the AU domain and their exponentially decaying tail extending into the CU region (see also Fig.15-a). The soft global mode frequency $\omega_{s}^{n \ell}$ has been obtained in (34), and, in contrast to the steep global mode frequencies $\omega_{0}^{c a}$ and $\omega_{0}^{a c}$, it is seen to depend on $k_{0, i}$. 


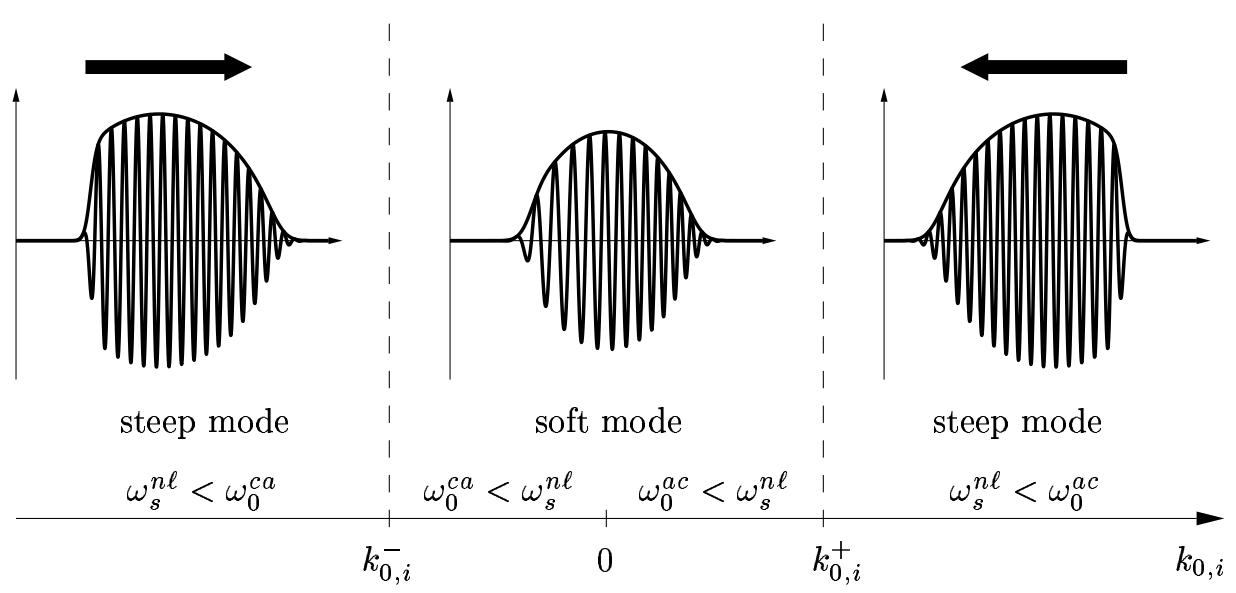

Fig. 15. Transition between steep and soft global modes as a function of $k_{0, i}$. Black arrows indicate advection direction. For small advection velocities $\left(k_{0, i}\right.$ small $)$, the saddle point frequency $\omega_{s}^{n \ell}$ is larger than the front frequencies $\omega_{0}^{c a}$ and $\omega_{0}^{a c}$, and thus a soft global mode is selected. With increasing advection towards $X=+\infty$ $\left(k_{0, i}<0\right)$, the saddle point frequency $\omega_{s}^{n \ell}$ decreases, and, when $k_{0, i}<k_{0, i}^{-}$the soft mode is replaced by a steep global mode with a front at the left boundary of the AU region. Similarly, with increasing advection towards $X=-\infty\left(k_{0, i}>0\right)$, the saddle point frequency $\omega_{s}^{n \ell}$ again decreases, and at $k_{0, i}=k_{0, i}^{+}$transition takes place to a steep global mode with a front at the right boundary of the AU region.

In the previous subsection it has been demonstrated that the global mode of largest frequency is selected; thus the transition scenario between steep and soft global modes as a function of $k_{0, i}$ is derived from the relative values of $\omega_{0}^{c a}, \omega_{0}^{a c}$ and $\omega_{s}^{n \ell}\left(k_{0, i}\right)$, as displayed in Fig.15. According to (34), the soft frequency $\omega_{s}^{n \ell}$ is largest for $k_{0, i}=0$, all other coefficients being kept fixed. Thus soft modes prevail when advection is small enough, $\omega_{s}^{n \ell}>\omega_{0}^{c a}$ and $\omega_{s}^{n \ell}>\omega_{0}^{a c}$. With increasing downstream advection $\left(k_{0, i}<0\right)$, the nonlinear saddle point frequency $\omega_{s}^{n \ell}$ decreases. When $\omega_{s}^{n \ell}<\omega_{0}^{c a}$, the soft mode is replaced by a steep mode with a sharp front at $X^{c a}$ (left-hand side of Fig.15). Similarly, if $k_{0, i}$ increases from $0\left(k_{0, i}>0\right)$, which corresponds to advection towards $X=-\infty$, the nonlinear saddle point frequency $\omega_{s}^{n \ell}$ again decreases. When $\omega_{s}^{n \ell}<\omega_{0}^{a c}$, the soft mode is now replaced by a steep mode with a front at $X=X^{a c}$ (righthand side of Fig.15). Since $k_{0, i}>0$, the front at $X^{a c}$ is now the stable one: upstream and downstream directions have been interchanged. In any case, the front location corresponds to the upstream boundary of the AU region. The critical transition values $k_{0, i}^{-}<0$ and $k_{0, i}^{+}>0$ between soft and steep global modes are defined by $\omega_{0}^{c a}=\omega_{s}^{n \ell}$ and $\omega_{0}^{a c}=\omega_{s}^{n \ell}$ respectively.

\subsection{Domains of existence in control parameter space}

Now that the rôles of $\omega_{0, i}^{\max }, \omega_{0 X, r}$ and $k_{0, i}$ have been separately discussed, the domains of existence of steep and soft global modes may be obtained in the three-dimensional space of these control parameters (Fig.16). Consider the 
CGL equation (4) with $\omega_{0}(X)$ of the form (28), all other coefficients being assumed uniform in $X$ for simplicity. The front frequencies $\omega_{0}^{c a}$ and $\omega_{0}^{a c}$ then read

$$
\omega_{0}^{c a}, \omega_{0}^{a c}=\omega_{0, r}^{\max }-\frac{\omega_{0 X X, r}}{\omega_{0 X X, i}} \omega_{0, i}^{\max } \mp \omega_{0 X, r} \sqrt{\frac{2 \omega_{0, i}^{\max }}{-\omega_{0 X X, i}}} .
$$

They exist whenever $\omega_{0, i}^{\max }>0$ and their values are seen to be effectively independent of $k_{0, i}$.

The selected gobal mode type depends on the relative values of the front frequencies (38) and the nonlinear saddle point frequency $\omega_{s}^{n \ell}$ obtained in eq.(34). However note that the nonlinear saddle point formally defined by $(32,33)$ only exists if $\left(k_{s}^{n \ell}, X_{s}^{n \ell}\right)$ lies in the nonlinear balloon, i.e., if

$$
\Omega_{i}^{\ell}\left(k_{s}^{n \ell}, X_{s}^{n \ell}\right)>0 \text {. }
$$

For the CGL equation under study, one readily obtains

$$
\begin{aligned}
\Omega_{i}^{\ell}\left(k_{s}^{n \ell}, X_{s}^{n \ell}\right)= & \omega_{0, i}^{\max }+\frac{1}{2} \omega_{0 X X, i}\left(\frac{\gamma_{i}}{\operatorname{Im} \gamma^{\star} \omega_{0 X X}}\right)^{2}\left(\omega_{0 X, r}\right)^{2} \\
& +\frac{\left|\omega_{k k}\right|^{2} \operatorname{Im} \gamma^{2} \omega_{k k}^{\star}}{2\left(\operatorname{Im} \gamma^{\star} \omega_{k k}\right)^{2}}\left(k_{0, i}\right)^{2}
\end{aligned}
$$

Whenever $\Omega_{i}^{\ell}\left(k_{s}^{n \ell}, X_{s}^{n \ell}\right)<0$, the nonlinear saddle point does not exist, and only a steep global mode is obtained. In the control parameter space of Fig.16, the domain where condition (39) is satisfied is located above the surface labelled $\Omega_{i}^{\ell}\left(k_{s}^{n \ell}, X_{s}^{n \ell}\right)=0$. According to (40), this surface is a paraboloid entirely contained in the half-space $\omega_{0, i}^{\max } \geq 0$ and tangent to the plane $\omega_{0, i}^{\max }=0$ at $\omega_{0 X, r}=k_{0, i}=0$. Below this surface no soft mode may exist and only steep modes are obtained. Above this surface, the soft global frequency $\omega_{s}^{n \ell}$ must be compared to $\omega_{0}^{c a}$ and $\omega_{0}^{a c}$. For simplicity consider only situations with advection towards $X=+\infty$, i.e., the half-space $k_{0, i}<0$, so that only $\omega_{0}^{c a}$ comes into consideration for steep modes. Within the region where a nonlinear saddle point exists, transition between soft and steep modes occurs when $\omega_{s}^{n \ell}=\omega_{0}^{c a}$. This transition surface is derived from (34) and (38) and it is sketched in Fig.16. It is seen to meet the surface $\Omega_{i}^{\ell}\left(k_{s}^{n \ell}, X_{s}^{n \ell}\right)=0$ along a curve in the plane $k_{0, i}=0$. As may be inferred from these critical surfaces, the parameter space is divided into four regions (Fig.16):

- (a) Below the global threshold, $\omega_{0, i}^{\max }<0$, no front and no saddle point exists, the unperturbed state $\psi=0$ remains stable.

- (b) When $\omega_{0, i}^{\max }>0$ and $\Omega_{i}^{\ell}\left(k_{s}^{n \ell}, X_{s}^{n \ell}\right)<0$, no saddle point exists and a steep global mode prevails.

- (c) When $\Omega_{i}^{\ell}\left(k_{s}^{n \ell}, X_{s}^{n \ell}\right)>0$ and $\omega_{s}^{n \ell}<\omega_{0}^{c a}$, a saddle point exists but the steep mode is selected. 


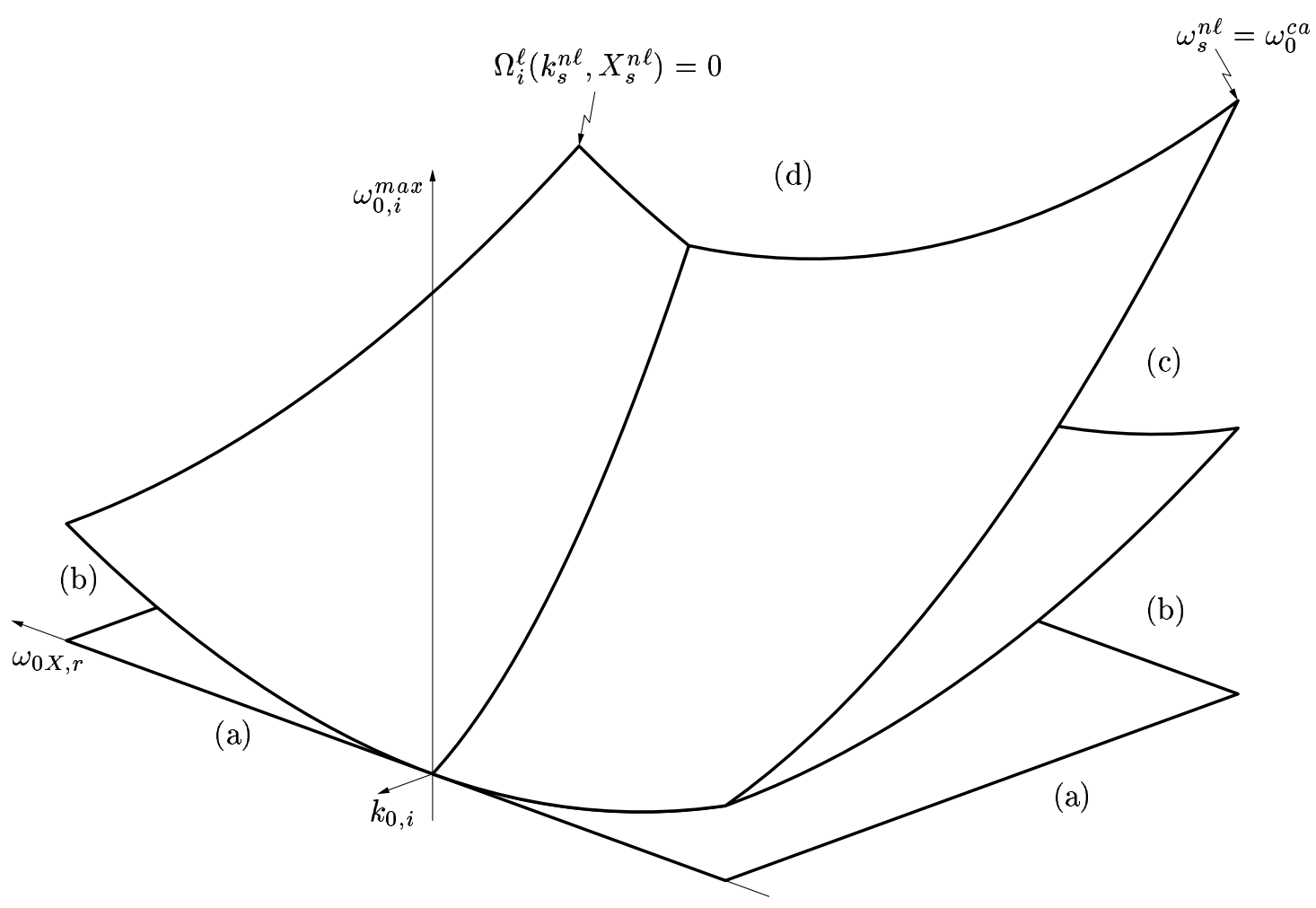

Fig. 16. Domains of existence of steep and soft global modes in the three-dimensional control parameter space $\left(\omega_{0 X, r}, k_{0, i}, \omega_{0, i}^{\text {max }}\right)$. A nonlinear saddle point exists above the surface $\Omega_{i}^{\ell}\left(k_{s}^{n \ell}, X_{s}^{n \ell}\right)=0$. The associated frequency $\omega_{s}^{n \ell}$ is larger than the front frequency $\omega_{0}^{c a}$ above the surface labelled $\omega_{s}^{n \ell}=\omega_{0}^{c a}$. Hence soft global modes prevail in region (d). In region (c), steep modes are selected according to $\omega_{s}^{n \ell}<\omega_{0}^{c a}$. In region (b), no nonlinear saddle point exists and only steep modes are obtained. In region (a), below global threshold $\omega_{0, i}^{\max }<0$, the unperturbed state is stable.

- (d) When $\Omega_{i}^{\ell}\left(k_{s}^{n \ell}, X_{s}^{n \ell}\right)>0$ and $\omega_{s}^{n \ell}>\omega_{0}^{c a}$, the soft mode is selected.

Thus at global mode onset $\omega_{0, i}^{\max }=0$, transition occurs always via a steep global mode except for the triply degenerate case $\omega_{0, i}^{\max }=k_{0, i}=\omega_{0 X, r}=0$. With increasing $\omega_{0, i}^{\max }$, transition from a steep to a soft mode occurs for finite values of $\omega_{0, i}^{\max }$ either as soon as the nonlinear saddle point comes into existence (on the surface $\Omega_{i}^{\ell}\left(k_{s}^{n \ell}, X_{s}^{n \ell}\right)=0$ ) or when its frequency reaches the steep frequency (on the surface $\omega_{s}^{n \ell}=\omega_{0}^{c a}$ ).

\subsection{Summary of transition mechanisms}

To conclude the first part of this paper, let us summarize the main transition mechanisms. Three distinct control parameters governing global mode selection have been identified.

The global bifurcation parameter is the maximum absolute growth rate over 
the entire medium $\omega_{0, i}^{\max }$. Nonlinear global modes exist whenever a region of absolute instability is present $\left(\omega_{0, i}^{\max }>0\right)$. The transition to a steep global mode occurs discontinuously at $\omega_{0, i}^{\max }=0$ through a saddle-node bifurcation. In the absence of absolute instability $\left(\omega_{0, i}^{\max }<0\right)$, no self-sustained global modes exist and the basic state is globally stable.

The basic state remains globally linearly stable up to a finite positive value of $\omega_{0, i}^{\max }$. The hysteresis width in $\omega_{0, i}^{\max }$ is governed by $\omega_{0 X, r}$ and scales as $\left(\omega_{0 X, r}\right)^{2}$.

The advection parameter $k_{0, i}$ strongly influences transition from steep to soft nonlinear global modes. Soft modes exist for small values of $k_{0, i}$, wheras for increasing upstream or downstream advection, steep modes prevail.

The main global mode properties may be inferred from the three above mentioned control parameters.

\section{$5 \quad$ Asymptotic spatial structure of global modes}

The preceding results have been derived under the assumption that the global mode is, at each station close to the local wave train at the global frequency. This local wave train is governed by the local linear dispersion relation (20) in regions where the amplitude is small, whereas it follows the local nonlinear dispersion relation (21) in finite amplitude domains. These considerations, which only involve the local characteristics of the medium, yield the leading order WKBJ approximation to the spatial structure. Within this framework, the selection criteria for steep and soft global modes have been identified and the leading order global frequencies have been derived.

The objective of this section is to incorporate the previous results into a consistent WKBJ approximation scheme [2], in order to obtain higher order correction terms, and to establish that the global mode structures outlined in the preceding sections may effectively be constructed by matching together extended wavepackets prevailing in different regions.

The organization of the following sections is motivated by the spatial structure of both steep and soft global modes illustrated in Fig.17. As already discussed, global modes display nonlinear regions of finite amplitude as well as linear regions of infinitesimal amplitude. In the outer semi-infinite linear regions $(\ell \pm$ ) extending towards $X= \pm \infty$ (Section 5.1) the respective complex linear spatial branch $k^{\ell \pm}$ prevails. The central nonlinear regions $(n \ell \pm)$ are of finite extent, i.e., order unity measured in terms of $X$, and they are dominated by the respective nonlinear spatial branch $k^{n \ell \pm}$ as discussed in Section 5.2. These extended regions are connected via three types of narrow transition layers: 

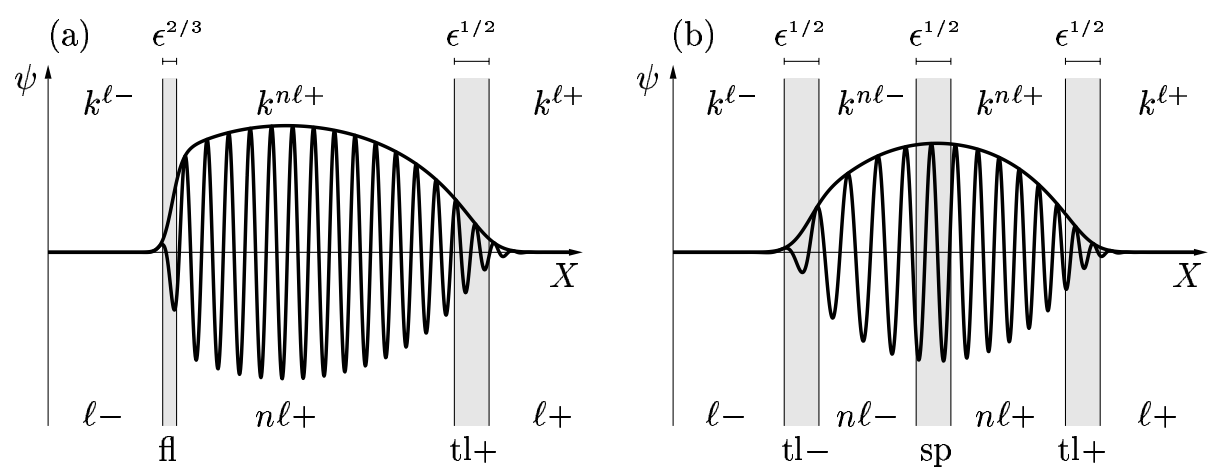

Fig. 17. Spatial structure of (a) steep and (b) soft global modes: $(\ell \pm)$, outer semi-infinite linear regions near $X= \pm \infty$ with respective linear spatial $k^{\ell \pm}$ branch; $(n \ell \pm)$, central nonlinear regions of size $\mathcal{O}(1)$ with respective nonlinear spatial $k^{n \ell \pm}$ branch; (tl \pm ), weakly nonlinear transition layers of width $\mathcal{O}\left(\epsilon^{1 / 2}\right)$ connecting linear and nonlinear branches of same superscript; (fl), front layer of size $\mathcal{O}\left(\epsilon^{2 / 3}\right)$ connecting the $k^{\ell-}$ and $k^{n \ell+}$ branches and selecting the steep global mode; (sp), saddle point layer of size $\mathcal{O}\left(\epsilon^{1 / 2}\right)$ connecting the $k^{n \ell \pm}$ branches and selecting the soft global mode.

- The front layer (fl) of size $\mathcal{O}\left(\epsilon^{2 / 3}\right)$ located at the upstream boundary of the steep global mode nonlinear region connects $k^{\ell-}$ and $k^{n \ell+}$ branches (Section 5.3).

- The nonlinear saddle point layer $(\mathrm{sp})$ of size $\mathcal{O}\left(\epsilon^{1 / 2}\right)$ allows crossover between the $k^{n \ell \pm}$ branches within the nonlinear soft global mode region (Section 5.4).

- Weakly nonlinear transition layers $(\mathrm{tl} \pm)$ of size $\mathcal{O}\left(\epsilon^{1 / 2}\right)$ at the downstream end of the steep global mode nonlinear region and at both ends of the soft global mode nonlinear region connect the fully developed nonlinear branches with their linear counterparts (Section 5.5).

Each of these regions is analysed in turn to obtain a uniformly valid asymptotic approximation over the entire range $-\infty<X<+\infty$. Since the width of the narrow layers is $\mathcal{O}\left(\epsilon^{1 / 2}\right)$ or $\mathcal{O}\left(\epsilon^{2 / 3}\right)$ measured in units of $X$, their characteristic scale is intermediate between the inhomogeneity length scale $\mathcal{O}(1)$ and the instability length scale $\mathcal{O}(\epsilon)$. Thus, although the medium may be considered uniform in the transition layers, they still display many wave lengths, typically $\mathcal{O}\left(\epsilon^{-1 / 2}\right)$ or $\mathcal{O}\left(\epsilon^{-1 / 3}\right)$

In the preceding sections, the bifurcation study was largely based on the CGL equation (4) and a complete understanding of the global selection mechanisms was achieved in this context. In the present section, the WKBJ approximation scheme is presented in the more general framework of p.d.e. (1). 


\subsection{Linear WKBJ instability waves}

In the semi-infinite linear regions extending towards $X= \pm \infty(\ell \pm$ in Fig.17$\mathrm{a}, \mathrm{b})$, the global mode amplitude exponentially decays on the fast $x$-scale. These regions are thus governed by the linear equation (2). Under the slowly varying medium hypothesis (3) a solution of (2) with global frequency $\omega_{g}$ may be obtained in terms of WKBJ approximations [2]. The spatial structure is described by a rapidly varying complex phase, accounting for the local wave length and spatial decay rate, and a slowly varying envelope. For a given value of the frequency $\omega_{g}$, the solution reads

$$
\psi(x, t)=A^{\ell}(X) \exp \left(\frac{i}{\epsilon} \int^{X} k^{\ell}(u) d u-i \omega_{g} t\right)+\text { c.c. }
$$

where $k^{\ell}(X)$ is one of the linear spatial branches associated with the frequency $\omega_{g}$. The functions $A^{\ell}(X)$ and $k^{\ell}(X)$ implicitly depend on $\omega_{g}$; for simplicity their frequency dependence is omitted.

In classical WKBJ fashion, the slowly varying envelope $A^{\ell}(X)$ is expanded in powers of $\epsilon$ as

$$
A^{\ell}(X) \sim A_{0}^{\ell}(X)+\epsilon A_{1}^{\ell}(X)+\epsilon^{2} A_{2}^{\ell}(X)+\ldots
$$

Thus, spatial differentiation takes the form

$$
\frac{\partial \psi}{\partial x}=\left[\left(i k^{\ell}(X)+\epsilon \partial_{X}\right) A^{\ell}(X)\right] \exp \left(\frac{i}{\epsilon} \int^{X} k^{\ell}(u) d u-i \omega_{g} t\right)+\text { c.c. }
$$

Upon substituting $(41,42,43)$ into the governing equation (2) and bearing in mind that $\Omega^{\ell}\left(-i \partial_{x}\right)=i \mathcal{L}\left(\partial_{x}\right)$, see $(8)$, one obtains

$$
\omega_{g}\left(A_{0}^{\ell}+\epsilon A_{1}^{\ell}+\ldots\right)=\Omega^{\ell}\left(k^{\ell}(X)-i \epsilon \partial_{X}, X\right)\left(A_{0}^{\ell}+\epsilon A_{1}^{\ell}+\ldots\right) .
$$

Note that differentiation with respect to the fast variable, i.e., multiplication by $i k^{\ell}(X)$, does not commute with the slow derivative operator $\partial_{X}$. The linear operator appearing in (44) admits the expansion

$$
\begin{aligned}
\Omega^{\ell}\left(k^{\ell}(X)-i \epsilon \partial_{X}, X\right) & =\Omega^{\ell}(X) \\
& -i \epsilon\left(\Omega_{k}^{\ell}(X) \partial_{X}+\frac{1}{2} k_{X}^{\ell}(X) \Omega_{k k}^{\ell}(X)\right)+\mathcal{O}\left(\epsilon^{2}\right),
\end{aligned}
$$

with the notations

$$
\Omega^{\ell}(X) \equiv \Omega^{\ell}\left(k^{\ell}(X), X\right), \quad \Omega_{k}^{\ell}(X) \equiv \frac{\partial \Omega^{\ell}}{\partial k}\left(k^{\ell}(X), X\right), \quad \text { etc. }
$$


At lowest order, eq.(44) reduces to the linear dispersion relation (20), i.e.,

$$
\omega_{g}=\Omega^{\ell}\left(k^{\ell}(X), X\right),
$$

which yields the local wave number $k^{\ell}(X)$ for a given frequency $\omega_{g}$.

The order $\epsilon$ terms read

$$
\left[\omega_{g}-\Omega^{\ell}\left(k^{\ell}(X), X\right)\right] A_{1}^{\ell}(X)=-i \Omega_{k}^{\ell}(X) \frac{d A_{0}^{\ell}}{d X}-\frac{i}{2} \Omega_{k k}^{\ell}(X) k_{X}^{\ell}(X) A_{0}^{\ell}(X) .
$$

By invoking (46), one obtains the obvious solvability condition for $A_{0}^{\ell}(X)$, namely

$$
\frac{d A_{0}^{\ell}}{d X}=-\frac{1}{2} k_{X}^{\ell}(X) \frac{\Omega_{k k}^{\ell}(X)}{\Omega_{k}^{\ell}(X)} A_{0}^{\ell}(X) .
$$

Thus, the first order asymptotic approximation to the solution of the linearized equation reads

$$
\begin{aligned}
& \psi \sim A_{0}^{\ell}\left(X_{1}\right) \exp \left(-\frac{1}{2} \int_{X_{1}}^{X} k_{X}^{\ell}(u) \frac{\Omega_{k k}^{\ell}(u)}{\Omega_{k}^{\ell}(u)} d u\right) \exp \left(\frac{i}{\epsilon} \int_{X_{1}}^{X} k^{\ell}(u) d u-i \omega_{g} t\right) \\
& \quad+\text { c.c. }
\end{aligned}
$$

where $X_{1}$ is some arbitrarily specified reference point. In each of the semiinfinite linear regions the respective causal $k^{\ell}$ branch has to be chosen: $k^{\ell-}$ for $X \rightarrow-\infty$ and $k^{\ell+}$ for $X \rightarrow+\infty$.

In the linear region, the nonlinear terms of (1) are seen to be exponentially smaller than the linear ones. The nonlinear operator then produces higher harmonics that are exponentially slaved to the fundamental (48). A complete expansion of $\psi$ reveals that the exponential envelope of the $n$-th harmonic reads

$$
\exp \left(-\frac{|n|}{\epsilon} \int^{X} k_{i}^{\ell}(u) d u\right)
$$

Their exact expressions are therefore irrelevant to this work and will not be computed here.

\subsection{Nonlinear WKBJ wave trains}

In the central nonlinear regions ( $n \ell \pm$ in Fig.17-a,b), the solution of (1) is obtained in terms of local nonlinear wavetrains (10). An asymptotic approximation scheme for nonlinear wavetrains is derived in this section, which is formally analogous to linear WKBJ theory. 
The fast oscillations of the propagating wave and its slowly varying local wave number and amplitude suggest the following change of variables,

$$
\psi(x, t)=\psi(\theta ; X)
$$

where the real phase function $\theta(x, t)$ is $2 \pi$-periodic and accounts for propagation on the fast space and time scales, whereas $X=\epsilon x$ allows for slow spatial modulation. Local frequency and wave number are defined as

$$
\omega=-\partial_{t} \theta \quad \text { and } \quad k=\partial_{x} \theta
$$

For a synchronized global solution, the frequency $\omega=\omega_{g}$ is constant, whereas the local wave number $k=k^{n \ell}(X)$ depends on the slow space variable.

Upon expanding the derivative operators according to

$$
\begin{aligned}
& \partial_{x}=k^{n \ell}(X) \partial_{\theta}+\epsilon \partial_{X} \\
& \partial_{t}=-\omega_{g} \partial_{\theta}
\end{aligned}
$$

the governing equation (1) is recast in the form

$$
\omega_{g} \partial_{\theta} \psi+\mathcal{F}\left(k^{n \ell}(X) \partial_{\theta}+\epsilon \partial_{X} ; X\right)[\psi]=0
$$

Again note that differentiation of $\psi(\theta ; X)$ with respect to the fast variable does not commute with $\partial_{X}$ since $k^{n \ell}(X)$ depends on $X$. Next $\psi$ is expanded according to

$$
\psi \sim \psi_{0}+\epsilon \psi_{1}+\epsilon^{2} \psi_{2}+\ldots,
$$

and substituted into (49).

The lowest order in $\epsilon$ yields the local equation

$$
\omega \partial_{\theta} \psi+\mathcal{F}\left(k \partial_{\theta} ; X\right)[\psi]=0
$$

where $X$ acts as an external parameter. When $X$ is considered frozen, the family of local nonlinear wavetrains $\Psi(\theta ; k, X)$ is recovered. This equation admits solutions only if $\omega=\Omega^{n \ell}(k, X)$. In other words, for a global frequency $\omega_{g}$, the nonlinear spatial branch $k^{n \ell}(X)$ is derived from the local nonlinear dispersion relation (21) as

$$
\omega_{g}=\Omega^{n \ell}\left(k^{n \ell}(X), X\right)
$$

and the leading order solution $\psi_{0}$ then reads

$$
\psi_{0}=\Psi\left(\theta ; k^{n \ell}(X), X\right) .
$$


The function $\Psi$ is $2 \pi$-periodic in the phase variable $\theta$ which accounts for the fast propagation through

$$
\theta=\frac{1}{\epsilon} \int^{X} k^{n \ell}(u) d u-\omega_{g} t+\theta_{0}(X)
$$

The so far undetermined slowly drifting phase function $\theta_{0}(X)$ obeys a solvability condition to be obtained at next order.

The $\mathcal{O}(\epsilon)$ terms in (49) require some care. For clarity of presentation assume that spatial differentiation only occurs in the linear operator,

$$
\mathcal{F}\left(\partial_{x} ; X\right)[\psi]=\mathcal{L}\left(\partial_{x} ; X\right)[\psi]+\mathcal{N}(X)[\psi]
$$

The linear terms are expanded as

$$
\begin{gathered}
\mathcal{L}\left(k^{n \ell}(X) \partial_{\theta}+\epsilon \partial_{X} ; X\right)\left[\psi_{0}(\theta ; X)+\epsilon \psi_{1}(\theta ; X)+\ldots\right] \sim \\
\mathcal{L}(X)\left[\psi_{0}\right]+\epsilon\left(\mathcal{L}(X)\left[\psi_{1}\right]+\mathcal{L}^{\prime}(X)\left[\partial_{X} \psi_{0}\right]+\frac{1}{2} \frac{d k^{n \ell}}{d X} \mathcal{L}^{\prime \prime}(X)\left[\partial_{\theta} \psi_{0}\right]\right)+\mathcal{O}\left(\epsilon^{2}\right),
\end{gathered}
$$

where the notation $\mathcal{L}(X)$ is shorthand for $\mathcal{L}\left(k^{n \ell}(X) \partial_{\theta} ; X\right)$ and the primes denote differentiation of $\mathcal{L}\left(\partial_{x} ; X\right)$ with respect to its first argument. The nonlinear terms are expanded as

$$
\mathcal{N}(X)\left[\psi_{0}+\epsilon \psi_{1}+\ldots\right]=\mathcal{N}(X)\left[\psi_{0}\right]+\epsilon \frac{\partial \mathcal{N}(X)\left[\psi_{0}\right]}{\partial \psi} \psi_{1}+\mathcal{O}\left(\epsilon^{2}\right)
$$

Thus (49) yields at $\mathcal{O}(\epsilon)$,

$$
L\left[\psi_{1}\right]=-\mathcal{L}^{\prime}(X)\left[\partial_{X} \psi_{0}\right]-\frac{1}{2} \frac{d k^{n \ell}}{d X} \mathcal{L}^{\prime \prime}(X)\left[\partial_{\theta} \psi_{0}\right]
$$

where the linear differential operator $L$ on the 1.h.s. is defined as

$$
L \equiv \omega_{g} \partial_{\theta}+\mathcal{L}(X)+\frac{\partial \mathcal{N}(X)\left[\psi_{0}\right]}{\partial \psi}
$$

Thus $\psi_{1}$ satisfies a linear differential equation with respect to $\theta$ with $X$ dependent coefficients. The operator $L$ is singular since one may readily verify that

$$
L\left[\Psi_{\theta}\right]=0 \quad \text { with } \quad \Psi_{\theta} \equiv \partial_{\theta} \Psi\left(\theta ; k^{n \ell}(X), X\right) .
$$

Thus (54) admits solutions for $\psi_{1}$ only if its r.h.s. satisfies a solvability condition.

Let us introduce an inner product for $2 \pi$-periodic functions of $\theta$ defined by

$$
\langle\phi, \psi\rangle=\frac{1}{2 \pi} \int_{0}^{2 \pi} \phi(\theta) \psi(\theta) d \theta .
$$


The adjoint operator $L^{\dagger}$ of $L$ is then obtained via successive integration by parts through the relation

$$
\langle\phi, L \psi\rangle=\left\langle L^{\dagger} \phi, \psi\right\rangle
$$

and it reads

$$
L^{\dagger}=-\omega_{g} \partial_{\theta}+\mathcal{L}\left(-k^{n \ell}(X) \partial_{\theta} ; X\right)+\frac{\partial \mathcal{N}(X)\left[\psi_{0}\right]}{\partial \psi} .
$$

Let $\Psi_{\theta}^{\dagger}$ be the adjoint eigenfunction of $\Psi_{\theta}$. Taking the inner product of (54) with $\Psi_{\theta}^{\dagger}$ and substituting

$$
\partial_{X} \psi_{0}=\frac{d \theta_{0}}{d X} \Psi_{\theta}+\frac{d k^{n \ell}}{d X} \Psi_{k}+\Psi_{X}
$$

yields the following solvability condition for $\theta_{0}(X)$,

$$
\frac{d \theta_{0}}{d X}\left\langle\Psi_{\theta}^{\dagger}, \mathcal{L}^{\prime} \Psi_{\theta}\right\rangle+\frac{1}{2} \frac{d k^{n \ell}}{d X}\left\langle\Psi_{\theta}^{\dagger}, 2 \mathcal{L}^{\prime} \Psi_{k}+\mathcal{L}^{\prime \prime} \Psi_{\theta}\right\rangle+\left\langle\Psi_{\theta}^{\dagger}, \mathcal{L}^{\prime} \Psi_{X}\right\rangle=0
$$

This entirely specifies the leading order approximation to the global nonlinear solution

$$
\psi(x, t) \sim \Psi\left(\frac{1}{\epsilon} \int^{X} k^{n \ell}(u) d u-\omega_{g} t+\theta_{0}(X) ; k^{n \ell}(X), X\right)
$$

where the parametric dependence of $k^{n \ell}(X)$ on the global frequency $\omega_{g}$ is entirely determined by (51).

\subsection{Front layer}

According to Section 4.2, the front of steep global modes is located at the station of local marginal absolute instability $X^{c a}$ and it is associated with a wave number jump from the $k^{\ell-}$ to the $k^{n \ell+}$ branch. On the slow scale $X$, the front discontinuously connects the linear solution (48) of wave number $k^{\ell-}$ prevailing in the upstream domain $X<X^{c a}$ to the nonlinear solution (59) of wave number $k^{n \ell+}$ on the downstream side of the front $X>X^{c a}$. In this subsection, the linear solution is shown to match to the nonlinear solution through a narrow front layer (fl) as depicted in Fig.17-a. The formulation essentially follows the same approach as in the asymptotic description of the front boundary layer arising in nonlinear dynamo waves developed by Bassom et al. [1] and is based on linear turning point theory [47].

The envelope of the outer linear solution is governed by the amplitude equation (47) which is singular at a turning point $X_{0}$ of the dispersion relation (46) 
defined by

$$
\omega_{0}\left(X_{0}\right)=\omega_{g}
$$

For $\omega_{g}=\omega_{0}^{c a}$, the turning point is at $X_{0}=X^{c a}$. However it is to be anticipated that the global frequency does not exactly equal $\omega_{0}^{c a}$. As outlined below, it is convenient to implement a matching procedure in the turning point region for an arbitrary complex global frequency $\omega_{g}$. Thus consider the linear governing equation (2) for complex $X$. The results of Section 5.1 pertaining to linear instability waves remain valid in the complex $X$-plane, provided that $\Omega^{\ell}(k, X)$ and hence $\omega_{0}(X)$ are analytically continued for complex values of $X$. Then (60) associates to a frequency $\omega_{g}$ the turning point $X_{0}\left(\omega_{g}\right)$ in the complex $X$-plane.

Expansion of the dispersion relation (46) in the neighborhood of the turning point $X_{0}$ yields

$$
0=\frac{1}{2} \Omega_{k k, 0}^{\ell}\left(k^{\ell}(X)-k_{0}\right)^{2}+\Omega_{X, 0}^{\ell}\left(X-X_{0}\right)+\text { h.o.t. }
$$

where $k_{0}$ is the associated absolute wavenumber $k_{0} \equiv k_{0}\left(X_{0}\right)$ and the subscript 0 denotes evaluation at $\left(k_{0}, X_{0}\right)$. By definition of the turning point, $\Omega_{k, 0}^{\ell}=0$, and under the assumption that the turning point is simple, $\Omega_{X, 0}^{\ell} \neq 0$. Thus

$$
k^{\ell}(X) \sim k_{0} \pm \sqrt{\frac{-2 \Omega_{X, 0}^{\ell}}{\Omega_{k k, 0}^{\ell}}}\left(X-X_{0}\right)^{1 / 2} .
$$

The linear amplitude equation (47) then reduces to

$$
\frac{d A_{0}}{d X} \sim-\frac{A_{0}(X)}{4\left(X-X_{0}\right)}
$$

Hence $A_{0}(X) \sim \mathrm{C}^{\text {st }}\left(X-X_{0}\right)^{-1 / 4}$ as $X \rightarrow X_{0}$, and the complete behavior of the outer linear solution (48) as $X \rightarrow X_{0}$ reads

$$
\psi \sim \mathrm{C}^{\mathrm{st}}\left(X-X_{0}\right)^{-1 / 4} \exp \left[\frac{2 i}{3 \epsilon} \sqrt{\frac{-2 \Omega_{X, 0}^{\ell}}{\Omega_{k k, 0}^{\ell}}\left(X-X_{0}\right)^{3}}\right] e^{i\left(k_{0} x-\omega_{g} t\right)}+\text { c.c. }
$$

Here it is assumed that the square root branch cut is chosen so that the wave number $k^{\ell-}$ prevailing in the domain $X \rightarrow-\infty$ is recovered.

It is seen that in the neighborhood of the turning point $X_{0}$, the amplitude $A_{0}$ becomes singular. Balance of dominant terms, as shown below, yields an inner turning point region of size $\mathcal{O}\left(\epsilon^{2 / 3}\right)$. Thus define an inner variable $\tilde{X}$ by

$$
X=X_{0}+\epsilon^{2 / 3} \tilde{X}
$$

and expand $\psi$ as

$$
\psi=\tilde{A}(\tilde{X}) e^{i\left(k_{0} x-\omega_{g} t\right)}+\text { c.c. },
$$


with

$$
\tilde{A}(\tilde{X})=\tilde{\mathrm{C}}^{\mathrm{st}}\left[\tilde{A}_{0}(\tilde{X})+\epsilon^{2 / 3} \tilde{A}_{2 / 3}(\tilde{X})+\ldots\right]
$$

where $\tilde{A}_{0}(\tilde{X})$ is $\mathcal{O}\left(\epsilon^{0}\right)$. Spatial differentiation now becomes

$$
\frac{\partial \psi}{\partial x}=\left[\left(i k_{0}+\epsilon^{1 / 3} \partial_{\tilde{X}}\right) \tilde{A}(\tilde{X})\right] e^{i\left(k_{0} x-\omega_{g} t\right)}+\text { c.c. }
$$

Note that in this inner transition layer, the leading order wave number is constant and equal to $k_{0}$, thus $\partial_{\tilde{X}}$ and $i k_{0}$ now commute. Replacing $(63,64,66)$ into the governing equation (2) yields

$$
\begin{aligned}
\omega_{g} \tilde{A}(\tilde{X}) & =\Omega^{\ell}\left(k_{0}-i \epsilon^{1 / 3} \partial_{\tilde{X}}, X_{0}+\epsilon^{2 / 3} \tilde{X}\right) \tilde{A}(\tilde{X}) \\
& \sim\left[\Omega^{\ell}\left(k_{0}, X_{0}\right)+\epsilon^{2 / 3}\left(\tilde{X} \Omega_{X, 0}^{\ell}-\frac{1}{2} \Omega_{k k, 0}^{\ell} \partial_{\tilde{X} \tilde{X}}\right)\right] \tilde{A}(\tilde{X})
\end{aligned}
$$

where $\omega_{g}=\Omega^{\ell}\left(k_{0}, X_{0}\right)$. The scaling $X-X_{0} \sim \epsilon^{2 / 3} \tilde{X}$ and $x \sim \epsilon^{-1 / 3} \tilde{X}$ guarantees the balance of dominant terms at order $\mathcal{O}\left(\epsilon^{2 / 3}\right)$ in the previous equation and leads to the following Airy equation for the leading order amplitude

$$
\frac{1}{2} \Omega_{k k, 0}^{\ell} \frac{d^{2} \tilde{A}_{0}}{d \tilde{X}^{2}}=\tilde{X} \Omega_{X, 0}^{\ell} \tilde{A}_{0}(\tilde{X})
$$

Thus $\tilde{A}_{0}(\tilde{X})$ is a linear superposition of the Airy functions $\operatorname{Ai}(-\lambda \tilde{X})$ and $\operatorname{Bi}(-\lambda \tilde{X})$ with

$$
\lambda^{3}=-\frac{2 \Omega_{X, 0}^{\ell}}{\Omega_{k k, 0}^{\ell}} .
$$

Upon choosing for $\lambda$ the solution with $|\operatorname{Arg} \lambda|<\pi / 3$, the Bi component exponentially grows whereas the $\mathrm{Ai}$ component decays according to

$$
\operatorname{Ai}(-\lambda \tilde{X}) \sim \frac{1}{2 \sqrt{\pi}}(-\lambda \tilde{X})^{-1 / 4} \exp \left[\frac{2 i}{3} \sqrt{\frac{-2 \Omega_{X, 0}^{\ell}}{\Omega_{k k, 0}^{\ell}} \tilde{X}^{3}}\right]
$$

as $\tilde{X} \rightarrow-\infty[2]$. Expressing the outer solution (62) in terms of the inner variable $\tilde{X}$, shows that it matches with the inner solution (64) provided that

$$
\tilde{A}(\tilde{X})=\tilde{\mathrm{C}}^{\mathrm{st}}\left[\operatorname{Ai}(-\lambda \tilde{X})+\mathcal{O}\left(\epsilon^{2 / 3}\right)\right]
$$

with $\mathrm{C}^{\text {st }}=\epsilon^{1 / 6} \frac{(-\lambda)^{-1 / 4}}{2 \sqrt{\pi}} \tilde{\mathrm{C}}^{\text {st }}$.

Thus, for any frequency $\omega_{g}$, linear instability waves are governed by the Airy equation (67) in a region of size $\mathcal{O}\left(\epsilon^{2 / 3}\right)$ located at the turning point of the linear local dispersion relation. The location of the turning point in the complex $X$-plane depends on $\omega_{g}$. For frequencies $\omega_{g}=\omega_{0}^{c a}+\delta \omega$, close to the marginal 
absolute frequency, the turning point is located at

$$
X_{0} \sim X^{c a}+\frac{\delta \omega}{\omega_{0, X}\left(X^{c a}\right)}
$$

For $\mathcal{O}\left(\epsilon^{2 / 3}\right)$ frequency corrections, $\delta \omega=\epsilon^{2 / 3} \omega_{2 / 3}$, the inner Airy region in the complex $X$-plane contains in its neighborhood the point $X^{c a}$ on the real $X$ axis.

Before analysing in more detail the properties of the Airy solutions on the $\tilde{X}$ scale, let us turn to the front structure. As discussed in Section 5.3, a uniform medium at the transition between convective and absolute instability admits stationary front solutions oscillating at its real absolute frequency. Thus the original nonlinear governing equation (1) rewritten at $X=X^{c a}$ as

$$
\omega_{0}^{c a} \frac{\partial \psi}{\partial \theta}+\mathcal{F}\left(\partial_{x} ; X^{c a}\right)[\psi]=0
$$

admits the front solution $\Psi_{f}(x, \theta)$, which is $2 \pi$-periodic in the phase function $\theta=\omega_{0}^{c a} t$. Towards $x=+\infty$ this solution approaches a fully nonlinear wavetrain of the form (52). Thus

$$
\Psi_{f}\left(x, \omega_{0}^{c a} t\right) \sim \Psi\left[k^{n \ell+}\left(X^{c a}, \omega_{0}^{c a}\right) x-\omega_{0}^{c a} t ; k^{n \ell+}\left(X^{c a}, \omega_{0}^{c a}\right), X^{c a}\right] \quad \text { as } \quad x \rightarrow+\infty .
$$

Towards $x=-\infty$ the exponential decay rate is dictated by the absolute wave number $k_{0}^{c a}=k_{0}\left(X^{c a}\right)$ and the front solution reads

$$
\Psi_{f}\left(x, \omega_{0}^{c a} t\right) \sim\left(c_{0}^{\mathrm{st}}+\mathrm{c}_{1}^{\mathrm{st}} x\right) e^{i\left(k_{0}^{c a} x-\omega_{0}^{c a} t\right)}+\text { c.c. },
$$

where the secular term is due to the double root $k^{\ell+}=k^{\ell-}=k_{0}^{c a}$ of the linear dispersion relation. In the uniform medium, the front has no preferred location. A translation of $\Delta x$ only changes the phase by $k^{n \ell+}\left(\omega_{0}^{c a}\right) \Delta x$ in the asymptotic behavior towards the nonlinear side $x=+\infty$ and leads to an additional factor $e^{i k_{0}^{c a} \Delta x}$ in the upstream exponentially decaying tail.

The exponentially decaying tail (71) has to be matched with (64) in the Airy region. Due to the secular term in (71), matching with (64) is only possible at the zeroes of the Airy function.

The Airy function Ai appearing in (69) admits real negative zeros $a_{i}$. Thus the zeros of $\tilde{A}_{0}$ are located at $\tilde{X}_{i}=-a_{i} / \lambda$. In terms of the outer variable $X$ these occur at

$$
X_{i} \equiv X_{0}\left(\omega_{g}\right)+\epsilon^{2 / 3} \tilde{X}_{i}=X^{c a}+\epsilon^{2 / 3}\left(\frac{\omega_{2 / 3}}{\omega_{0, X}}-\frac{a_{i}}{\lambda}\right)+\mathcal{O}\left(\epsilon^{4 / 3}\right) .
$$

Whereas the linear WKBJ approximations as well as the turning point region may be investigated in the complex $X$-plane, the front involves a nonlinear 
wavetrain and is thus necessarily restricted to the real $X$-axis. Requiring that the $i$-th zero (72) of the Airy function is on the real $X$-axis yields the frequency correction term

$$
\omega_{2 / 3}=a_{i} \frac{\left|\omega_{0, X}\right|^{2}}{|\lambda|^{2}} \frac{\operatorname{Im} \lambda}{\operatorname{Im} \omega_{0, X}}
$$

for a zero located at

$$
X_{i} \sim X^{c a}+\epsilon^{2 / 3} \frac{a_{i}}{|\lambda|^{2}} \frac{\operatorname{Im}\left(\omega_{0, X}^{\star} \lambda\right)}{\operatorname{Im} \omega_{0, X}}
$$

Since $X^{c a}$ is the transition location from convective to absolute instability, $\operatorname{Im} \omega_{0, X}$ is positive. Under the slightly more restrictive assumption that $\pi / 3<$ $\operatorname{Arg} \omega_{0, X}<2 \pi / 3$, we are guaranteed that $\operatorname{Im}\left(\omega_{0, X}^{\star} \lambda\right)<0$. In most situations of physical interest, variations of $\omega_{0, r}$ are small compared to those of $\omega_{0, i}$ and thus the condition on $\operatorname{Arg} \omega_{0, X}$ is readily fulfilled.

So far the matching conditions yield a countable set of frequencies

$$
\omega_{g} \sim \omega_{0}^{c a}+\epsilon^{2 / 3} a_{i} \Omega_{2 / 3} \quad \text { with } \quad \Omega_{2 / 3}=\frac{\left|\omega_{0, X}\right|^{2}}{|\lambda|^{2}} \frac{\operatorname{Im} \lambda}{\operatorname{Im} \omega_{0, X}}
$$

each being associated with the $i$-th zero $a_{i}<0$ of the Airy function. The corresponding fronts are located at

$$
X \sim X^{c a}-\epsilon^{2 / 3} a_{i} X_{2 / 3} \quad \text { with } \quad X_{2 / 3}=\frac{-\operatorname{Im}\left(\omega_{0, X}^{\star} \lambda\right)}{|\lambda|^{2} \operatorname{Im} \omega_{0, X}}>0
$$

Since $X_{2 / 3}>0$, higher order global modes display a front located further downstream in the AU domain. These situations are likely to be unstable since the exponentially decaying tail of the front partly penetrates into the AU domain. Such a higher order front prevailing in a slightly AU medium tends to move upstream, until it reaches the most upstream possible station associated with $a_{0}$. Thus we argue, although we have not proven the result, that the only stable global mode solution is obtained for the first zero indexed by $a_{0}$. Up to $\mathcal{O}\left(\epsilon^{2 / 3}\right)$, the global frequency and the front location therefore respectively read

$$
\omega_{g} \sim \omega_{0}^{c a}+\epsilon^{2 / 3} a_{0} \Omega_{2 / 3} \quad \text { and } \quad X \sim X^{c a}-\epsilon^{2 / 3} a_{0} X_{2 / 3}
$$

with

$$
a_{0}=-2.3381 \ldots
$$

This completes the investigation of the detailed structure of the front region. 


\subsection{Fully nonlinear saddle point layer}

The nonlinear saddle point is defined as the location $X_{s}^{n \ell}$ where the two nonlinear wave number branches meet. The saddle point frequency $\omega_{s}^{n \ell}$ is given by the criterion (30). In the nonlinear regions surrounding the saddle location $X_{s}^{n \ell}$ the asymptotic approximation of the global solution is of the form (59) and the slowly drifting phase function $\theta_{0}(X)$ is governed by the solvability condition (58).

At the saddle point, the first order differential equation (58) for $\theta_{0}$ becomes singular since $\left\langle\Psi_{\theta}^{\dagger}, \mathcal{L}^{\prime} \Psi_{\theta}\right\rangle=0$ at $X_{s}^{n \ell}$ [cf. equation (93) below]. Thus the nonlinear WKBJ approximation worked out in Section 5.2 is no longer valid in the neighborhood of $X_{s}^{n \ell}$, because $\theta_{0}$ varies there on a spatial scale which is faster than $X$. In this section, a nonlinear transition layer at $X_{s}^{n \ell}$ is introduced, (sp) in Fig.17-b, and a second order differential equation for the phase $\theta_{0}$ is derived after rescaling the spatial variable in the neighborhood of $X_{s}^{n \ell}$. This inner solution in the saddle point region allows a smooth crossover between the WKBJ wave trains in both nonlinear regions.

Let us introduce an inner local space variable $\tilde{X}$ in the saddle point region defined as

$$
X=X_{s}^{n \ell}+\epsilon^{1 / 2} \tilde{X}
$$

and expand $\psi$ according to

$$
\psi \sim \tilde{\psi}_{0}+\epsilon^{1 / 2} \tilde{\psi}_{1 / 2}+\epsilon \tilde{\psi}_{1}+\ldots
$$

The subsequent analysis yields the higher order corrections to the global frequency as

$$
\omega_{g} \sim \omega_{s}^{n \ell}+\epsilon^{1 / 2} \omega_{1 / 2}+\epsilon \omega_{1}+\ldots
$$

Replacing the spatial derivative $\partial_{x}$ in the inner region by $k_{s}^{n \ell} \partial_{\theta}+\epsilon^{1 / 2} \partial_{\tilde{X}}$, the linear operator expands as ${ }^{3}$

$$
\begin{aligned}
& \mathcal{L}\left(k_{s}^{n \ell} \partial_{\theta}+\epsilon^{1 / 2} \partial_{\tilde{X}}, X_{s}^{n \ell}+\epsilon^{1 / 2} \tilde{X}\right) \\
= & \mathcal{L}_{s}+\epsilon^{1 / 2}\left(\mathcal{L}_{s}^{\prime} \partial_{\tilde{X}}+\tilde{X} \mathcal{L}_{X, s}\right)+\frac{\epsilon}{2}\left(\mathcal{L}_{s}^{\prime \prime} \partial_{\tilde{X} \tilde{X}}+2 \tilde{X} \mathcal{L}_{X, s}^{\prime} \partial_{\tilde{X}}+\tilde{X}^{2} \mathcal{L}_{X X, s}\right) \\
& +\mathcal{O}\left(\epsilon^{3 / 2}\right) .
\end{aligned}
$$

Substituting the expansions $(75,76,77)$ into the governing equation (1), one recovers at leading order

$$
\omega_{s}^{n \ell} \partial_{\theta} \tilde{\psi}_{0}+\mathcal{L}\left(k_{s}^{n \ell} \partial_{\theta}, X_{s}^{n \ell}\right) \tilde{\psi}_{0}+\mathcal{N}\left(X_{s}^{n \ell}\right)\left[\tilde{\psi}_{0}\right]=0 .
$$

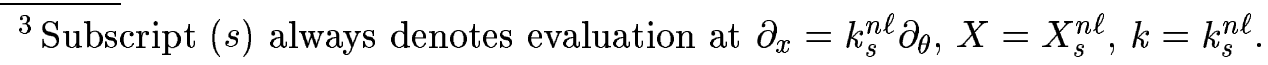


Thus

$$
\tilde{\psi}_{0}=\Psi_{s} \equiv \Psi\left(\theta+\tilde{\theta}_{0}(\tilde{X}) ; k_{s}^{n \ell}, X_{s}^{n \ell}\right)
$$

where the fast phase function in the inner saddle point layer reads $\theta=k_{s}^{n \ell} x-$ $\omega_{s}^{n \ell} t$ and the slow phase $\tilde{\theta}_{0}(\tilde{X})$ is a so far an undetermined function varying on the intermediate length scale $\tilde{X}$.

The nonlinear term is expanded as

$$
\begin{aligned}
& \mathcal{N}\left(X_{s}^{n \ell}+\epsilon^{1 / 2} \tilde{X}\right)\left[\Psi_{s}+\epsilon^{1 / 2} \tilde{\psi}_{1 / 2}+\epsilon \tilde{\psi}_{1}+\mathcal{O}\left(\epsilon^{3 / 2}\right)\right] \\
= & \mathcal{N}_{s}+\epsilon^{1 / 2}\left(\tilde{X} \mathcal{N}_{X, s}+\mathcal{N}_{\psi, s} \tilde{\psi}_{1 / 2}\right) \\
+ & \epsilon\left(\frac{1}{2} \tilde{X}^{2} \mathcal{N}_{X X, s}+\tilde{X} \mathcal{N}_{X \psi, s} \tilde{\psi}_{1 / 2}+\frac{1}{2} \mathcal{N}_{\psi \psi, s} \tilde{\psi}_{1 / 2}^{2}+\mathcal{N}_{\psi, s} \tilde{\psi}_{1}\right)+\mathcal{O}\left(\epsilon^{3 / 2}\right)
\end{aligned}
$$

where

$$
\mathcal{N}_{s} \equiv \mathcal{N}\left(X_{s}^{n \ell}\right)\left[\Psi_{s}\right], \quad \mathcal{N}_{\psi, s} \equiv \partial_{\psi} \mathcal{N}\left(X_{s}^{n \ell}\right)\left[\Psi_{s}\right], \quad \mathcal{N}_{X, s} \equiv \partial_{X} \mathcal{N}\left(X_{s}^{n \ell}\right)\left[\Psi_{s}\right], \quad \text { etc. }
$$

The order $\epsilon^{1 / 2}$ problem reads

$$
L_{s} \tilde{\psi}_{1 / 2}+\omega_{1 / 2} \Psi_{\theta, s}+\frac{d \tilde{\theta}_{0}}{d \tilde{X}} \mathcal{L}_{s}^{\prime} \Psi_{\theta, s}+\tilde{X}\left(\mathcal{L}_{X, s} \Psi_{s}+\mathcal{N}_{X, s}\right)=0
$$

where

$$
L_{s} \equiv \omega_{s}^{n \ell} \partial_{\theta}+\mathcal{L}_{s}+\mathcal{N}_{\psi, s}
$$

Let $\Psi_{\theta, s}^{\dagger}$ be the adjoint eigenfunction of $\Psi_{\theta, s}$. Eq. (80) admits solutions for $\tilde{\psi}_{1 / 2}$ if the following solvability condition is met

$$
\omega_{1 / 2}\left\langle\Psi_{\theta, s}^{\dagger}, \Psi_{\theta, s}\right\rangle+\frac{d \tilde{\theta}_{0}}{d \tilde{X}}\left\langle\Psi_{\theta, s}^{\dagger}, \mathcal{L}_{s}^{\prime} \Psi_{\theta, s}\right\rangle+\tilde{X}\left\langle\Psi_{\theta, s}^{\dagger}, \mathcal{L}_{X, s} \Psi_{s}+\mathcal{N}_{X, s}\right\rangle=0 .
$$

In Section 5.4.1 below, the two last terms of this equation are shown to vanish $(93,94)$. Thus the solvability condition requires that

$$
\omega_{1 / 2}=0
$$

There is no correction to the global frequency at order $\epsilon^{1 / 2}$ and no equation for $\tilde{\theta}_{0}$ is obtained at this order.

With $\omega_{1 / 2}=0$ and using $(95,96)$, the general solution of the linear inhomogenous equation (80) is obtained as

$$
\tilde{\psi}_{1 / 2}=\tilde{X} \Psi_{X, s}+\frac{d \tilde{\theta}_{0}}{d \tilde{X}} \Psi_{k, s}+A(\tilde{X}) \Psi_{\theta, s}
$$

where $A(\tilde{X})$ is an arbitrary real amplitude function. 
At order $\epsilon$, the following inhomogenous equation for $\tilde{\psi}_{1}$ is obtained

$$
\begin{aligned}
L_{s} \tilde{\psi}_{1} & +\mathcal{L}_{s}^{\prime} \partial_{\tilde{X}} \tilde{\psi}_{1 / 2}+\tilde{X}\left(\mathcal{L}_{X, s}+\mathcal{N}_{X \psi, s}\right) \tilde{\psi}_{1 / 2}+\frac{1}{2} \mathcal{N}_{\psi \psi, s} \tilde{\psi}_{1 / 2}^{2} \\
& +\omega_{1} \Psi_{s}+\left(\frac{1}{2} \mathcal{L}_{s}^{\prime \prime} \partial_{\tilde{X} \tilde{X}}+\tilde{X} \mathcal{L}_{\tilde{X}, s}^{\prime} \partial_{\tilde{X}}+\frac{1}{2} \tilde{X}^{2} \mathcal{L}_{X X, s}\right) \Psi_{s}+\frac{1}{2} \tilde{X}^{2} \mathcal{N}_{X X, s}=0
\end{aligned}
$$

Replacing $\tilde{\psi}_{1 / 2}$ according to (81) leads to

$$
\begin{aligned}
L_{s} \tilde{\psi}_{1}+ & \frac{1}{2} \frac{d^{2} \tilde{\theta}_{0}}{d \tilde{X}^{2}}\left(2 \mathcal{L}_{s}^{\prime} \Psi_{k, s}+\mathcal{L}_{s}^{\prime \prime} \Psi_{\theta, s}\right) \\
& +\frac{1}{2}\left(\frac{d \tilde{\theta}_{0}}{d \tilde{X}}\right)^{2}\left(2 \mathcal{L}_{s}^{\prime} \Psi_{\theta k, s}+\mathcal{L}_{s}^{\prime \prime} \Psi_{\theta \theta, s}+\mathcal{N}_{\psi \psi, s}\left(\Psi_{k, s}\right)^{2}\right) \\
& +\tilde{X} \frac{d \tilde{\theta}_{0}}{d \tilde{X}}\left(\left(\mathcal{L}_{X, s}+\mathcal{N}_{X \psi, s}\right) \Psi_{k, s}+\mathcal{L}_{X, s}^{\prime} \Psi_{\theta, s}+\mathcal{L}_{s}^{\prime} \Psi_{\theta X, s}+\mathcal{N}_{\psi \psi, s} \Psi_{k, s} \Psi_{X, s}\right) \\
& +\frac{1}{2} \tilde{X}^{2}\left(2\left(\mathcal{L}_{X, s}+\mathcal{N}_{X \psi, s}\right) \Psi_{X, s}+\mathcal{L}_{X X, s} \Psi_{s}+\mathcal{N}_{X X, s}+\mathcal{N}_{\psi \psi, s}\left(\Psi_{X, s}\right)^{2}\right) \\
& +\frac{d \tilde{\theta}}{d \tilde{X}} A(\tilde{X})\left(\mathcal{L}_{s}^{\prime} \Psi_{\theta \theta, s}+\mathcal{N}_{\psi \psi, s} \Psi_{\theta, s} \Psi_{k, s}\right) \\
& +\tilde{X} A(\tilde{X})\left(\left(\mathcal{L}_{X, s}+\mathcal{N}_{\psi X, s}\right) \Psi_{\theta, s}+\mathcal{N}_{\psi \psi, s} \Psi_{\theta, s} \Psi_{X, s}\right) \\
& +\frac{d A}{d \tilde{X}} \mathcal{L}_{s}^{\prime} \Psi_{\theta, s}+\frac{1}{2} A(\tilde{X})^{2} \mathcal{N}_{\psi \psi, s}\left(\Psi_{\theta, s}\right)^{2} \\
& +\mathcal{L}_{s}^{\prime} \Psi_{X, s}+\omega_{1} \Psi_{\theta, s} \\
& =0
\end{aligned}
$$

This equation admits solutions for $\tilde{\psi}_{1}$ if the inner product of the forcing terms (everything except $L_{s} \tilde{\psi}_{1}$ ) with $\Psi_{\theta, s}^{\dagger}$ vanishes. The different inner products are computed in Section 5.4.1. From $(97,98,99)$ it follows that all the terms involving the function $A(\tilde{X})$ vanish: this function remains undetermined at this order. Using $(100,101,102)$ the solvability condition for $\tilde{\theta}_{0}$ reads

$$
A \frac{d^{2} \tilde{\theta}_{0}}{d \tilde{X}^{2}}=B\left(\frac{d \tilde{\theta}_{0}}{d \tilde{X}}\right)^{2}+2 C \tilde{X} \frac{d \tilde{\theta}_{0}}{d \tilde{X}}+D \tilde{X}^{2}-E-2 \omega_{1}
$$

where

$$
\begin{aligned}
& A=\left\langle\Psi_{\theta, s}^{\dagger}, 2 \mathcal{L}_{s}^{\prime} \Psi_{k, s}+\mathcal{L}_{s}^{\prime \prime} \Psi_{\theta, s}\right\rangle /\left\langle\Psi_{\theta, s}^{\dagger}, \Psi_{\theta, s}\right\rangle \\
& B=\Omega_{k k, s}^{n \ell} \\
& C=\Omega_{k X, s}^{n \ell}, \\
& D=\Omega_{X X, s}^{n \ell} \\
& E=\left\langle\Psi_{\theta, s}^{\dagger}, \mathcal{L}_{s}^{\prime} \Psi_{X, s}\right\rangle /\left\langle\Psi_{\theta, s}^{\dagger}, \Psi_{\theta, s}\right\rangle
\end{aligned}
$$


Let

$$
\Theta(\tilde{X})=\exp \left[-\frac{B}{A} \tilde{\theta}_{0}(\alpha \tilde{X})-\frac{C \alpha^{2}}{2 A} \tilde{X}^{2}\right]
$$

with

$$
\alpha^{4}=\frac{A^{2}}{4\left(C^{2}-B D\right)} .
$$

Note that $C^{2}-B D>0$ since $\left(k_{s}^{n \ell}, X_{s}^{n \ell}\right)$ is a saddle point of $\Omega^{n \ell}(k, X)$. Rewriting the solvability condition (83) in terms of $\Theta$ yields the parabolic cylinder equation [2]

$$
\frac{d^{2} \Theta}{d \tilde{X}^{2}}=\left(\frac{1}{4} \tilde{X}^{2}-\nu-\frac{1}{2}\right) \Theta
$$

where

$$
\nu+\frac{1}{2}=\frac{1}{2} \frac{A C-B\left(E+2 \omega_{1}\right)}{\sqrt{A^{2}\left(C^{2}-B D\right)}} .
$$

This solution in the inner saddle point layer needs to be matched with the wave trains (59) in the outer nonlinear regions. In terms of the inner variable $\tilde{X}$, the phase of the outer nonlinear solutions (59) expands as

$$
\begin{aligned}
& \frac{1}{\epsilon} \int_{X_{s}^{n \ell}}^{X_{s}^{n \ell}+\epsilon^{1 / 2} \tilde{X}} k^{n \ell}(u) d u-\omega_{g} t+\theta_{0}(X) \\
& \quad=\left(k_{s}^{n \ell} x-\omega_{s}^{n \ell} t\right)+\frac{1}{2} k_{X, s}^{n \ell} \tilde{X}^{2}+\mathcal{O}\left(\epsilon^{1 / 2}\right)
\end{aligned}
$$

Matching to the phase $k_{s}^{n \ell} x-\omega_{s}^{n \ell} t+\tilde{\theta}_{0}(\tilde{X})$ of the inner solution (78) when $\tilde{X} \rightarrow \pm \infty$, requires that

$$
\tilde{\theta}_{0}(\tilde{X}) \sim \frac{1}{2} k_{X, s}^{n \ell} \tilde{X}^{2} \quad \text { when } \quad \tilde{X} \rightarrow \pm \infty
$$

At the saddle point, the derivative of the wave number reads

$$
k_{X, s}^{n \ell}=\frac{-\Omega_{k X, s}^{n \ell}+\sqrt{\left(\Omega_{k X, s}^{n \ell}\right)^{2}-\Omega_{X X, s}^{n \ell} \Omega_{k k, s}^{n \ell}}}{\Omega_{k k, s}^{n \ell}}=\frac{-C+\sqrt{C^{2}-B D}}{B} .
$$

Using (88) with (89) in (84) shows that the function $\Theta(\tilde{X})$ behaves asymptotically as

$$
\ln \Theta(\tilde{X}) \sim-\frac{1}{4} \tilde{X}^{2}
$$


when $\tilde{X} \rightarrow \pm \infty$. The only solution of (86) satisfying this asymptotic behavior and taking only nonnegative values is obtained for $\nu=0$ and simply reads

$$
\Theta(\tilde{X})=\exp \left(-\tilde{X}^{2} / 4\right)
$$

Thus the asymptotic expansion (88) is exact for all $\tilde{X}$. Then the soft global mode frequency reads to $\mathcal{O}(\epsilon)$

$$
\omega_{g} \sim \omega_{s}^{n \ell}+\epsilon \omega_{1}
$$

with

$$
\omega_{1}=\frac{1}{2 B}\left(A C-B E-\sqrt{A^{2}\left(C^{2}-B D\right)}\right) .
$$

\subsubsection{Computation of inner products}

The values of the inner products used in the previous analysis are obtained from

$$
\Omega^{n \ell}(k, X) \partial_{\theta} \Psi+\mathcal{L}\left(k \partial_{\theta}, X\right) \Psi+\mathcal{N}(X)[\Psi]=0
$$

Differentiation of (90) with respect to $\theta, k$ or $X$ yields

$$
\begin{array}{r}
L \Psi_{\theta} \equiv\left(\Omega^{n \ell} \partial_{\theta}+\mathcal{L}+\mathcal{N}_{\psi}\right) \Psi_{\theta}=0 \\
L \Psi_{k}+\Omega_{k}^{n \ell} \Psi_{\theta}+\mathcal{L}^{\prime} \Psi_{\theta}=0 \\
L \Psi_{X}+\Omega_{X}^{n \ell} \Psi_{\theta}+\mathcal{L}_{X} \Psi+\mathcal{N}_{X}=0 .
\end{array}
$$

Taking the inner product of $(91,92)$ with $\Psi_{\theta}^{\dagger}$ yields

$$
\begin{aligned}
\Omega_{k}^{n \ell}\left\langle\Psi_{\theta}^{\dagger}, \Psi_{\theta}\right\rangle+\left\langle\Psi_{\theta}^{\dagger}, \mathcal{L}^{\prime} \Psi_{\theta}\right\rangle & =0 \\
\Omega_{X}^{n \ell}\left\langle\Psi_{\theta}^{\dagger}, \Psi_{\theta}\right\rangle+\left\langle\Psi_{\theta}^{\dagger}, \mathcal{L}_{X} \Psi\right\rangle+\left\langle\Psi_{\theta}^{\dagger}, \mathcal{N}_{X}\right\rangle & =0 .
\end{aligned}
$$

At the saddle point $\left(k_{s}^{n \ell}, X_{s}^{n \ell}\right)$ the derivatives $\Omega_{k}^{n \ell}$ and $\Omega_{X}^{n \ell}$ vanish by definition, thus

$$
\begin{aligned}
\left\langle\Psi_{\theta, s}^{\dagger}, \mathcal{L}_{s}^{\prime} \Psi_{\theta, s}\right\rangle & =0, \\
\left\langle\Psi_{\theta, s}^{\dagger}, \mathcal{L}_{X, s} \Psi_{s}+\mathcal{N}_{X, s}\right\rangle & =0 .
\end{aligned}
$$

From (91) and (92) we deduce

$$
\begin{aligned}
L_{s} \Psi_{k, s} & =-\mathcal{L}_{s}^{\prime} \Psi_{\theta, s}, \\
L_{s} \Psi_{X, s} & =-\mathcal{L}_{X, s} \Psi_{\theta, s}-\mathcal{N}_{X, s} .
\end{aligned}
$$

From these identities, the particular solution (81) to eq.(80) follows. 
The order $\epsilon$ problem in the saddle point region specified by (82) requires to compute inner products by double differentiation. Differentiating (90) with respect to $\theta \theta, \theta k$ or $\theta X$ yields

$$
\begin{aligned}
L \Psi_{\theta \theta}+\mathcal{N}_{\psi \psi}\left(\Psi_{\theta}\right)^{2} & =0 \\
L \Psi_{\theta k}+\Omega_{k}^{n \ell} \Psi_{\theta \theta}+\mathcal{L}^{\prime} \Psi_{\theta \theta}+\mathcal{N}_{\psi \psi} \Psi_{\theta} \Psi_{k} & =0 \\
L \Psi_{\theta X}+\Omega_{X}^{n \ell} \Psi_{\theta \theta}+\mathcal{L}_{X} \Psi_{\theta}+\mathcal{N}_{X \psi} \Psi_{\theta}+\mathcal{N}_{\psi \psi} \Psi_{\theta} \Psi_{X} & =0
\end{aligned}
$$

and, after taking the inner product with $\Psi_{\theta}^{\dagger}$ at the saddle point, one obtains

$$
\begin{aligned}
\left\langle\Psi_{\theta, s}^{\dagger}, \mathcal{N}_{\psi \psi, s}\left(\Psi_{\theta, s}\right)^{2}\right\rangle & =0 \\
\left\langle\Psi_{\theta, s}^{\dagger}, \mathcal{L}_{s}^{\prime} \Psi_{\theta \theta, s}+\mathcal{N}_{\psi \psi, s} \Psi_{\theta, s} \Psi_{k, s}\right\rangle & =0 \\
\left\langle\Psi_{\theta, s}^{\dagger},\left(\mathcal{L}_{X, s}+\mathcal{N}_{X \psi, s}\right) \Psi_{\theta, s}+\mathcal{N}_{\psi \psi, s} \Psi_{\theta, s} \Psi_{X, s}\right\rangle & =0 .
\end{aligned}
$$

Differentiation of (90) with respect to $k k, k X$ or $X X$ yields

$$
\begin{aligned}
& L \Psi_{k k}+\Omega_{k k}^{n \ell} \Psi_{\theta}+2\left(\Omega_{k}^{n \ell}+\mathcal{L}^{\prime}\right) \Psi_{\theta k}+\mathcal{L}^{\prime \prime} \Psi_{\theta \theta}+\mathcal{N}_{\psi \psi}\left(\Psi_{k}\right)^{2}=0, \\
& L \Psi_{k X}+\left(\Omega_{k X}^{n \ell}+\mathcal{L}_{X}^{\prime}\right) \Psi_{\theta}+\left(\Omega_{k}^{n \ell}+\mathcal{L}^{\prime}\right) \Psi_{\theta X} \\
& +\Omega_{X}^{n \ell} \Psi_{\theta k}+\left(\mathcal{L}_{X}+\mathcal{N}_{X \psi}\right) \Psi_{k}+\mathcal{N}_{\psi \psi} \Psi_{k} \Psi_{X}=0, \\
& L \Psi_{X X}+\Omega_{X X}^{n \ell} \Psi_{\theta}+2 \Omega_{X}^{n \ell} \Psi_{\theta X}+2\left(\mathcal{L}_{X}+\mathcal{N}_{X \psi}\right) \Psi_{X} \\
& +\mathcal{L}_{X X} \Psi+\mathcal{N}_{\psi \psi}\left(\Psi_{X}\right)^{2}+\mathcal{N}_{X X}=0,
\end{aligned}
$$

and thus

$$
\begin{array}{r}
\left\langle\Psi_{\theta, s}^{\dagger}, 2 \mathcal{L}_{s}^{\prime} \Psi_{\theta k, s}+\mathcal{L}_{s}^{\prime \prime} \Psi_{\theta \theta, s}+\mathcal{N}_{\psi \psi, s}\left(\Psi_{k, s}\right)^{2}\right\rangle \\
=-\Omega_{k k, s}^{n \ell}\left\langle\Psi_{\theta, s}^{\dagger}, \Psi_{\theta, s}\right\rangle \\
\left\langle\Psi_{\theta, s}^{\dagger},\left(\mathcal{L}_{X, s}+\mathcal{N}_{X \psi, s}\right) \Psi_{k, s}+\mathcal{L}_{X, s}^{\prime} \Psi_{\theta, s}+\mathcal{L}_{s}^{\prime} \Psi_{\theta X, s}+\mathcal{N}_{\psi \psi, s} \Psi_{k, s} \Psi_{X, s}\right\rangle \\
=-\Omega_{k X, s}^{n \ell}\left\langle\Psi_{\theta, s}^{\dagger}, \Psi_{\theta, s}\right\rangle \\
\left\langle\Psi_{\theta, s}^{\dagger}, 2\left(\mathcal{L}_{X, s}+\mathcal{N}_{X \psi, s}\right) \Psi_{X, s}+\mathcal{L}_{X X, s} \Psi_{s}+\mathcal{N}_{X X, s}+\mathcal{N}_{\psi \psi, s}\left(\Psi_{X, s}\right)^{2}\right\rangle \\
=-\Omega_{X X, s}^{n \ell}\left\langle\Psi_{\theta, s}^{\dagger}, \Psi_{\theta, s}\right\rangle
\end{array}
$$

Results (97-102) are invoked to cast the phase evolution equation in its final form (83).

\subsection{Weakly nonlinear transition layers}

Frequency corrections for steep and soft global modes have been derived by performing a detailed asymptotic analysis of the corresponding narrow transition layers where their respective frequency selection mechanism takes place: the front layer for steep modes and the nonlinear saddle point layer for soft modes. The only type of layer that remains to be investigated in order to 
obtain uniformly valid asymptotic approximations for global modes is the weakly nonlinear transition layer. This layer applies to the downstream end of the steep mode nonlinear region (tl+ in Fig.17-a) and to both ends of the soft mode nonlinear region (tl \pm in Fig.17-b). It should be emphasized that these layers are slaved to the dynamics imposed by the front or the nonlinear saddle point frequency.

A smooth transition between nonlinear and linear solutions occurs at the boundary of the nonlinear balloon (Fig.5 and 7) in the $(X, k)$-plane. Consider a global solution of frequency $\omega_{g}$. Its local linear and nonlinear wave number branches $k^{\ell}\left(X, \omega_{g}\right)$ and $k^{n \ell}\left(X, \omega_{g}\right)$ are derived respectively from the curves $\Omega^{\ell}(k, X)=\omega_{g}$ and $\Omega^{n \ell}(k, X)=\omega_{g}$. A $k^{n \ell}\left(X, \omega_{g}\right)$ branch is connected at the border of the nonlinear balloon to the corresponding linear $k^{\ell}\left(X, \omega_{g}\right)$ branch at the particular station $X=X_{t}\left(\omega_{g}\right)$ for $k=k_{t}\left(\omega_{g}\right)$ where the pair $\left(k_{t}, X_{t}\right)$ is defined by

$$
\omega_{g}=\Omega^{\ell}\left(k_{t}, X_{t}\right)=\Omega^{n \ell}\left(k_{t}, X_{t}\right)
$$

As demonstrated in Sections 4.2 and 4.6, a continuous transition between linear and nonlinear wave number branches occurs at the downstream boundary of the nonlinear region of a steep global mode and at both downstream and upstream boundaries of the nonlinear region of a soft global mode. At a downstream boundary, the $k^{n \ell+}$ branch prevailing in the region $X<X_{t}$ is continuously connected to the $k^{\ell+}$ branch for $X>X_{t}$. At an upstream boundary, transition from $k^{\ell-}$ for $X<X_{t}$ to $k^{n \ell-}$ for $X>X_{t}$ occurs.

When $X \rightarrow X_{t}$ from within the nonlinear region, the amplitude of the nonlinear wave train decays, nonlinearities weaken, higher harmonics become slaved to the fundamental, and eventually a linear instability wave takes over. In the present section we show how the connection between linear and nonlinear solutions is achieved across a narrow transition layer located at $X_{t}$.

Let us focus on an upstream transition layer (tl-), i.e., the solution is fully nonlinear for $X>X_{t}$ and decays exponentially for $X<X_{t}$. The same analysis applies in the downstream transition layer $(\mathrm{tl}+)$. The asymptotic behavior of the nonlinear wave trains $\Psi(\theta ; k, X)$ is first derived as $(k, X)$ approaches the neutral stability boundary of the nonlinear balloon (Section 5.5.1). This result is then used along the particular path $\left(k^{n \ell}(X), X\right)$ to derive expansions for the modulus (Section 5.5.2) and phase (Section 5.5.3) of the global mode as $X \downarrow X_{t}$. This outer expansion is shown in Section 5.5.4 to match with the inner solution prevailing in the transition layer. Finally the inner solution is matched in Section 5.5.5 to the outer linear WKBJ approximation prevailing in the region $X<X_{t}$. 


\subsubsection{Weakly nonlinear behavior}

In the nonlinear balloon of the $(X, k)$-plane characterized by $\Omega_{i}^{\ell}(k, X)>0$, the governing equation (1) admits local solutions of the form (52). A global mode of frequency $\omega_{g}$ follows the line $\omega_{g}=\Omega^{n \ell}(k, X)$ in the $(k, X)$-plane. The boundary of the nonlinear balloon is reached at $\left(k_{t}, X_{t}\right)$ defined in (103). Let us first study the behavior of the periodic functions $\Psi(\theta ; k, X)$ in the $(k, X)$-plane as $(k, X)$ approaches the boundary of the nonlinear balloon, i.e., $\Omega_{i}^{\ell}(k, X) \downarrow 0$.

The nonlinear operator $\mathcal{F}[\psi]$ in (1) is expanded in powers of $\psi$ and (1) is rewritten as

$$
\frac{\partial \psi}{\partial t}=\mathcal{L}\left(\partial_{x}, X\right)[\psi]+\sum_{r=2}^{\infty} \mathcal{N}_{r}\left(\partial_{x}, X\right)[\psi]
$$

where the operators $\mathcal{N}_{r}$ are of $r$-th order in $\psi$. In all generality, each $\mathcal{N}_{r}$ depends on $\partial_{x}$, but to simplify the subsequent computations, it is assumed that the $\mathcal{N}_{r}$ 's do not involve spatial derivation, i.e.,

$$
\mathcal{N}_{r}\left(\partial_{x}, X\right)[\psi]=N_{r}(X) \psi^{r}
$$

The results would remain valid for any nonlinear operator with spatial derivatives, but the notation and results becom unwieldy in more general cases. The method is easily extended to specific examples.

The $2 \pi$-periodic function $\Psi$ is expanded as the Fourier series

$$
\Psi(\theta ; k, X)=\sum_{n} \Psi^{(n)}(k, X) e^{i n \theta}
$$

where $\Psi^{(-n)}=\left(\Psi^{(n)}\right)^{\star}$. Due to the invariance of the governing equation under the transformation $\theta \rightarrow \theta+\mathrm{C}^{\text {st }} ., \Psi^{(1)}(k, X)$ may be chosen to be real for each $(k, X)$.

Substituting $(105,106)$ into $(104)$ yields the equations for the harmonic components of $\Psi$,

$$
\begin{aligned}
n \Omega^{n \ell}(k, X) \Psi^{(n)}(k, X)= & \Omega^{\ell}(n k, X) \Psi^{(n)}(k, X) \\
& +i \sum_{r=2}^{\infty} N_{r}(X) \sum_{n_{1}+\ldots+n_{r}=n} \Psi^{\left(n_{1}\right)}(k, X) \cdots \Psi^{\left(n_{r}\right)}(k, X) .
\end{aligned}
$$

With the definition

$$
\Delta^{(n)}(k, X)=n \Omega^{n \ell}(k, X)-\Omega^{\ell}(n k, X),
$$

equations (107) read, 


$$
\begin{aligned}
& \Delta^{(n)}(k, X) \Psi^{(n)}(k, X) \\
= & i \sum_{r=2}^{\infty} N_{r}(X) \sum_{n_{1}+\ldots+n_{r}=n} \Psi^{\left(n_{1}\right)}(k, X) \cdots \Psi^{\left(n_{r}\right)}(k, X) .
\end{aligned}
$$

When $\Omega_{i}^{\ell}(k, X) \downarrow 0$, the term $\Delta^{(1)}(k, X)$ vanishes, whereas the $\Delta^{(n)}(k, X)$ remain finite for $|n| \neq 1$. Thus, in this limit, the components with $|n| \neq 1$ are slaved to the fundamental $n= \pm 1$ and scale as

$$
\Psi^{(n)}(k, X)=\left\{\begin{array}{l}
\mathcal{O}\left[\left(\Psi^{(1)}(k, X)\right)^{|n|}\right] \text { if } n \neq 0 \\
\mathcal{O}\left[\left(\Psi^{(1)}(k, X)\right)^{2}\right] \text { if } n=0
\end{array} \quad \text { when } \quad \Omega_{i}^{\ell}(k, X) \downarrow 0\right.
$$

The dominant terms of (108) for $n=2 \mathrm{read}$

$$
\Delta^{(2)}(k, X) \Psi^{(2)}(k, X)=i N_{2}(X)\left[\Psi^{(1)}(k, X)\right]^{2}+\mathcal{O}\left[\left(\Psi^{(1)}\right)^{4}\right]
$$

and for $n=0$,

$$
\Delta^{(0)}(k, X) \Psi^{(0)}(k, X)=2 i N_{2}(X) \Psi^{(1)}(k, X) \Psi^{(-1)}(k, X)+\mathcal{O}\left[\left(\Psi^{(1)}\right)^{4}\right] .
$$

For $n=1$, equation (108) yields

$$
\begin{aligned}
& \Delta^{(1)}(k, X) \Psi^{(1)}(k, X)=2 i N_{2}(X)\left[\Psi^{(2)}(k, X) \Psi^{(-1)}(k, X)+\Psi^{(1)}(k, X) \Psi^{(0)}(k, X)\right] \\
& \quad+3 i N_{3}(X)\left|\Psi^{(1)}(k, X)\right|^{2} \Psi^{(1)}(k, X)+\mathcal{O}\left[\left(\Psi^{(1)}\right)^{5}\right]
\end{aligned}
$$

Substituting $(110,111)$ into $(112)$ yields the leading order expression for $\Psi^{(1)}$

$$
\left|\Psi^{(1)}(k, X)\right|^{2} \sim \frac{\Delta^{(1)}(k, X)}{3 i N_{3}(X)-2 N_{2}(X)^{2}\left(\frac{1}{\Delta^{(2)}(k, X)}+\frac{2}{\Delta^{(0)}(k, X)}\right)} .
$$

Thus

$$
\left|\Psi^{(1)}(k, X)\right|^{2}=\mathcal{O}\left[\Delta^{(1)}(k, X)\right]
$$

and

$$
\Psi^{(1)}(k, X)=\mathcal{O}\left[\sqrt{\Omega_{i}^{\ell}(k, X)}\right]
$$

\subsubsection{Asymptotic decay of outer nonlinear wave trains}

The preceding results, valid for any $(k, X)$ when $\Omega_{i}^{\ell}(k, X) \downarrow 0$, yield the asymptotic behavior of the nonlinear part (59) of a global mode as $X \downarrow X_{t}$.

Let us expand the nonlinear solution (59) into harmonic components as follows 


$$
\begin{aligned}
& \Psi\left(\theta ; k^{n \ell}(X), X\right)= \\
& \sum_{n}\left(\psi_{0}^{(n)}(X)+\epsilon \psi_{1}^{(n)}(X)+\ldots\right) \exp \left\{i n\left(\frac{1}{\epsilon} \int^{X} k^{n \ell}(u) d u-\omega_{g} t\right)\right\} .
\end{aligned}
$$

Then, according to (106),

$$
\psi_{0}^{(n)}(X)=\Psi^{(n)}\left(k^{n \ell}(X), X\right) e^{i n \theta_{0}(X)} .
$$

From $(109,114)$ with $\Omega_{i}^{\ell}\left(k^{n \ell}(X), X\right)=\mathcal{O}\left(X-X_{t}\right)$, it follows that, for each $n \neq 0$,

$$
\psi_{0}^{(n)}(X)=\mathcal{O}\left[\left(X-X_{t}\right)^{|n| / 2}\right] \quad \text { as } \quad X \downarrow X_{t} .
$$

The asymptotic behavior of $\psi_{0}^{(1)}(X)$ for $X \downarrow X_{t}$ is derived from (113) as

$$
\psi_{0}^{(1)}(X) \sim \hat{\Psi}_{0}^{(1)} e^{i \theta_{0}(X)}\left(X-X_{t}\right)^{1 / 2},
$$

with

$$
\left|\hat{\Psi}_{0}^{(1)}\right|^{2}=\frac{\Delta_{X, t}^{(1)}}{3 i N_{3, t}-2\left(N_{2, t}\right)^{2}\left(\frac{1}{\Delta_{t}^{(2)}}+\frac{2}{\Delta_{t}^{(0)}}\right)},
$$

where subscript $t$ means evaluation at $X=X_{t}$ and $k=k_{t}=k^{n \ell}\left(X_{t}\right)$, and

$$
\Delta_{X, t}^{(1)}=\left.\frac{d}{d X} \Delta^{(1)}\left(k^{n \ell}(X), X\right)\right|_{X=X_{t}} .
$$

In the same fashion one obtains for the second harmonic

$$
\psi_{0}^{(2)}(X) \sim \hat{\Psi}_{0}^{(2)} e^{i 2 \theta_{0}(X)}\left(X-X_{t}\right)
$$

with, according to (110),

$$
\hat{\Psi}_{0}^{(2)}=\frac{N_{2, t}}{\Delta_{t}^{(2)}}\left(\hat{\Psi}_{0}^{(1)}\right)^{2} .
$$

In the bulk of the nonlinear region the harmonic spectrum is fully developed but towards the neutral stability boundary the higher order harmonics become slaved to the fundamental. Since the higher order harmonics decay faster than $\Psi^{(1)}$ as the neutral stability boundary is approached, the nonlinear solution is approximated by a purely sinusoidal wave of vanishing amplitude. Matching to a linear solution in the region $X<X_{t}$ therefore becomes possible.

\subsubsection{Diverging slow phase}

So far only the behavior of the amplitude as $X \downarrow X_{t}$ has been obtained. In this subsection the asymptotic behavior of the slow phase $\theta_{0}(X)$ at the neutral 
stability boundary of the nonlinear region is computed.

Let us write the solvability condition (58) as

$$
0=\frac{d \theta_{0}}{d X}\left\langle\Psi_{\theta}^{\dagger}, \mathcal{L}^{\prime} \Psi_{\theta}\right\rangle+\frac{1}{2} \frac{d k^{n \ell}}{d X}\left\langle\Psi_{\theta}^{\dagger}, \mathcal{L}^{\prime \prime} \Psi_{\theta}\right\rangle+\left\langle\Psi_{\theta}^{\dagger}, \mathcal{L}^{\prime}\left(\frac{d k^{n \ell}}{d X} \Psi_{k}+\Psi_{X}\right)\right\rangle
$$

According to (106) the nonlinear solution $\Psi$ admits the expansion

$$
\Psi\left(\theta+\theta_{0}(X) ; k^{n \ell}(X), X\right)=\sum_{n} \Psi^{(n)}\left(k^{n \ell}(X), X\right) e^{i n \theta_{0}(X)} e^{i n \theta}
$$

Thus

$$
\begin{aligned}
\mathcal{L}^{\prime}\left(\frac{d k^{n \ell}}{d X} \Psi_{k}+\Psi_{X}\right) & =\sum_{n} \mathcal{L}^{\prime}\left(i n k^{n \ell}(X) ; X\right) \frac{d}{d X}\left[\Psi^{(n)}(X)\right] e^{i n \theta_{0}(X)} e^{i n \theta} \\
\mathcal{L}^{\prime \prime} \Psi_{\theta} & =\sum_{n} i n \mathcal{L}^{\prime \prime}\left(i n k^{n \ell}(X) ; X\right) \Psi^{(n)}(X) e^{i n \theta_{0}(X)} e^{i n \theta} \\
\mathcal{L}^{\prime} \Psi_{\theta} & =\sum_{n} i n \mathcal{L}^{\prime}\left(i n k^{n \ell}(X) ; X\right) \Psi^{(n)}(X) e^{i n \theta_{0}(X)} e^{i n \theta}
\end{aligned}
$$

with

$$
\Psi^{(n)}(X) \equiv \Psi^{(n)}\left(k^{n \ell}(X), X\right) .
$$

According to $(109,114)$ the harmonic components exhibit the following behavior

$$
\Psi^{(n)}(X) \sim\left\{\begin{array}{ll}
\left(X-X_{t}\right)^{|n| / 2} \hat{\Psi}^{(n)} & \text { if } n \neq 0 \\
\left(X-X_{t}\right)^{2} \hat{\Psi}^{(0)} & \text { if } n=0
\end{array} \quad \text { as } \quad X \downarrow X_{t},\right.
$$

where $\hat{\Psi}^{(1)}$ and $\hat{\Psi}^{(2)}$ have explicitly been obtained in $(118,120)$. Thus the term $\mathcal{L}^{\prime}\left(k_{X}^{n \ell} \Psi_{k}+\Psi_{X}\right)$ diverges as $\left(X-X_{t}\right)^{-1 / 2}$ whereas the other terms scale as $\left(X-X_{t}\right)^{1 / 2}$. In order to evaluate the inner products involved in (121), the adjoint $\Psi_{\theta}^{\dagger}$ has to be explicitly determined. The term in $L^{\dagger}$ (57) associated with the nonlinear operator $\mathcal{N}$ reads

$$
\frac{\partial \mathcal{N}(X)[\Psi]}{\partial \psi}=2\left(X-X_{t}\right)^{1 / 2} N_{2, t}\left(\hat{\Psi}^{(1)} e^{i \theta_{0}(X)} e^{i \theta}+\text { c.c. }\right)+\mathcal{O}\left[X-X_{t}\right]
$$

whereas

$$
\begin{aligned}
\mathcal{L}\left(-k^{n \ell}(X) \partial_{\theta} ; X\right) & =\mathcal{L}\left(-k_{t} \partial_{\theta} ; X_{t}\right) \\
& +\left(X-X_{t}\right)\left(-k_{X, t}^{n \ell} \mathcal{L}^{\prime}\left(-k_{t} \partial_{\theta} ; X_{t}\right) \partial_{\theta}+\mathcal{L}_{X}\left(-k_{t} \partial_{\theta} ; X_{t}\right)\right) \\
& +\mathcal{O}\left[\left(X-X_{t}\right)^{2}\right] .
\end{aligned}
$$

Thus the adjoint operator (57) reads

$$
\begin{aligned}
L^{\dagger}= & -\omega_{g} \partial_{\theta}+\mathcal{L}\left(-k_{t} \partial_{\theta} ; X_{t}\right) \\
& +2\left(X-X_{t}\right)^{1 / 2} N_{2, t}\left(\hat{\Psi}^{(1)} e^{i \theta_{0}(X)} e^{i \theta}+\text { c.c. }\right)+\mathcal{O}\left[X-X_{t}\right] .
\end{aligned}
$$


A solution of $L^{\dagger} \phi=0$ sought under the form

$$
\phi=\sum_{n} i n\left(X-X_{t}\right)^{|n| / 2} \Phi^{(n)}(X) e^{i n \theta}
$$

leads to

$$
\begin{aligned}
0 & =\left(X-X_{t}\right)^{1 / 2}\left[\left(-i \omega_{g}+\mathcal{L}\left(-i k_{t} ; X_{t}\right)\right) i \Phi^{(1)}(X) e^{i \theta}+\text { c.c. }\right] \\
& +\left(X-X_{t}\right)\left[\left(-2 i \omega_{g}+\mathcal{L}\left(-2 i k_{t} ; X_{t}\right)\right) 2 i \Phi^{(2)}(X) e^{i 2 \theta}+\text { c.c. }\right] \\
& +\left(X-X_{t}\right) 2 N_{2, t}\left[\hat{\Psi}^{(1)} e^{i \theta_{0}(X)} e^{i \theta}+\text { c.c. }\right]\left[i \Phi^{(1)}(X) e^{i \theta}+\text { c.c. }\right] \\
& +\mathcal{O}\left[\left(X-X_{t}\right)^{3 / 2}\right] .
\end{aligned}
$$

Due to (103) the $\mathcal{O}\left[\left(X-X_{t}\right)^{1 / 2}\right]$ term identically vanishes. At order $\left(X-X_{t}\right)$, the equations

$$
\begin{aligned}
& 0=2 i N_{2, t} \hat{\Psi}^{(1)} e^{i \theta_{0}(X)} \Phi^{(-1)}(X)+\text { c.c. } \\
& 0=\left(2 \omega_{g}+i \mathcal{L}\left(-2 i k_{t}, X_{t}\right)\right) 2 \Phi^{(2)}(X)+2 N_{2}\left(X_{t}\right) \hat{\Psi}^{(1)} e^{i \theta_{0}(X)} i \Phi^{(1)}(X),
\end{aligned}
$$

yield

$$
\begin{aligned}
& \Phi^{(1)}(X) \propto \hat{\Psi}^{(1)} e^{i \theta_{0}(X)} \\
& \Phi^{(2)}(X) \propto \frac{i N_{2}\left(X_{t}\right)}{2 \omega_{g}+i \mathcal{L}\left(-2 i k_{t}, X_{t}\right)}\left(\hat{\Psi}^{(1)} e^{i \theta_{0}(X)}\right)^{2}
\end{aligned}
$$

with a real constant of proportionality. A simple calculation then leads to

$$
\begin{aligned}
\left\langle\Psi_{\theta}^{\dagger}, \mathcal{L}^{\prime} \Psi_{\theta}\right\rangle & =\left(X-X_{t}\right)\left[\mathcal{L}^{\prime}\left(i k_{t} ; X_{t}\right)+\text { c.c. }\right]\left|\hat{\Psi}^{(1)}\right|^{2}+\mathcal{O}\left[\left(X-X_{t}\right)^{2}\right], \\
\left\langle\Psi_{\theta}^{\dagger}, \mathcal{L}^{\prime \prime} \Psi_{\theta}\right\rangle & =\left(X-X_{t}\right)\left[\mathcal{L}^{\prime \prime}\left(i k_{t} ; X_{t}\right)+\text { c.c. }\right]\left|\hat{\Psi}^{(1)}\right|^{2}+\mathcal{O}\left[\left(X-X_{t}\right)^{2}\right], \\
\left\langle\Psi_{\theta}^{\dagger}, \mathcal{L}^{\prime}\left(k_{X}^{n \ell} \Psi_{k}+\Psi_{X}\right)\right\rangle & =-\frac{1}{2}\left[i \mathcal{L}^{\prime}\left(i k_{t} ; X_{t}\right)+\text { c.c. }\right]\left|\hat{\Psi}^{(1)}\right|^{2}+\mathcal{O}\left[X-X_{t}\right] .
\end{aligned}
$$

Upon substituting the preceding results into (121) the governing equation for the slow phase at the boundary of the nonlinear region becomes

$$
\frac{d \theta_{0}}{d X}=-\frac{1}{2} \frac{1}{X-X_{t}} \frac{\operatorname{Im} \mathcal{L}^{\prime}\left(i k_{t} ; X_{t}\right)}{\operatorname{Re} \mathcal{L}^{\prime}\left(i k_{t} ; X_{t}\right)}+\mathcal{O}\left[\left(X-X_{t}\right)^{0}\right]
$$

Hence, $\theta_{0}$ diverges logarithmically as

$$
\theta_{0}(X)=-\frac{1}{2} \frac{\operatorname{Im} \mathcal{L}^{\prime}\left(i k_{t} ; X_{t}\right)}{\operatorname{Re} \mathcal{L}^{\prime}\left(i k_{t} ; X_{t}\right)} \ln \left(X-X_{t}\right)+\mathrm{C}^{\text {st }}+\mathcal{O}\left[X-X_{t}\right],
$$

when $X \downarrow X_{t}$.

\subsubsection{Inner transition layer solution}

The asymptotic matching of a finite amplitude nonlinear wave train to an exponentially decaying linear solution takes place via an inner solution valid 
in a narrow transition layer at $X_{t}$ between the nonlinear and linear regions.

Define an inner variable $\tilde{X}$ in the neighborhood of $X_{t}$ by $X=X_{t}+\epsilon^{1 / 2} \tilde{X}$. The outer solution obtained in the previous section is rewritten in terms of this inner variable. The fast phase $\theta$ reads

$$
\theta \equiv \frac{1}{\epsilon} \int^{X_{t}+\epsilon^{1 / 2} \tilde{X}} k^{n \ell}(u) d u-\omega_{g} t=\left(k_{t} x-\omega_{g} t\right)+\frac{1}{2} k_{X, t}^{n \ell} \tilde{X}^{2}+\mathcal{O}\left(\epsilon^{1 / 2}\right)
$$

whereas the slow phase $\theta_{0}$ of eq.(122) is expanded as

$$
\theta_{0} \sim-\frac{1}{2} \frac{\operatorname{Im} \mathcal{L}^{\prime}\left(i k_{t} ; X_{t}\right)}{\operatorname{Re} \mathcal{L}^{\prime}\left(i k_{t} ; X_{t}\right)} \ln \tilde{X}+\mathrm{C}^{\mathrm{st}}
$$

Thus, as $X \downarrow X_{t}$, keeping only the leading order terms in the harmonics $|n| \leq 2$ of the outer solution given by (115) yields the following expansion

$$
\begin{aligned}
\psi & \sim \epsilon^{1 / 4} \tilde{X}^{1 / 2}\left[\hat{\Psi}_{0}^{(1)} e^{i\left(\theta+\theta_{0}\right)}+\text { c.c. }\right] \\
& +\epsilon^{1 / 2} \tilde{X}\left(\frac{i N_{2, t}}{\Delta_{t}^{(2)}}\left[\left(\hat{\Psi}_{0}^{(1)}\right)^{2} e^{2 i\left(\theta+\theta_{0}\right)}+\text { c.c. }\right]+\frac{i N_{2, t}}{\Delta_{t}^{(0)}} 2\left|\hat{\Psi}_{0}^{(1)}\right|^{2}\right)+\mathcal{O}\left(\epsilon^{3 / 4}\right) .
\end{aligned}
$$

This behavior of the outer solution in terms of the inner variable $\tilde{X}$ suggests to expand the harmonic components of the inner solution as

$$
\psi \sim \sum_{n} \epsilon^{n / 4}\left(\tilde{\psi}_{0}^{(n)}(x, \tilde{X})+\epsilon^{1 / 2} \tilde{\psi}_{1 / 2}^{(n)}(x, \tilde{X})+\ldots\right) e^{-i n \omega_{g} t}
$$

with $\tilde{\psi}_{0}^{(0)}=0$ since $\psi^{(0)}=\mathcal{O}\left(\left|\psi^{(1)}\right|^{2}\right)$. Each component of the inner expansion has to be matched for $\tilde{X} \rightarrow+\infty$ with the corresponding component of the outer expansion. Due to the presence of slow and fast spatial scales $\tilde{X}$ and $x$, the spatial derivative in the governing equation (1) now reads $\partial_{x}+\epsilon^{1 / 2} \partial_{\tilde{X}}$. Thus the following equations are obtained for each harmonic component

$$
\begin{aligned}
0= & \left(i n \omega_{g}+\mathcal{L}\left(\partial_{x}+\epsilon^{1 / 2} \partial_{\tilde{X}} ; X_{t}+\epsilon^{1 / 2} \tilde{X}\right)\right)\left[\tilde{\psi}_{0}^{(n)}+\epsilon^{1 / 2} \tilde{\psi}_{1 / 2}^{(n)}+\ldots\right] \\
& +\sum_{r=2}^{\infty} N_{r}\left(X_{t}+\epsilon^{1 / 2} \tilde{X}\right) \sum_{n_{1}+\ldots+n_{r}=n}\left(\tilde{\psi}_{0}^{\left(n_{1}\right)}+\ldots\right) \cdots\left(\tilde{\psi}_{0}^{\left(n_{r}\right)}+\ldots\right) .
\end{aligned}
$$

For $n=1$ the leading order problem in $\epsilon$ reads

$$
\omega_{g} \tilde{\psi}_{0}^{(1)}=i \mathcal{L}\left(\partial_{x} ; X_{t}\right)\left[\widetilde{\psi}_{0}^{(1)}\right]
$$

This linear differential equation in $x$ admits solutions of the form $A(\tilde{X}) e^{i k x}$ where the a priori possible complex wave numbers $k$ satisfy the linear dispersion relation (20): $\omega_{g}=\Omega^{\ell}\left(k, X_{t}\right)$. However, the slowly (algebraically) decaying 
outer nonlinear solution is oscillating on the fast scale as $e^{i k_{t} x}$ with $k_{t}$ defined by (103). Hence matching of outer and inner solutions ensures that $\widetilde{\psi}_{0}^{(1)}$ contains the single Fourier mode

$$
\tilde{\psi}_{0}^{(1)}=\tilde{A}_{0}^{(1)}(\tilde{X}) e^{i k_{t} x}
$$

with a slowly varying amplitude $\tilde{A}_{0}^{(1)}(\tilde{X})$.

For $n=2$ the leading order problem in $\epsilon$ of (127) reads

$$
2 \omega_{g} \tilde{\psi}_{0}^{(2)}-i \mathcal{L}\left(\partial_{x} ; X_{t}\right)\left[\tilde{\psi}_{0}^{(2)}\right]=i N_{2, t} \tilde{\psi}_{0}^{(1)} \tilde{\psi}_{0}^{(1)}
$$

Using (128) yields the particular solution

$$
\tilde{\psi}_{0}^{(2)}=\frac{i N_{2, t}}{\Delta_{t}^{(2)}}\left[\tilde{A}_{0}^{(1)}(\tilde{X})\right]^{2} e^{2 i k_{t} x} .
$$

The homogenous differential equation associated with (129) admits solutions of the form $e^{i k_{2} x}$ for wave numbers $k_{2}$ satisfying $2 \omega_{g}=\Omega^{\ell}\left(k_{2}, X_{t}\right)$. Again none of these complex wave numbers $k_{2}$ satisfy the matching conditions, thus only the particular solution (130) needs to be retained.

For $n=0$, the equation

$$
0=\mathcal{L}\left(\partial_{x} ; X_{t}\right)\left[\tilde{\psi}_{1 / 2}^{(0)}\right]+2 N_{2, t}\left|\tilde{\psi}_{0}^{(1)}\right|^{2}
$$

yields

$$
\tilde{\psi}_{1 / 2}^{(0)}=\frac{2 i N_{2, t}}{\Delta_{t}^{(0)}}\left|\tilde{A}_{0}^{(1)}(\tilde{X})\right|^{2} .
$$

In eq.(127), the linear operator expands as

$$
\begin{aligned}
\mathcal{L}\left(\partial_{x}+\epsilon^{1 / 2} \partial_{\tilde{X}} ; X_{t}+\epsilon^{1 / 2} \tilde{X}\right)= & \mathcal{L}\left(\partial_{x} ; X_{t}\right) \\
& +\epsilon^{1 / 2}\left[\mathcal{L}^{\prime}\left(\partial_{x} ; X_{t}\right) \partial_{\tilde{X}}+\tilde{X} \mathcal{L}_{X}\left(\partial_{x} ; X_{t}\right)\right]+\mathcal{O}(\epsilon)
\end{aligned}
$$

Thus for $n=1$ the order $\epsilon^{1 / 2}$ terms of (127) read

$$
\begin{aligned}
0= & i \omega_{g} \tilde{\psi}_{1 / 2}^{(1)}+\mathcal{L}\left(\partial_{x} ; X_{t}\right)\left[\tilde{\psi}_{1 / 2}^{(1)}\right]+\left[\mathcal{L}^{\prime}\left(\partial_{x} ; X_{t}\right) \partial_{\tilde{X}}+\tilde{X} \mathcal{L}_{X}\left(\partial_{x} ; X_{t}\right)\right] \tilde{\psi}_{0}^{(1)} \\
& +3 N_{3, t}\left|\tilde{\psi}_{0}^{(1)}\right|^{2} \tilde{\psi}_{0}^{(1)}+2 N_{2, t}\left[\tilde{\psi}_{0}^{(2)} \tilde{\psi}_{0}^{(-1)}+\tilde{\psi}_{1 / 2}^{(0)} \tilde{\psi}_{0}^{(1)}\right]
\end{aligned}
$$

and at this order there are no derivatives of the nonlinear term with respect to $X$. This inhomogenous differential equation in $x$ admits solutions for $\widetilde{\psi}_{1 / 2}^{(1)}$ only if the resonant terms in $e^{i k_{t} x}$ of the forcing terms vanish. Upon using $(128,130,131)$ in $(132)$, this leads to the solvability condition 


$$
\begin{aligned}
0= & \mathcal{L}^{\prime}\left(i k_{t} ; X_{t}\right) \frac{d \tilde{A}_{0}^{(1)}}{d \tilde{X}}+\mathcal{L}_{X}\left(i k_{t} ; X_{t}\right) \tilde{X} \tilde{A}_{0}^{(1)}(\tilde{X}) \\
& +\left(3 N_{3, t}+2 i\left(N_{2, t}\right)^{2}\left(\frac{1}{\Delta_{t}^{(2)}}+\frac{2}{\Delta_{t}^{(0)}}\right)\right)\left|\tilde{A}_{0}^{(1)}(\tilde{X})\right|^{2} \tilde{A}_{0}^{(1)}(\tilde{X})
\end{aligned}
$$

This amplitude equation for $\tilde{A}_{0}^{(1)}(\tilde{X})$ is of the form

$$
\frac{d \tilde{A}_{0}^{(1)}}{d \tilde{X}}+\left(\left(a_{r}+i a_{i}\right) \tilde{X}+\left(b_{r}+i b_{i}\right)\left|\tilde{A}_{0}^{(1)}(\tilde{X})\right|^{2}\right) \tilde{A}_{0}^{(1)}(\tilde{X})=0
$$

and its solutions are

$$
\frac{\tilde{A}_{0}^{(1)}(\tilde{X})}{\tilde{A}_{0}^{(1)}(0)}=\frac{\exp \left[-\frac{a_{r}+i a_{i}}{2} \tilde{X}^{2}-\frac{i}{2} \frac{b_{i}}{b_{r}} \ln \left(1+2 b_{r}\left|\tilde{A}_{0}^{(1)}(0)\right|^{2} \int_{0}^{\tilde{X}} e^{-a_{r} u^{2}} d u\right)\right]}{\sqrt{1+2 b_{r}\left|\tilde{A}_{0}^{(1)}(0)\right|^{2} \int_{0}^{\tilde{X}} e^{-a_{r} u^{2}} d u}}
$$

where $\tilde{A}_{0}^{(1)}(0)$ is the integration constant. Matching of the inner solution $\tilde{\psi}_{0}^{(1)}$ to the outer solution $\psi_{0}^{(1)}$ of (117) requires that $\left|\tilde{A}_{0}^{(1)}(\tilde{X})\right| \sim \sqrt{\tilde{X}}$ as $\tilde{X} \rightarrow+\infty$. This implies that

$$
1+2 b_{r}\left|\tilde{A}_{0}^{(1)}(0)\right|^{2} \int_{0}^{+\infty} e^{-a_{r} u^{2}} d u=0, \quad \text { i.e., } \quad\left|\tilde{A}_{0}^{(1)}(0)\right|^{2}=\frac{-1}{b_{r}} \sqrt{\frac{a_{r}}{\pi}} .
$$

With this value for $\left|\tilde{A}_{0}^{(1)}(0)\right|$ the inner solution (135) admits for $\tilde{X} \rightarrow+\infty$ the asymptotic expansion

$$
\tilde{A}_{0}^{(1)}(\tilde{X}) \sim \sqrt{-\frac{a_{r}}{b_{r}} \tilde{X}} \exp \frac{i}{2}\left[\left(\frac{b_{i}}{b_{r}} a_{r}-a_{i}\right) \tilde{X}^{2}+\frac{b_{i}}{b_{r}} \ln \tilde{X}+\mathrm{C}^{\mathrm{st}}\right] .
$$

Comparison of (133) and (134) yields

$$
\begin{aligned}
a_{r}+i a_{i} & =\frac{\mathcal{L}_{X}\left(i k_{t} ; X_{t}\right)}{\mathcal{L}^{\prime}\left(i k_{t} ; X_{t}\right)}=-i k_{X, t}^{\ell}, \\
b_{r}+i b_{i} & =\left|\hat{\Psi}_{0}^{(1)}\right|^{-2} \frac{\Delta_{X, t}^{(1)}}{i \mathcal{L}^{\prime}\left(i k_{t} ; X_{t}\right)}=-\left|\hat{\Psi}_{0}^{(1)}\right|^{-2}\left(i k_{X, t}^{n \ell}+\frac{\mathcal{L}_{X}\left(i k_{t} ; X_{t}\right)}{\mathcal{L}^{\prime}\left(i k_{t} ; X_{t}\right)}\right)
\end{aligned}
$$

with $\left|\hat{\Psi}_{0}^{(1)}\right|^{2}$ obtained in (118). Thus

$$
\begin{aligned}
-\frac{a_{r}}{b_{r}} & =\left|\hat{\Psi}_{0}^{(1)}\right|^{2}, \\
\frac{b_{i}}{b_{r}} a_{r}-a_{i} & =k_{X, t}^{n \ell}, \\
-\frac{b_{i}}{b_{r}} & =\frac{\operatorname{Im} \mathcal{L}^{\prime}\left(i k_{t}, X_{t}\right)}{\operatorname{Re} \mathcal{L}^{\prime}\left(i k_{t}, X_{t}\right)} .
\end{aligned}
$$


This completes the proof that the first harmonic of the outer nonlinear solution given by eq.(125) completely matches the first harmonic of the inner weakly nonlinear solution $\epsilon^{1 / 4} \tilde{A}_{0}^{(1)}(\tilde{X}) e^{i\left(k_{t} x-\omega_{g} t\right)}$, where $\tilde{A}_{0}^{(1)}(\tilde{X})$ is given by (135).

\subsubsection{Matching to the outer linear solution}

As $X \uparrow X_{t}$, the outer linear WKBJ approximation (41) reads, at leading order,

$$
\psi \sim A_{0}^{\ell}\left(X_{t}\right) e^{i\left(k_{t} x-\omega_{g} t\right)} \exp \left(\frac{i}{2} k_{X, t}^{\ell} \tilde{X}^{2}\right)+\text { c.c. }
$$

when expressed in terms of the inner variable $\tilde{X}$. Using (135), the asymptotic behavior of the inner solution as $\tilde{X} \rightarrow-\infty$ yields

$$
\begin{aligned}
\psi & \sim \epsilon^{1 / 4} \tilde{A}_{0}^{(1)}(\tilde{X}) e^{i\left(k_{t} x-\omega_{g} t\right)}+\text { c.c. } \\
& \sim \epsilon^{1 / 4} \frac{\tilde{A}_{0}^{(1)}(0)}{\sqrt{2}} e^{i\left(k_{t} x-\omega_{g} t\right)} \exp \left(-\frac{a_{r}+i a_{i}}{2} \tilde{X}^{2}-\frac{i}{2} \frac{b_{i}}{b_{r}} \ln 2\right)+\text { c.c. }
\end{aligned}
$$

Since $a_{r}+i a_{i}=-i k_{X, t}^{\ell}$, both expansions (137) and (138) asymptotically match with

$$
A_{0}^{\ell}\left(X_{t}\right)=\epsilon^{1 / 4} \frac{\tilde{A}_{0}^{(1)}(0)}{\sqrt{2}} e^{-i \frac{b_{i} \ln 2}{2 b_{r}}} .
$$

Thus, at leading order, the weakly nonlinear inner expansion (138) exactly matches the outer linear WKBJ approximation (41). In the inner transition layer the slaved higher order harmonics scale as $\epsilon^{|n| / 4}$. In the outer linear region, each harmonic component matches the corresponding exponentially slaved harmonic and decays as

$$
\epsilon^{|n| / 4} \exp -\frac{|n|}{\epsilon} \int^{X} k_{i}^{\ell}(u) d u .
$$

The width of the transition layer at $X_{t}$ is of order $\epsilon^{1 / 2}$ measured in terms of $X$. The intermediate scaling between the fast length scale $x$ and the slow spatial variation $X=\epsilon x$ allows the asymptotic analysis of the crossover between linear and nonlinear regions. Even in the narrow transition layer the solution exhibits slowly modulated fast oscillations; the transition layer contains $\mathcal{O}\left(\epsilon^{-1 / 2}\right)$ wave lengths.

\section{Conclusions}

It has been demonstrated that a wide class of one-dimensional nonlinear evolution equations with spatially varying coefficients may support two types of 
fully nonlinear self-sustained global modes in a doubly infinite domain: Steep global modes are triggered by the presence of a sharp stationary front located at the upstream transition point between local convective and absolute instability. This front acts as a source and imposes its real absolute frequency to the entire medium. Soft global modes are due to the presence of a saddle point of the local nonlinear dispersion relation which again acts as a source and imposes its frequency to the entire medium, as given by saddle point conditions.

A necessary condition for the occurrence of either of these modes is the existence of a region of local absolute instability. Recall that linearly unstable global modes given by a complex saddle point of the local linear dispersion relation $[7,25,30]$ also require a range of local absolute instability. But, this range must in general be of finite extent whereas nonlinear global modes exist, however small the AU domain. The relationship between linear global instability and the existence of fully nonlinear global modes is non-trivial: In the generic case, nonlinear global instability does not coincide with linear global instability. The nature of the various global bifurcation scenarii constitute the major result of the present investigation. Steep global modes occur right at local absolute instability onset below the linear global instability threshold, via a saddle node bifurcation, while the medium is still linearly globally stable.

Soft global modes generically do not appear at local absolute instability onset but only for a sufficiently large domain of local absolute instability. Furthermore, they are more likely to be observed in systems with weak advection. It is essential to note that steep and soft global modes are mutually exclusive, as dictated by the relative magnitude of their respective frequencies. The existence and selection of either kind of global modes has been confirmed by direct numerical simulations of the CGL equation with varying coefficients for small but finite values of $\epsilon$. As a word of caution, it should be stated that soft global modes are likely to be more fragile than their steep counterparts. They may become unstable whenever the region of local absolute instability protrudes beyond the central nonlinear regions, in the tails of the extended wavepacket. This lack of robustness is all the more acute as the WKBJ limit $\epsilon=0$ is approached.

In several physical systems [36,1] linear and nonlinear global instability have been found to occur at the same value of the global control parameter. This peculiar feature takes place in situations where the entire spatial dependence is accounted for in a single real spatially varying parameter, say $R(X)$, which displays an extremum at a real position $X^{\max }$ such that $d R\left(X^{\max }\right) / d X=0$. Under these circumstances, the local linear dispersion relation is necessarily of the form $\Omega^{\ell}(k, X)=\Omega_{\star}^{\ell}[k, R(X)]$ and the local absolute frequency is readily obtained as $\omega_{0}(X)=\omega_{0 \star}[R(X)]$. The real station $X^{\max }$ is then simultaneously associated with a maximum of $\omega_{0, i}(X)$ and with a saddle point $X_{s}^{\ell}$ of $\omega_{0}(X)$. 
The analytical structure underlying the spatial distribution of steep and soft global modes has been systematically derived in the WKBJ approximation $\epsilon \ll 1$. It has been shown that for a wide class of one-dimensional evolution equations the various inner layers and outer regions may be matched together to arrive at a consistent description valid over the entire spatial domain. In particular, higher-order frequency corrections have been obtained.

It should be emphasized that the different transition scenarii depend on the precise form of the linear and nonlinear dispersion relations. Due to the number of parameters required to specify the spatial variations of the medium, a comprehensive survey of all possible configurations has not been attempted.

This study has been undertaken in order to understand the nature of synchronized structures in real slowly varying open shear flows. In the latter framework, the local linear dispersion relation is obtained from the Rayleigh or the Orr-Sommerfeld equation, whereas the local nonlinear dispersion relation requires the computation of finite amplitude structures in a streamwise periodic interval. In this context, steep global modes may be constructed as reported elsewhere [35].

\section{Ackowledgements}

The authors have benefitted from many enlightening and fruitful discussions with Arnaud Couairon.

\section{References}

[1] A.P. Bassom, K.M. Kuzanyan, and A.M. Soward, A nonlinear dynamo wave riding on a spatially varying background, Proc. R. Soc. Lond. A, 455, 14431481 (1999).

[2] C.M. Bender and S.A. Orszag, Advanced mathematical methods for scientists and engineers (McGraw-Hill, New York, 1978).

[3] A. Bers, Space-time evolution of plasma instabilities - absolute and convective, in Handbook of plasma physics, M.N. Rosenbluth and R.Z. Sagdeev Eds., North-Holland, Amsterdam, 451-517, (1983).

[4] R.J. Briggs, Electron-stream interaction with plasmas, (M.I.T. Press, Cambridge, Mass., 1964).

[5] P. Büchel, M. Lücke, D. Roth and R. Schmitz, Pattern selection in the absolutely unstable regime as a nonlinear eigenvalue problem: Taylor vortices in axial flow. Phys. Rev. E 53, 4764-4777 (1996). 
[6] J.-M. Chomaz, P. Huerre and L.G. Redekopp, The effect of nonlinearity and forcing on global modes, in New Trends in nonlinear dynamics and patternforming phenomena, P. Coullet and P. Huerre, eds., (Plenum Press, New York, 1990), 259-274.

[7] J.-M. Chomaz, P. Huerre and L.G. Redekopp, A frequency selection criterion in spatially developing flows, Stud. Appl. Math. 84, 119-144 (1991).

[8] J.-M. Chomaz, Absolute and convective instabilities in nonlinear systems, Phys. Rev. Lett. 69, 1931-1934 (1992).

[9] J.-M. Chomaz and A. Couairon, Against the wind, Phys. Fluids. 11, 2977-2983 (1999).

[10] A. Couairon and J.-M. Chomaz, Global instability in fully nonlinear systems, Phys. Rev. Lett. 77, 4015-4018 (1996).

[11] A. Couairon, Modes globaux fortement non linéaires dans les écoulements ouverts, $\mathrm{PhD}$ thesis, École polytechnique (1997).

[12] A. Couairon and J.-M. Chomaz, Absolute and convective instabilities, front velocities and global modes in nonlinear systems, Physica D 108, 236-276 (1997).

[13] A. Couairon and J.-M. Chomaz, Pattern selection in the presence of cross flow, Phys. Rev. Lett. 79, 2666-2669 (1997).

[14] A. Couairon and J.-M. Chomaz, Fully nonlinear global modes in slowly varying flows. Phys. Fluids 11, 3688-3703 (1999).

[15] M.C. Cross and P.C. Hohenberg, Pattern formation outside of equilibrium, Rev. Mod. Phys. 65, 851-1112 (1993).

[16] G.T. Dee and J.S. Langer, Propagating pattern selection, Phys. Rev. Lett. 50, 383-386 (1983).

[17] T.M. DelSole and B.F. Farrell, Nonlinear equilibrium of localized instabilities on a baroclinic jet, J. Atmos. Sci. 51, 2270-2284 (1994).

[18] K. Hannemann and H. Oertel Jr, Numerical simulation of the absolutely and convectively unstable wake, J. Fluid Mech. 199, 55-88 (1989).

[19] D.A. Hammond and L.G. Redekopp, Global dynamics of symmetric and asymmetric wakes, J. Fluid Mech. 331, 231-260 (1997).

[20] P. Huerre and P.A. Monkewitz, Local and global instabilities in spatially developing flows, Ann. Rev. Fluid Mech. 22, 473-537 (1990).

[21] P. Huerre and M. Rossi, Hydrodynamic instabilities in open flows, in Hydrodynamics and nonlinear instabilities, (eds. C. Godrèche and P. Manneville), Cambridge University Press, 81-294 (1998).

[22] P. Huerre, Open shear flow instabilities, to appear in Developments in Fluid Mechanics: A Collection for the Millenium (eds. G.K. Batchelor, H.K. Moffatt and M.G. Worster), Cambridge University Press (2000). 
[23] R.E. Hunt and D.G. Crighton, Instability of flows in spatially developing media, Proc. R. Soc. Lond. A 435, 109-128 (1991).

[24] S. Le Dizès, P. Huerre, J.-M. Chomaz and P.A. Monkewitz, Nonlinear stability analysis of slowly-diverging flows: limitations of the weakly nonlinear approach, Proc. IUTAM Symp. on Bluff-Body Wakes, Dynamics and Instabilities, H. Eckelmann, J.M.R. Graham, P. Huerre and P.A. Monkewitz Eds., Springer, Berlin, 147-152 (1993).

[25] S. Le Dizès, P. Huerre, J.-M. Chomaz and P.A. Monkewitz, Linear global modes in spatially developing media, Phil. Trans. R. Soc. Lond. A 354, 169-212 (1996).

[26] P. Manneville, Structures dissipatives, chaos et turbulence (Aléa, Saclay, 1991).

[27] C. Mathis, M. Provansal and L. Boyer, The Bénard-von Karman instability: an experimental study near the threshold, J. Phys. Paris Lett. 45, L83-L491 (1984).

[28] N. Meunier, M.R.E. Proctor, D.D. Sokoloff, A.M. Soward, and S.M. Tobias, Asymptotic properties of a nonlinear $\alpha \omega$-dynamo wave: period, amplitude and latitude dependence, Geophys. Astrophys. Fluid Dynamics 86, 249-285 (1997).

[29] P.A. Monkewitz, D.W. Bechert, B. Barsikowr and B. Lehmann, Self-excited oscillations and mixing in heated round jets, J. Fluid Mech. 213, 611-639 (1990).

[30] P.A. Monkewitz, P. Huerre and J.M. Chomaz, Global linear stability analysis of weakly non-parallel shear flows, J. Fluid Mech. 251, 1-20 (1993).

[31] H.W. Müller, M. Lücke and M. Kamps, Transversal convection patterns in horizontal shear flow. Phys. Rev. A 45, 3714-3726 (1992).

[32] A.C. Newell, T. Passot, J. Lega, Order parameter equations for patterns, Ann. Rev. Fluid Mech. 25, 399-453 (1993).

[33] B. Pier and P. Huerre, Fully nonlinear global modes in spatially developing media, Physica D 97, 206-222 (1996).

[34] B. Pier, P. Huerre, J.-M. Chomaz and A. Couairon, Steep nonlinear global modes in spatially developing media. Phys. Fluids 10, 2433-2435 (1998).

[35] B. Pier and P. Huerre, Nonlinear self-sustained structures and fronts in spatially developing wake flows. in preparation.

[36] R.T. Pierrehumbert, Local and global baroclinic instability of zonally varying flow, J. Atmos. Sc. 41, 2141-2162 (1984).

[37] M. Provansal, C. Mathis and L. Boyer, Bénard-von Kármán instability: transient and forced regimes, J. Fluid Mech. 182, 1-22 (1987).

[38] W. van Saarloos, Dynamical velocity selection: marginal stability, Phys. Rev. Lett. 58, 2571-2574 (1987). 
[39] W. van Saarloos, Front propagation into unstable states: marginal stability as a dynamical mechanism for velocity selection, Phys. Rev. A 37, 211-229 (1988).

[40] W. van Saarloos, Front propagation into unstable states: II. Linear versus nonlinear marginal stability and rate of convergence Phys. Rev. A 39, 63676390 (1989).

[41] W. van Saarloos and P.C. Hohenberg, Fronts, pulses, sources and sinks in generalized complex Ginzburg-Landau equations, Physica D 56, 303-367 (1992).

[42] A.M. Soward and C.A. Jones, The linear stability of the flow in the narrow gap between two concentric rotating spheres, Q. Jl. Mech. Appl. Math. 36, 19-42 (1983).

[43] K.R. Sreenivasan, S. Raghu and D. Kyle, Absolute instability in variable density round jets, Exp. Fluids 7, 309-317 (1989).

[44] K.R. Sreenivasan, P.J. Strykowski and D.J. Olinger, On the Hopf bifurcation and the Landau-Stuart constants associated with vortex "shedding" behind circular cylinders, unpublished (1990).

[45] P.J. Strykowski and D.L. Niccum, The stability of countercurrent mixing layers in circular jets, J. Fluid Mech. 227, 309-343 (1991).

[46] S. Tobias, M.R.E. Proctor and E. Knobloch, Convective and absolute instabilities of fluid flows in finite geometry, Physica D 113, 43-72 (1998).

[47] W. Wasow, Linear turning point theory (Springer, New York, 1985).

[48] B.M. Woodley and N. Peake, Global linear stability analysis of thin aerofoil wakes, J. Fluid. Mech. 339, 239-260 (1997). 